\title{
The Evolution of Temperature and Bolometric Luminosity in Type-II Supernovae
}

\author{
T. Faran, ${ }^{1 \star}$ E. Nakar, ${ }^{2}$ and D. Poznanski ${ }^{2}$ \\ ${ }^{1}$ Racah Institute of Physics, The Hebrew University of Jerusalem, Jerusalem 91904, Israel \\ ${ }^{2}$ School of Physics and Astronomy, Tel-Aviv University, Tel Aviv 69978, Israel.
}

Accepted XXX. Received YYY; in original form ZZZ

\begin{abstract}
In this work we present a uniform analysis of the temperature evolution and bolometric luminosity of a sample of 29 type-II supernovae ( $\mathrm{SNe}$ ), by fitting a black body model to their multi-band photometry. Our sample includes only SNe with high quality multiband data and relatively well sampled time coverage. Most of the SNe in our sample were detected less than a week after explosion so their light curves cover the evolution both before and after recombination starts playing a role. We use this sample to study the signature of hydrogen recombination, which is expected to appear once the observed temperature drops to $\approx 7,000 \mathrm{~K}$. Theory predicts that before recombination starts affecting the light curve, both the luminosity and the temperature should drop relatively fast, following a power-law in time. Once the recombination front reaches inner parts of the outflow, it sets the observed temperature to be nearly constant, and slows the decline of the luminosity (or even leads to a re-brightening). We compare our data to analytic studies and find strong evidence for the signature of recombination. We also find that the onset of the optical plateau in a given filter, is effectively the time at which the black body peak reaches the central wavelength of the filter, as it cools, and it does not correspond to the time at which recombination starts affecting the emission.
\end{abstract}

\section{INTRODUCTION}

Type II supernovae (SNe) are defined by the prominent hydrogen lines in their spectra. They are believed to originate from the collapse of an iron core of massive stars $\left(\gtrsim 8 M_{\odot}\right)$ that retain their hydrogen envelope. The most common subtype, comprising $\sim 70$ percent of all type II SNe, is characterized by a phase of of a roughly constant magnitude in the optical bands, hence their name type II-Plateau (II-P). This plateau phase typically starts $1-2$ weeks after the explosion and lasts for $\sim 100 \mathrm{~d}$. Pre-explosion images have revealed that the progenitors of this class are red supergiants, in the mass range of $\left(7-16 M_{\odot}\right)$ (Smartt 2015; for individual progenitor detections see e.g. Van Dyk et al. 2003a, Van Dyk et al. 2003b, Van Dyk et al. 2012). Type II-Linear (II-L) SNe constitutes another subclass of type II SNe (e.g., Patat et al. 1994; Arcavi et al. 2012; Faran et al. 2014a,b). They are spectroscopically very similar to type II-P events (Faran et al. 2014b, see), but their light curves are declining in all bands. In both types (II-P and II-L) there is typically a sharp drop in the luminosity after $\sim 100 \mathrm{~d}$ and the luminosity starts to follow roughly the exponential decay expected from emission powered by the decay of ${ }^{56} \mathrm{Ni}$. The distinction between these two classes is not well defined, and studies have used different definitions for II-L SNe. However, several recent works have shown that there exists a continuum of decline rates between slow declining and fast declining $\mathrm{SNe}$ (Ander- son et al. 2014; Faran et al. 2014a), which suggests that a separation into two different classes may be artificial. Other type II sub-classes will not be discussed here. In this paper we focus on the light curves of type II-P and type II-L SNe, without making the distinction between the two types, and refer to them in short as type II SNe. Our goal here is to perform a uniform analysis of the bolometric luminosity and temperature evolution of a large sample of type II SNe and to compare our findings to theoretical models, focusing on the transition to the plateau, which takes place during the first two weeks.

There are several dozen type II SNe with detailed multiwavelength observations. These are typically presented and analyzed individually (e.g., Leonard et al. 2002b; Maguire et al. 2010; Pastorello et al. 2009; Inserra et al. 2011; Takáts et al. 2014, 2015; Fraser et al. 2011; Tomasella et al. 2013; Dall'Ora et al. 2014; Barbarino et al. 2015; Valenti et al. 2015). There are only a few studies that analyze the bolometric properties and temperatures of a sample of type-II SNe. Bersten \& Hamuy (2009) extracted bolometric light curves and effective temperature evolution for 33 SNe II-P, using calibrations for bolometric corrections from 3 well observed SNe. Valenti et al. (2016) derived the effective temperature from black body fits to photometric data of 30 type-II SNe, and calculated pseudo-bolometric light curves by integration over the optical bands. Lusk (2016) provides bolometric light curves and temperatures for 5 peculiar type II-P SNe that 
originated from blue supergiants, by integrating over the observed photometry and correcting for the missing flux in the UV.

The theoretical interpretation of type II light curves is that the emission until the end of the plateau is dominated by the cooling emission, i.e., the leakage of radiation energy that was deposited in the envelope by the shock that unbinds it (Falk \& Arnett 1977). Energy deposited by the decay of ${ }^{56}$ Ni may contribute to this phase (e.g., Falk \& Arnett 1977; Young 2004; Utrobin 2007), but this contribution is found to be subdominant (Nakar et al. 2016). The end of the plateau marks the release of all the internal energy deposited by the shock in the envelope. At later times the SN enters its nebular phase and the entire luminosity is driven by the decay of ${ }^{56} \mathrm{Ni}$.

During the early stages of the light curve ( $\sim 1-3$ weeks) the leakage of radiation is facilitated mostly by the drop in the optical depth of the outflow due to its expansion (Arnett 1980). Models predict that during this phase both the temperature and the bolometric luminosity drop roughly as power-laws in time (Nakar \& Sari 2010; Rabinak \& Waxman 2011; Shussman et al. 2016a). Once the observed temperature drop to $\approx 7000 \mathrm{~K}$, hydrogen recombination becomes important and a recombination front starts moving from the outside towards inner parts of the ejecta. During this phase, recombination, rather then expansion, is the main driver of the drop in the optical depth of the outflow. As a result the observed temperature remains almost constant while the luminosity starts dropping much more slowly or even rises.

In this work we derive the temperatures and bolometric evolution of 29 type-II SNe with high quality multi-band light curves, by fitting a black body spectrum to their spectral energy distribution (SEDs). As will be discussed extensively below, these are far from trivial. SNe II are not blackbodies, and at different times several effects lead to systematic offsets from a pure black body in various bands. Nevertheless, guided by the data and theoretical insight, we derive the underlying black body properties. We compare the temporal evolution of the temperature and luminosity to theoretical predictions, paying special attention to signs of the recombination processes in the envelope. In Section 2 we describe the contents of our SN sample and the data, in Section 3 we explain how the black body fits to the data are done. Section A describes the results of the fitting and the possible effect of extinction on the results, and Section 5 presents a comparison of our results to theoretical expectations. We summarize our results in Section. 6.

\section{THE SAMPLE}

We construct from the literature a sample of 29 type-II SNe with good temporal coverage and multi-band photometry. The sample mostly relies on the SNe collected in Pejcha \& Prieto (2015), Faran et al. (2014a) and (Faran et al. 2014b). The $\mathrm{SNe}$ in the sample were required to have sufficiently early data (starting less than 20 days after the explosion) and a well sampled light curves so the early behavior could be compared to the late behavior and to theoretical models. Some objects did not enter the sample despite having well sampled photometric curves, because their data was not good enough to produce good quality temperature and luminosity curves. Ten of the objects have Swift UV observations, 10 have JHK data, and 7 objects have both JHK and UV. The photometric data were corrected for galactic extinction according to Cardelli et al. (1989), but not for host galactic extinction, since there is no method that can provide an accurate estimate for $E(B-V)_{\text {host }}$ (see for example the discussion in Faran et al. 2014a). We note however, that Faran et al. (2014a) found that $E(B-V)_{h o s t}$ is typically small, of order 0.1 for nearby SNe. The explosion day is set as the mid-point between the first detection and the last nondetection of the $\mathrm{SN}$, and the uncertainty is conservatively set as half the difference. Distance measurements were collected from $\mathrm{NED}^{1}$ and averaged, using only distances based on the Tully-Fisher method, Cepheids, and SNe Ia. All of the objects are at low redshift with $\mathrm{z}<0.03$. The SN properties and their references are summarized in Table. 1.

\section{BLACK BODY FITTING}

We calculate the temperature and bolometric luminosity of the SNe at each epoch by fitting a black-body to the photometric data, according to $\mathrm{L}_{\text {bol }}=4 \pi \sigma \mathrm{T}^{4} \mathrm{R}^{2}$. We create a two-dimensional grid of temperatures evenly spaced by $20 \mathrm{~K}$, and black body radii $(\mathrm{R})$ in the range of $10^{12}-10^{16} \mathrm{~cm}$ with spacing that corresponds to 0.002 mag. We then compute synthetic photometry from the black body distribution for every $\mathrm{T}$ and $\mathrm{R}$ values in each of the filter bands. Since data in different photometric bands were sometimes taken at different epochs, linear interpolation is used to account for the missing epochs. The interpolation is constrained to a maximum of 10 days from the nearest data point at early or late phases (before day 10 or after day 70), and to 20 days during intermediate phases, where the $\mathrm{SN}$ properties evolve more slowly.

A correct estimation of the photometric uncertainties is needed when fitting a black body to the photometry. Due to the relatively small number of data points, the fit is sensitive to errors that are under- or over-estimated. We therefore set a minimum value of 0.05 magnitudes to the error (such that the error is the maximal value between the given photometric error and $0.05 \mathrm{mag}$ ), which is a typical value for the scatter in our light curves. We assign the effective wavelength of the filter transmission curve to each band, and fit the data to find the black body temperature and radius by minimizing $\chi^{2}$. The uncertainty on the temperature is found by marginalizing the likelihood over the radius and finding the upper and lower temperature where $\chi^{2}=\chi_{\min }^{2}+1$. To find the uncertainty in the luminosity, we calculate $\mathrm{L}_{b o l}$ for every $\mathrm{T}$ and $\mathrm{R}$, and find the contour in which $\chi^{2}=\chi_{\min }^{2}+1$. The maximal and minimal values of $\mathrm{L}_{b o l}$ are taken to be the upper and lower errors, respectively.

The SN spectrum is expected to follow a black body shape only in a limited frequency range, where $\mathrm{h} v \sim \mathrm{kT}$. At high frequencies the flux is suppressed by line blanketing, and at much lower frequencies, in the Rayleigh-Jeans (RJ) regime, it is predicted to be brighter than the RJ tail due

1 The NASA/IPAC Extragalactic Database (NED) is operated by the Jet Propulsion Laboratory, California Institute of Technology, under contract with the National Aeronautics and Space Administration (NASA). 
Table 1. SN Sample Details

\begin{tabular}{|c|c|c|c|c|c|}
\hline SN name & Bands & Explosion day (MJD) & $\mu$ & $\mathrm{z}_{\text {host }}$ & Reference \\
\hline SN1999em & $\mathrm{U}, \mathrm{B}, \mathrm{V}, \mathrm{R}, \mathrm{I}, \mathrm{J}, \mathrm{H}, \mathrm{K}$ & $51476 \pm 4$ & $29.84 \pm 0.05$ & 0.002 & $\begin{array}{l}\text { Leonard et al. (2002a) } \\
\text { Pejcha \& Prieto }(2015)\end{array}$ \\
\hline SN1999gi & $\mathrm{B}, \mathrm{V}, \mathrm{R}, \mathrm{I}$ & $51519 \pm 4$ & $30.24 \pm 0.04$ & 0.002 & Faran et al. (2014a) \\
\hline SN2000dc & $\mathrm{B}, \mathrm{V}, \mathrm{R}, \mathrm{I}$ & $51762 \pm 4$ & $32.93 \pm 0.14$ & 0.010 & Faran et al. (2014a) \\
\hline SN2001cm & $\mathrm{B}, \mathrm{V}, \mathrm{I}$ & $52064 \pm 1$ & $33.18 \pm 0.10$ & 0.011 & Faran et al. (2014a) \\
\hline SN2001cy & $\mathrm{B}, \mathrm{V}, \mathrm{R}, \mathrm{I}$ & $52086 \pm 6$ & $33.01 \pm 0.12$ & 0.015 & Faran et al. (2014b) \\
\hline SN2001do & $\mathrm{B}, \mathrm{V}, \mathrm{R}, \mathrm{I}$ & $52134 \pm 2$ & $32.35 \pm 0.15$ & 0.010 & Faran et al. (2014b) \\
\hline SN2001fa & $\mathrm{B}, \mathrm{V}, \mathrm{R}, \mathrm{I}$ & $52198 \pm 3$ & $34.24 \pm 3.42$ & 0.017 & Faran et al. (2014b) \\
\hline SN2001x & $\mathrm{B}, \mathrm{V}, \mathrm{R}, \mathrm{I}$ & $51963 \pm 5$ & $31.59 \pm 0.11$ & 0.005 & Faran et al. (2014a) \\
\hline SN2002gd & $\mathrm{B}, \mathrm{V}, \mathrm{R}, \mathrm{I}$ & $52553 \pm 15$ & $32.90 \pm 0.21$ & 0.009 & Faran et al. (2014a) \\
\hline SN2003hf & $\mathrm{B}, \mathrm{V}, \mathrm{R}, \mathrm{I}$ & $52864 \pm 2$ & $35.51 \pm 3.55$ & 0.031 & Faran et al. (2014b) \\
\hline SN2003hk & $\mathrm{B}, \mathrm{V}, \mathrm{R}, \mathrm{I}$ & $52860 \pm 2$ & $34.41 \pm 0.20$ & 0.023 & Faran et al. (2014b) \\
\hline SN2003iq & $\mathrm{B}, \mathrm{V}, \mathrm{R}, \mathrm{I}$ & $52920 \pm 2$ & $32.28 \pm 0.08$ & 0.008 & Faran et al. (2014a) \\
\hline SN2003z & $\mathrm{B}, \mathrm{V}, \mathrm{R}, \mathrm{I}$ & $52665 \pm 5$ & $31.23 \pm 3.12$ & 0.004 & Faran et al. (2014a) \\
\hline SN2004A & $\mathrm{B}, \mathrm{V}, \mathrm{R}, \mathrm{I}$ & $53007 \pm 7$ & $31.61 \pm 0.32$ & 0.003 & $\begin{array}{l}\text { Gurugubelli et al. (2008) } \\
\text { Maguire et al. (2010) }\end{array}$ \\
\hline SN2004du & $\mathrm{B}, \mathrm{V}, \mathrm{R}, \mathrm{I}$ & $53228 \pm 2$ & $33.94 \pm 0.13$ & 0.017 & Faran et al. (2014a) \\
\hline SN2004et & $\mathrm{U}, \mathrm{B}, \mathrm{V}, \mathrm{R}, \mathrm{I}, \mathrm{J}, \mathrm{H}, \mathrm{K}$ & $53271 \pm 1$ & $28.41 \pm 0.07$ & 0.000 & Maguire et al. (2010) \\
\hline SN2005cs & Swift UVOT,U,B,V,R,I,J,H,K & $53549 \pm 0$ & $29.36 \pm 0.01$ & 0.002 & Pastorello et al. (2009) \\
\hline SN2006bp & Swift UVOT,U,B,V,r,i & $53834 \pm 1$ & $31.11 \pm 0.05$ & 0.004 & Quimby et al. (2007) \\
\hline SN2007od & Swift UVOT,U,B,V,R,I,J,H,K & $54399 \pm 8$ & $32.29 \pm 0.17$ & 0.006 & Inserra et al. (2011) \\
\hline SN2008in & Swift UVOT,U,B,V,R,I,J,H & $54822 \pm 10$ & $30.52 \pm 0.09$ & 0.005 & Roy \& Kumar (2012) \\
\hline SN2009N & Swift UVOT,B,g,V,R,r,I,i,J,H & $54845 \pm 11$ & $31.68 \pm 0.08$ & 0.003 & Takáts et al. (2014) \\
\hline SN2009bw & Swift UV,U,B,V,R,I,J,H,K & $54917 \pm 3$ & $30.60 \pm 0.02$ & 0.004 & Inserra et al. (2012) \\
\hline SN2009ib & $\mathrm{U}, \mathrm{u}, \mathrm{B}, \mathrm{g}, \mathrm{V}, \mathrm{R}, \mathrm{r}, \mathrm{I}, \mathrm{i}, \mathrm{J}, \mathrm{H}$ & $55041 \pm 10$ & $31.48 \pm 0.31$ & 0.004 & Takáts et al. (2015) \\
\hline SN2012A & Swift UVOT,U,B,g,V,R,r,I,i,J,H,K & $55929 \pm 5$ & $29.72 \pm 0.17$ & 0.003 & Tomasella et al. (2013) \\
\hline SN2012aw & Swift UVOT,U,u,B,g,V,R,r,I,i,J,H,K & $56002 \pm 1$ & $29.89 \pm 0.07$ & 0.003 & $\begin{array}{l}\text { Bose et al. (2013) } \\
\text { Dall'Ora et al. (2014) }\end{array}$ \\
\hline SN2012ec & $\mathrm{u}, \mathrm{B}, \mathrm{g}, \mathrm{V}, \mathrm{R}, \mathrm{i}, \mathrm{J}, \mathrm{H}, \mathrm{K}$ & $56143 \pm 10$ & $31.57 \pm 0.45$ & 0.005 & Barbarino et al. (2015) \\
\hline SN2013ab & Swift UVOT,U,B,g,V,R,r,I,i & $56340 \pm 1$ & $31.71 \pm 0.66$ & 0.005 & Bose et al. (2015a) \\
\hline SN2013by & Swift UV,u,B,g,V,r,i & $56407 \pm 11$ & $30.84 \pm 0.15$ & 0.004 & Valenti et al. (2015) \\
\hline SN2013ej & Swift UV ${ }^{*}, \mathrm{U}, \mathrm{u}, \mathrm{B}, \mathrm{g}, \mathrm{V}, \mathrm{R}, \mathrm{r}, \mathrm{I}, \mathrm{i}$ & $56497 \pm 1$ & $29.77 \pm 2.98$ & 0.002 & $\begin{array}{l}\text { Richmond (2014) } \\
\text { Valenti et al. (2014) }\end{array}$ \\
\hline
\end{tabular}

to the fact that the thermalization depth in this range is frequency dependent (Shussman et al. 2016a). We observe both effects in our data, and fit a black body only to the wavelength regions where it provides a good approximation.

In agreement with the theoretical predictions, we see that at high temperatures JHK observations cannot be well described by a standard black body spectrum, and tend to systematically lie above the RJ tail. This effect was recently modeled analytically by Shussman et al. (2016a) and will be further discussed in section 5.3. In the cases where this discrepancy is observed, we use only UV and optical data, and exclude the JHK bands.

As the temperature drops below 10,000 - 12,000K, line blanketing by iron group elements becomes strong and creates a deficiency in the measured UV flux, compared to a pure black body. The main species responsible for the strong absorption are Fe III and Ti III (Kasen \& Woosley 2009). Line opacity is highly sensitive to the temperature, and even a slight cooling of the photosphere induces a fast recombination of Fe III and Ti III to Fe II and Ti II (Kasen \& Woosley 2009; Eastman et al. 1996). The flux absorption becomes stronger and shifts further to the optical bands as the temperature continues to decrease to $8,000 \mathrm{~K}$. Figure. 1 demonstrates the effect of line blanketing on the SED of SN2012aw on day 41. Data taken at wavelengths shorter than 5000 were found to be affected by line blanketing and were excluded from the fit (grey points), and only bands with wavelengths longer than 5000 were used (red points). The resulting black body at a temperature of $6420 \mathrm{~K}$ fits the red points very well and is also in very good agreement with a spectrum taken at the same epoch. The observed spectrum also confirms the flux cut-off around the $B$-band. In order to determine the time where the flux in each photometric band is suppressed by line-blanketing, we run the black body fitting procedure on each of the following filter groups: UV-UBVRIJHK, UBVRIJHK, BVRIJHK, VRIJHK and RIJHK, i.e., each time excluding the bluest band. We first determine, as an example, when the flux in the UV bands falls below the black body curve by looking at the fit to the UBVRIJHK regime. As long as the temperature is high enough, the UV flux will appear above the black body fit to UBVRIJHK or right on it. However, as the temperature decreases enough such that line blanketing starts to have an effect on the UV flux, the UV data points will drop below the UBVRIJHK curve. We exclude a certain band from the fit when it is $1-\sigma$ below the black body curve. This means that until that epoch we can use the UV-UBVRIJHK bands to determine the black body parameters, and from that day on we can only use the UBVRIJHK bands to fit the data. This procedure is repeated with the other bands to determine when the $\mathrm{U}, \mathrm{B}$, and $\mathrm{V}$ bands are affected by line blanketing and need to be excluded. The transition days co- 
incide with the intersection between the temperature curves calculated with the bluest band, and the one calculated without it. Eventually, we construct the final temperature and luminosity curves using the transitions determined for each of the objects.

At early phases, when the temperature is higher than $10^{4} \mathrm{~K}$, the peak of $\mathrm{F}_{\lambda}$ occurs at wavelengths shorter than 3000 and UV observations are therefore crucial to constrain the fit parameters. JHK observations lie far from the peak of $\mathrm{F}_{\lambda}$ even at low temperatures $(\sim 6000 \mathrm{~K})$, and therefore do not play a critical role in constraining the temperature. However, due to the exclusion of many of the bluer bands by line blanketing, it is necessary to have more data points in the red to improve the fit, meaning that JHK data become important at late epochs.

In order to quantify the importance of UV and InfraRed (IR) photometry, we run a simulation and estimate the expected errors on the temperature in the absence of UV and IR. We produce synthetic photometry from black body distributions at temperatures $5000 \mathrm{~K}-25,000 \mathrm{~K}$ in $1000 \mathrm{~K}$ bins, simulating a spread in the data using the typical photometric errors in each band. We then fit the synthetic data to a black body, repeating the process 100 times per temperature bin. The mean value and standard deviation (STD) of the best-fitting temperatures are computed, where we treat the STD as a measure of the typical statistical error. The uncertainties deduced from the simulation are presented as a function of the temperature in Figure. 2. From the upper panel of Figure. 2 one can see that at temperatures of $\sim 20,000 \mathrm{~K}$, the uncertainties on the temperature are quite high (over $800 \mathrm{~K}$ ) even with UV data. This reflects the fact that $\mathrm{F}_{\lambda}$ peaks at $\sim 1300$, while the effective wavelength of the bluest filter we use (Swift $-u v w 2)$ is only at 2230 . Below $\mathrm{T}=15,000 \mathrm{~K}$, fits that do not include UV (but do include U) are able to reproduce the temperature with an accuracy of $\sim 500 \mathrm{~K}$. When $U$-band data is excluded, the fit reaches that accuracy below $\mathrm{T}=12,000 \mathrm{~K}$.

At lower temperatures, corresponding to late epochs, most of the flux at wavelengths shorter than the B band is already affected by line blanketing and only bands with effective wavelengths longer than the $\mathrm{V}$ band can be used. In the bottom panel of Figure 2 it is evident that JHK data are important at $\mathrm{T}>7000 \mathrm{~K}$ if the $\mathrm{B}$ band is not included, as the STD of the fit temperature is relatively high and rises rapidly with the model temperature. Although fitting with the $\mathrm{V}, \mathrm{R}$ and I bands is still able to produce errors below $10 \%$, we will see in Section 4.1 that the flux in the $\mathrm{V}$ band is typically absorbed by iron blanketing at $\sim 6000 \mathrm{~K}$. In the absence of JHK observations, we are left with only 3 data points for many objects - V, R and I. In these cases, it is impossible to determine when the $\mathrm{V}$ band falls below the black body curve, since we cannot examine the fit done without $\mathrm{V}$, having only 2 data points at longer wavelengths. As a result, when an object does not have JHK data we typically cannot trust the temperature curve below $\sim 6000 \mathrm{~K}$ and we do not fit the data below this temperature.

Throughout this paper, we consider only objects with U or UV data to deduce physical parameters at high temperatures (above $\sim 10,000 \mathrm{~K}$ ), and objects with JHK data at low temperatures (below $\sim 6000 \mathrm{~K}$ ).

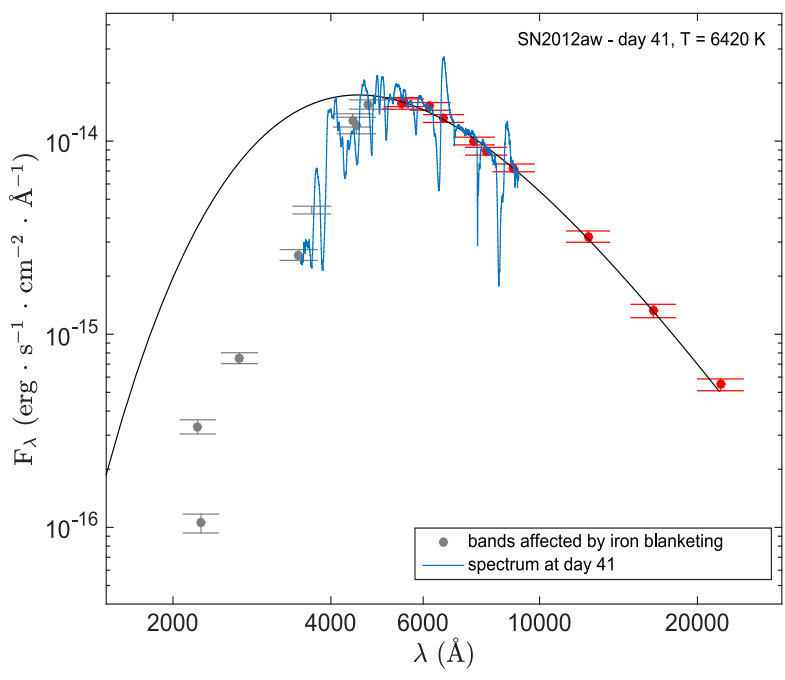

Figure 1. Black body fit of SN2012aw on day 41. The grey points represent bands that are affected by iron-blanketing and were therefore excluded from the fit. The black body curve fits the data very well above 5000, whereas at shorter wavelengths the SED is no longer represented by a black body. A spectrum taken on day 41 (Bose et al. 2013) is also shown to coincide well with the data, and confirms the flux cutoff around the $B$-band.

\section{RESULTS}

\subsection{Temperature}

The temperature curves are computed from the black body fits at each epoch and are presented in Figure. 3 (a list of all the results is also available in Appendix. A). After the explosion, the envelope expands and cools adiabatically. The typical temperatures during the first 10 days are above 10,000 degrees. In cases where UV data exist, the typical errors for that temperature range are smaller than $\sim 500 \mathrm{~K}$, and are comparable to the errors predicted by our simulations (see Section 3). Between 20 and 40 days after the explosion, the temperature curves start evolving more slowly compared to early phases. The flattening typically happens between $6000 \mathrm{~K}$ and $7000 \mathrm{~K}$, and is therefore consistent with being associated with a recombination wave that propagates into the envelope and dictates the black body temperature to be the temperature of hydrogen recombination. This effect is analyzed and discussed in Section 5.2. We note that objects without JHK data do not show this flattening, since as discussed in Section 3, for these SNe we were not able to determine the time where the $V$-band can no longer be used and the fit stops when the temperature reaches $6000 \mathrm{~K}$.

As in Valenti et al. (2016), we observe that excluding UV data from the fit systematically leads to lower temperatures. This is true also for U, B and V, where the temperature produced without the bluest band is lower than that produced with it, before its flux is affected by iron blanketing. Since we do not observe this behavior in the simulation described in Section 3, the effect is not statistical and points to a deviation of the spectrum from a black body. We suggest that this is related to the re-distribution of energy that is absorbed by line blanketing. Most of the absorbed 

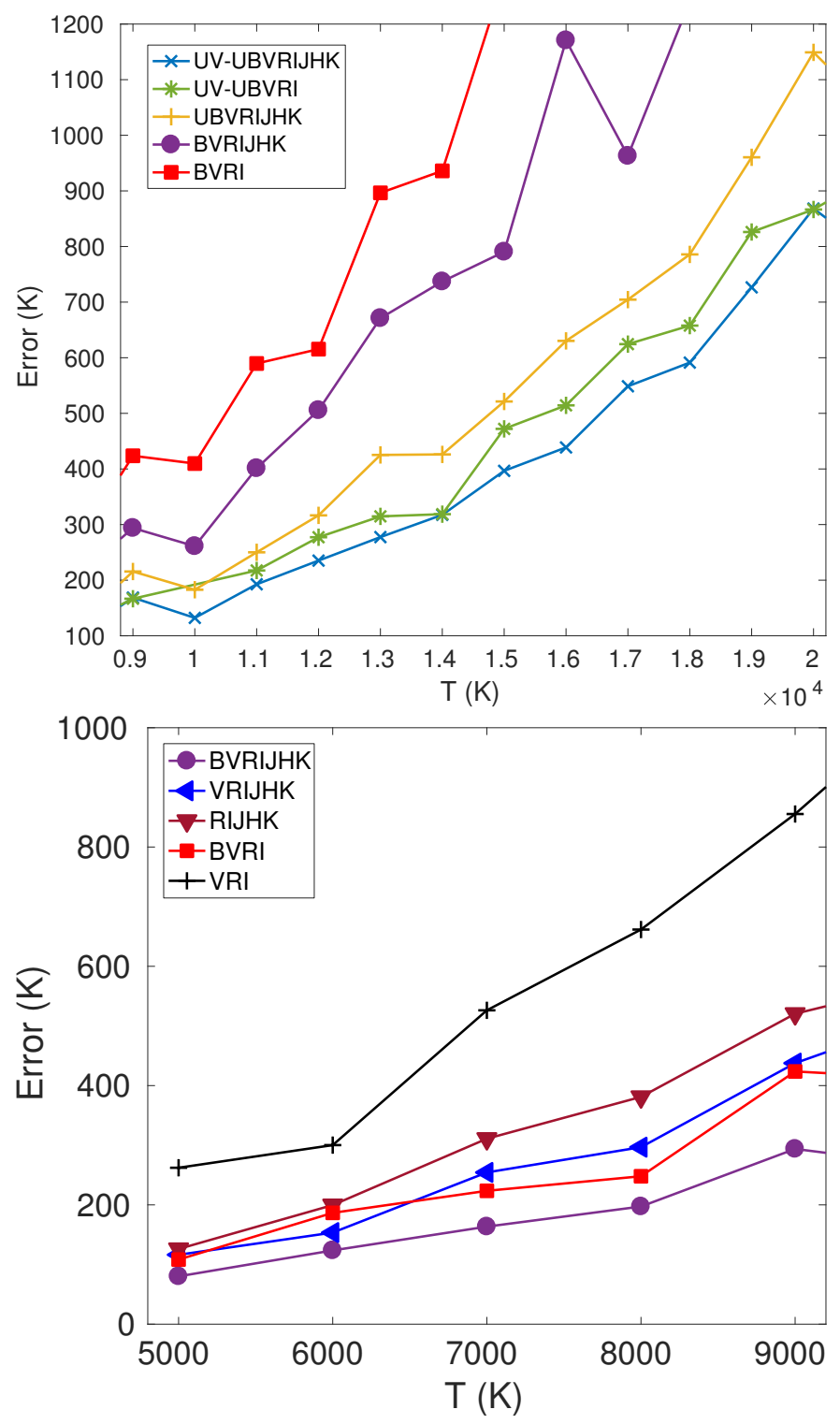

Figure 2. The expected uncertainties of the temperature of a black body, resulting from a simulation of synthetic data. The estimated uncertainties decrease as a function of the proximity of the bands to the black body peak, and therefore rise with the temperature.

radiation is expected to be re-emitted close to the absorption wavelength (see Pinto \& Eastman 2000). As a result, the flux of the bluest band we use will be higher than the black body at the same temperature. Since the bands near the peak of the spectrum have the highest effect on the fit, that will result in higher fit temperatures. We redo the fits without the bluest band, and measure the flux under the resulting black body curve. We then measure the flux excess in the bands that lie above the black body curve, and the flux deficiency (due to line blanketing) in the bands below the black body curve and find that they are of the same order. This reinforces the assumption that the absorbed radiation by iron group elements is emitted at wavelengths close to the black body peak, and may add an uncertainty to the temperature and bolometric luminsoity that we measure. Above $10,000 \mathrm{~K}$, the temperatures calculated with the bluest band are $\approx 10 \%$ higher than the ones calculated when it is omitted, and the difference becomes less significant at lower temperatures. The effect on the luminosity is higher and can get up to $\approx 10-20 \%$. Therefore, the temperatures and bolometric luminosities presented in this paper can be overestimated by up to $\approx 10-20 \%$.

We record the temperatures at which the flux in different bands starts being affected by line blanketing, and find that the typical temperature for UV is $\sim 11,000 \mathrm{~K}$, and $\sim 8000 \mathrm{~K}$ in the $U$ and $B$ bands. The $V$ band seems to be affected around $\sim 6000 \mathrm{~K}$. These results agree with the temperatures shown in Eastman et al. (1996)'s Figure. 7.

Bersten \& Hamuy (2009) fit a black body to the photometry of SN1999em corrected to $\mathrm{A}_{\mathrm{V}}^{\text {host }}=0.18$ and present its temperature and bolometric luminosity curves. After correcting our data to $\mathrm{A}_{\mathrm{V}}^{\text {host }}=0.18$, we extract the temperature curve and compare it to the middle panel in Bersten \& Hamuy (2009)'s Figure. 8. We find a good agreement between the values of the temperature and its evolution. The temperature computed at the first epoch, $\sim 5$ days, is around $13,000 \mathrm{~K}$ in both curves and decreases to show a "bump" around day 16. Eventually, both curves settle on a temperature of $\sim 6000 \mathrm{~K}$ in the middle of the plateau. Valenti et al. (2016) also fit a black body to several SNe that are included in our sample, but unfortunately the values are not provided, and we cannot perform a quantitative comparison.

\subsection{Luminosity}

The bolometric luminosity for each of the SNe is computed from the fit and the curves are presented in Figure. 4. Similarly to the temperature, the luminosity typically decreases as a power law during early epochs. The luminosity in most of the objects relents from its fast decline and starts to decrease more moderately, where the flattening seems to coincide with the break in the temperature. There are 3 objects whose luminosity not only flattens but also starts to rise. This happens for SN2004A and SN2009N at day 30 after explosion, and for SN2005cs at day 23. The transition in luminosity happens quite sharply and occurs when the temperatures are $6000 \mathrm{~K}, 5900 \mathrm{~K}$ and $6900 \mathrm{~K}$ for the 3 objects, respectively. The change in the evolution of the luminosity is probably also related to the recombination of the envelope. We will discuss this further in Section 5. At the end of the plateau, the bolometric luminosity falls sharply.

We compare our bolometric luminosity curves to pseudo-bolometric curves from the literature by correcting for the different assumed distances to the SNe, and shifting in time to match the assumed explosion day. While broadly speaking there is mostly agreement between our work and previous efforts, there are still some discrepancies. The comparison is presented in Figure. 5. Pseudo-bolometric luminosities that were not computed with UV nor JHK data, as done for SN2009bw, SN2008in, and SN2004A, can be underestimated by up to $30 \%$.

\subsection{The Effect of Extinction}

Host interstellar or circumstellar dust can introduce extinction that is not corrected for in our data (see Section 2), 
Faran et al.

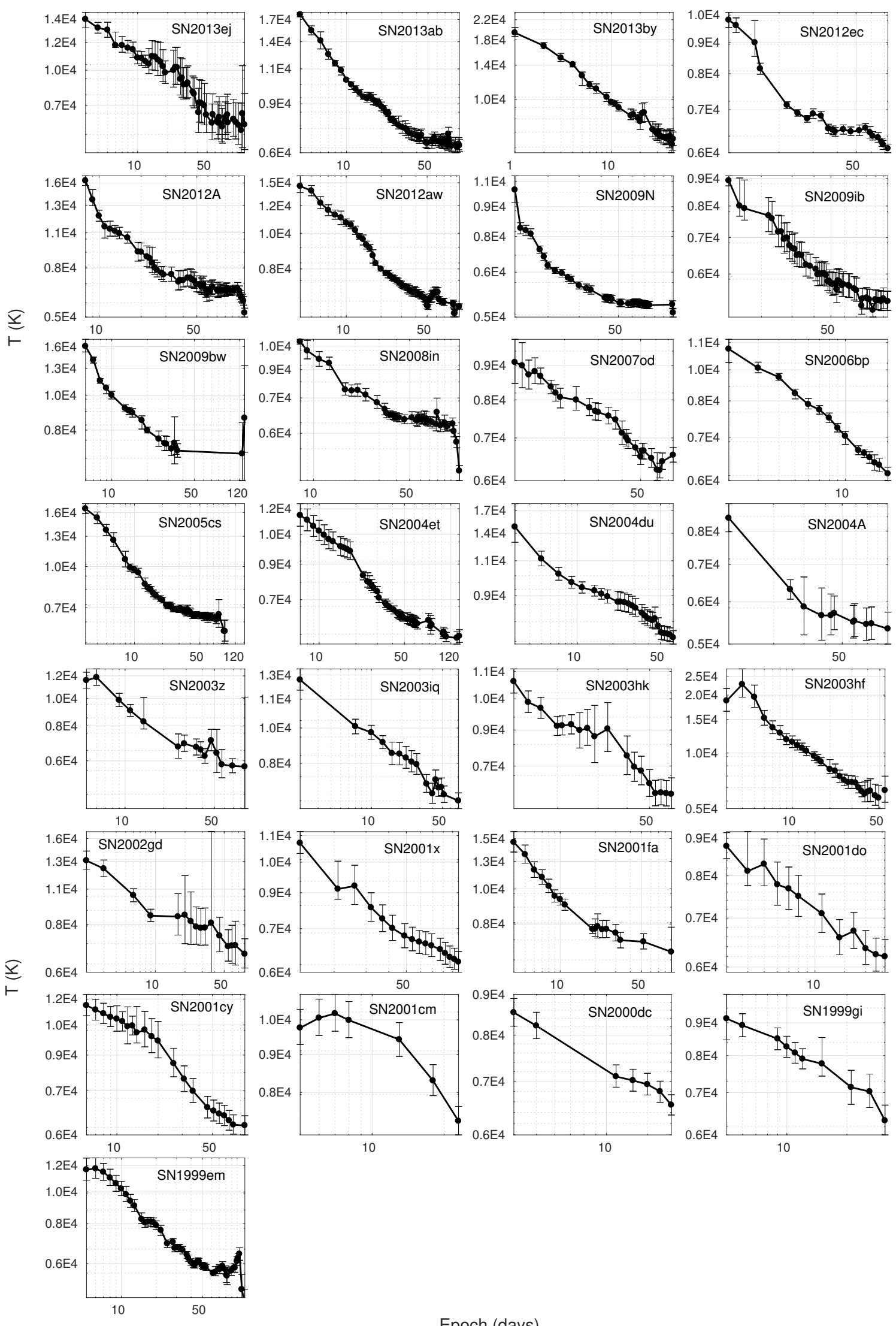

Epoch (days)

Figure 3. The temperature as a function of time for each SN in the sample. 


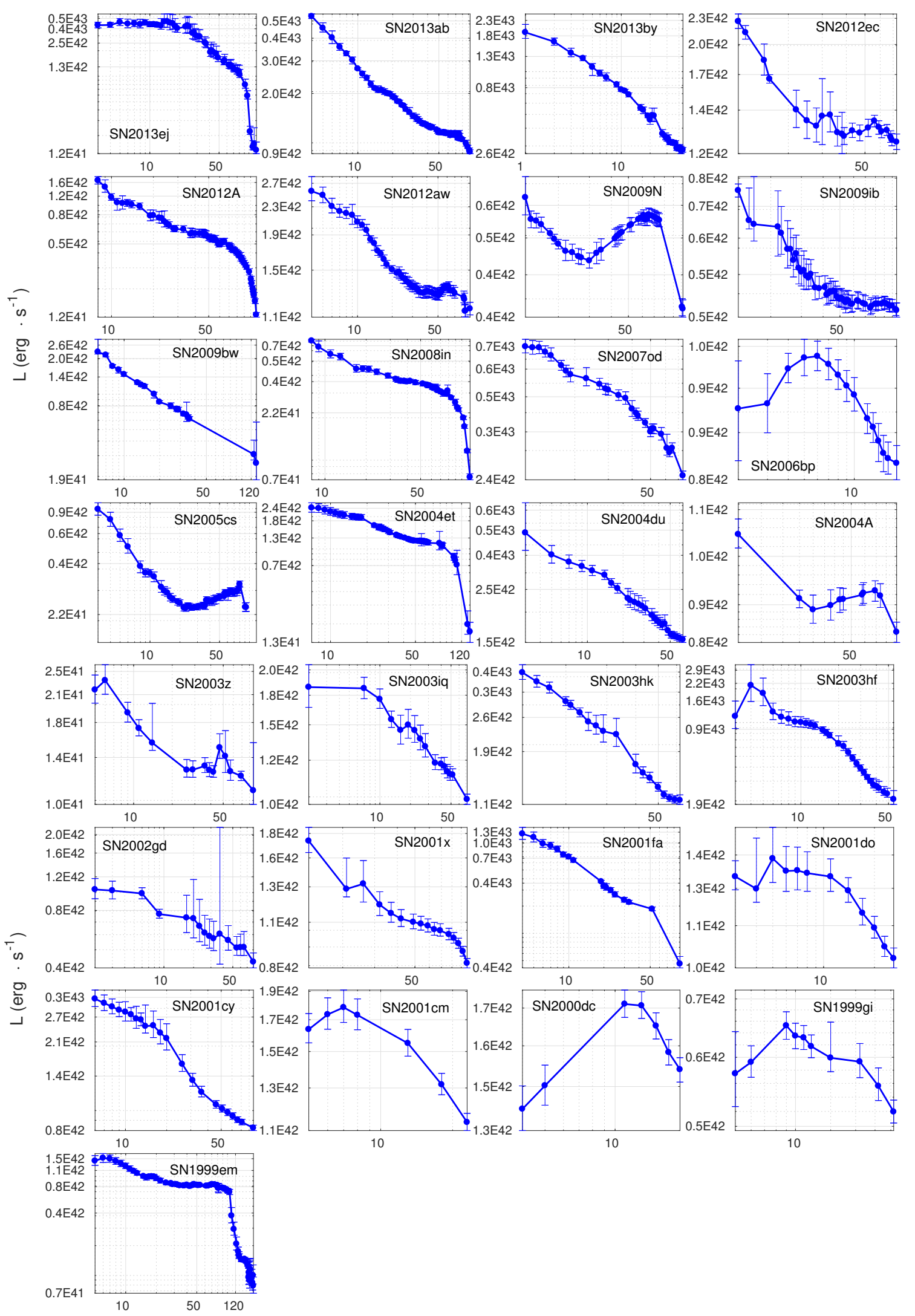

Epoch (days)

Figure 4. The bolometric luminosity curves as calculated from the black body fits for each SN in the sample. 


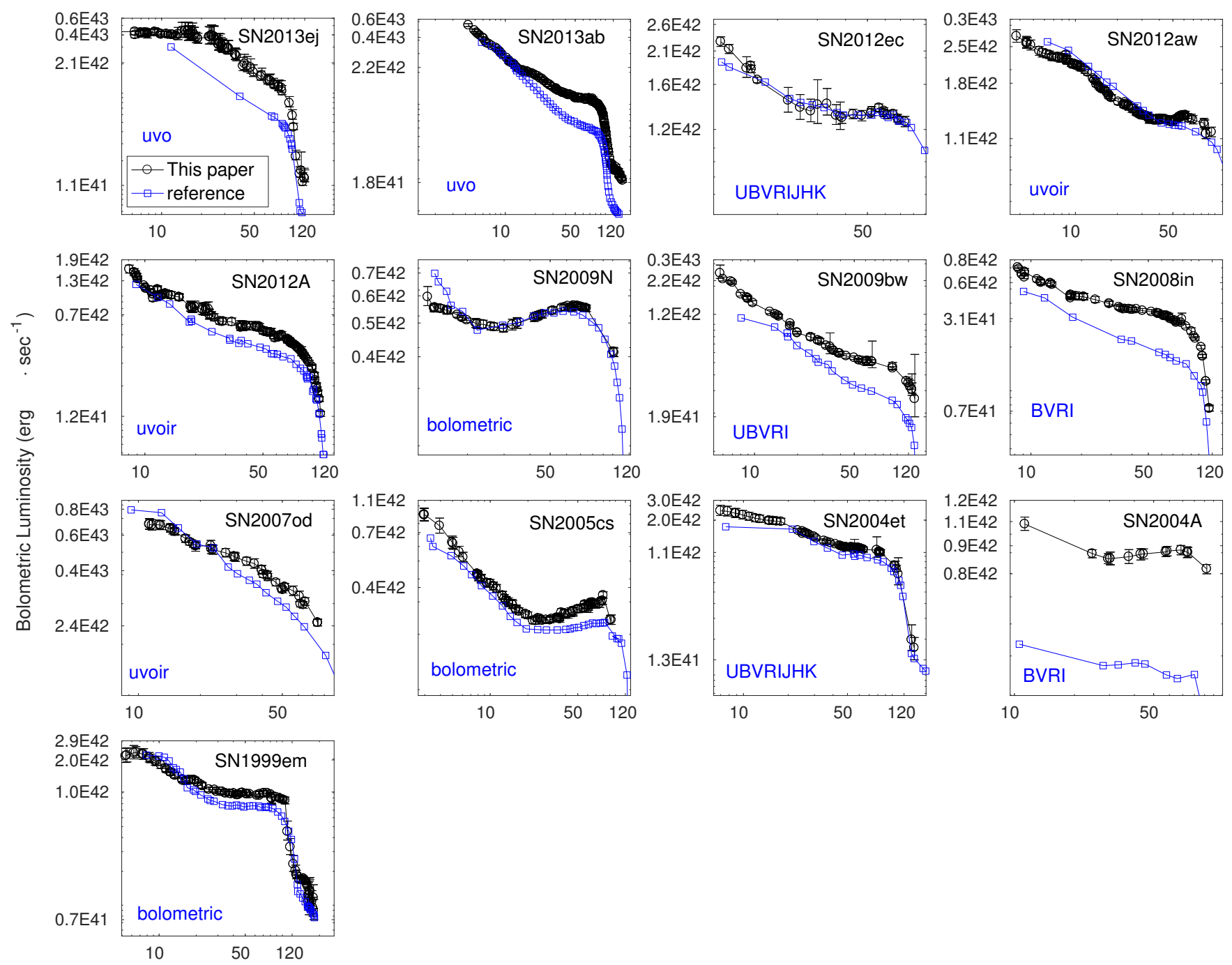

Phase (day)

Figure 5. Comparison between the bolometric luminosity curves calculated in this paper (blue) and pseudo-bolometric curves from the literature (red), where the integrated wavelength range is specified. 'bolometric' light curves include bolometric corrections or are based on black body fits. Otherwise, the light curves are the integrated luminosity in the observed bands without any bolometric corrections. The luminosity of SN1999em presented here was calculated after correcting for $\mathrm{A}_{\mathrm{V}}^{\text {host }}=0.18$, in order to compare to Bersten \& Hamuy (2009). Discrepancies between the curves at early times are probably due to missing UV flux in the pseudo-bolometic curves. SN2012ec (Barbarino et al. 2015), SN2012aw (Dall'Ora et al. 2014), SN2012A (Tomasella et al. 2013), SN2013ab (Bose et al. 2015a), SN2009N (flux in RJ was approximated by RJ tail, no corrections in the blue) (Takáts et al. 2014), SN2009bw (Inserra et al. 2012), SN2008in (Roy \& Kumar 2012), SN2005cs (bolometric corrections) (Pastorello et al. 2009), SN2004et (Maguire et al. 2010), SN2004A (Hendry et al. 2006; Maguire et al. 2010), SN1999em (Bersten \& Hamuy 2009), SN2007od (Inserra et al. 2011), SN2013ej (Bose et al. 2015b)

resulting in an underestimation of the fit temperatures and luminosities. Although it is quite difficult to find a good estimation for $A_{V}^{\text {host }}$, it is possible to quantify the effect a certain Av value has on the fit parameters as a function of the temperature. We repeat the fitting procedure two more times assuming $\mathrm{E}(\mathrm{B}-\mathrm{V})=0.1$ and 0.05 , and $\mathrm{R}_{V}=3.1$, using the galactic extinction laws of Cardelli et al. (1989). As most type-II $\mathrm{SNe}$ in or sample are expected to have $\mathrm{E}(\mathrm{B}-\mathrm{V})^{\text {host }}<0.1$ (Faran et al. 2014a), this value is effectively an upper limit on the possible required corrections.

In Figure 6 we present the relation between the best fit temperatures resulting from the correction to $\mathrm{E}(\mathrm{B}-\mathrm{V})^{\text {host }}=$ $0.1\left(\mathrm{~A}_{\mathrm{V}}^{\text {host }} \approx 0.3\right)$ and $\mathrm{E}(\mathrm{B}-\mathrm{V})^{\text {host }}=0.05\left(\mathrm{~A}_{\mathrm{V}}^{\text {host }} \approx 0.1\right)$ as a function of the uncorrected SN temperatures. The dependence of the corrected temperatures on $\mathrm{T}\left(\mathrm{A}_{\mathrm{V}}^{\text {host }}=0\right)$ can be well described by a third order polynomial, according to the following relations:

$$
T\left(A_{V}=0.3\right) \approx 3.1 T_{3}^{3}-58 T_{3}^{2}+1630 T_{3}-1730
$$

and:

$$
T\left(A_{V}=0.15\right) \approx 0.69 T_{3}^{3}-9.14 \times 10^{-2} T_{3}^{2}+1150 T_{3}-424,
$$

where $\mathrm{T}_{3} \equiv \mathrm{T}(\mathrm{Av}=0) / 10^{3}$. These relations offer a convenient way to estimate the error on a fit temperature, in the typical extinction range of $\mathrm{A}_{\mathrm{V}}^{\text {host }}=0-0.3 \mathrm{mag}$. 


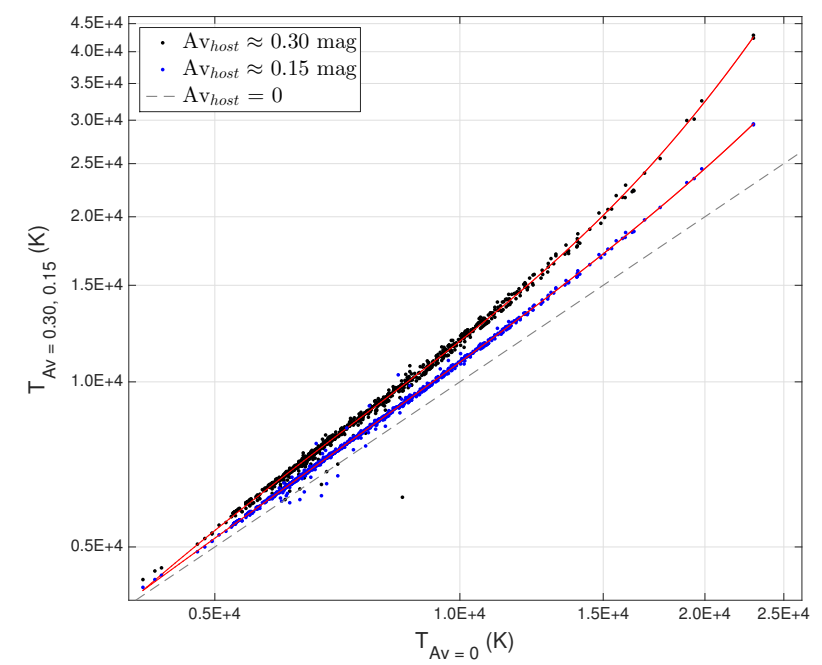

Figure 6. The best fit temperatures resuting from an extinction correction of $\mathrm{A}_{\mathrm{V}}^{\text {host }}=0.3$ (black dots) and $\mathrm{A}_{\mathrm{V}}^{\text {host }}=0.15$ (blue dots), as a function of $\mathrm{T}\left(\mathrm{A}_{\mathrm{V}}^{\text {host }}=0\right)$. The grey dashed line indicates $\mathrm{T}=$ $\mathrm{T}_{\mathrm{A}_{\mathrm{V}}^{\text {host }}=0}$. The third order polynomial fits to the data are plotted in red and can be used to translate between the uncorrected and corrected temperatures.

Since the effect of extinction on the RJ is weak, we expect the luminosity to behave as $\frac{L_{A_{V}}}{L_{0}}=\left(\frac{T_{A_{V}}}{T_{0}}\right)^{3}$ at high temperatures. We fit the data with $\mathrm{T}\left(\mathrm{A}_{\mathrm{V}}=0\right)>8000 \mathrm{~K}$ according to this relation for both $A v=0.3$ and $A v=0.15$ and present the data and the fit in Figure. 7. This, together with the previous relation for the temperatures, allows also the bolometric luminosity to be corrected for extinction as the relation holds down to low temperatures of $\sim 8000 \mathrm{~K}$. Below that temperature, the corrections to $\mathrm{L}$ are less than $10 \%$, which is of the order of the uncertainty.

\section{COMPARISON TO THEORY}

\subsection{The Temperature at the Beginning of the Plateau}

The formation process of the plateau in Type-II SNe and the origin of its shape (i.e., its luminosity and temperature evolution) are not fully understood. The common wisdom states that the plateau is formed due to a recombination wave that propagates into the envelope in Lagrangian coordinates. The recombination front defines the photosphere and therefore also fixes its temperature to the temperature of hydrogen recombination in the envelope. According to this view, the plateau should start when $T \approx 7500 \mathrm{~K}$. However, more detailed theoretical models show that the peak in each photometric band is observed slightly before the black body peak enters the observed band. This is why redder bands peak at later time. Recombination prevents the observed temperature from falling below $\sim 6000 \mathrm{~K}$, which is the main reason that after the peak the luminosity in the optical and IR bands falls rather slowly, and creates what is referred to as the plateau. We therefore expect to find photospheric temperatures higher than $7500 \mathrm{~K}$ when the plateau starts.

We define the plateau starting time, $t_{p}$, in a specific band to be the day at which the light curve changes by less

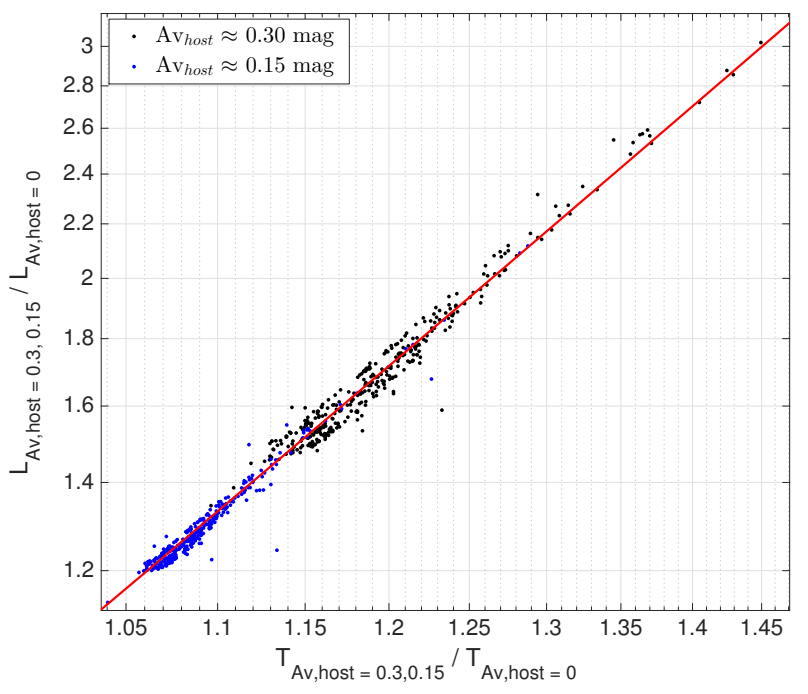

Figure 7. The ratio between the extinction corrected $\mathrm{L}_{b o l}$ $\left(\mathrm{A}_{\mathrm{V}}^{\text {host }}=0.3\right.$ in black and $\mathrm{A}_{\mathrm{V}}^{\text {host }}=0.15$ in blue $)$ and the uncorrected $\mathrm{L}_{b o l}$, as a function of the similar temperature ratio for $\mathrm{T}_{\mathrm{A}_{\mathrm{V}}^{\text {host }}=0}>8000 \mathrm{~K}$. Since extinction has a minimal effect on long wavelength observations, the Rayleigh-Jeans part of the spectrum at high temperatures is expected to be approximately fixed. Therefore, the data follow the relation: $\frac{L_{A_{V}}}{L_{0}}=\left(\frac{T_{A_{V}}}{T_{0}}\right)^{3}$, which is presented by the red line.

than 0.02 magnitudes per day. To find $t_{p}$, we fit a low order polynomial to the first 15-20 days and find the day where the derivative equals $0.02 \mathrm{mag} /$ day. In order to estimate the uncertainties in $t_{p}$, we use the photometric errors of the data to generate random Gaussians errors, from which we create simulated data. We run the fit 1000 times on simulated data and use the mean of the results as the value of $t_{p}$ and the standard deviation as its uncertainty. The value of $t_{p}$ can be sensitive to the order of the polynomial and to the time range chosen for the fit. The maximal discrepancies introduced by changing those parameters are typically not larger than one day. We therefore set a minimal error of one day on $t_{p}$.

In Table 2 we present the $t_{\mathrm{p}}$ values computed in the $R$ and $I$ bands. Some objects have only an upper limit on $t_{\mathrm{p}}$, since they were first observed already on the plateau. Nevertheless, for most of the objects it is clear that the plateau in $R$ starts slightly before the plateau in $I$, as predicted by theory. In Figure 8 we demonstrate the different locations of the plateau in the $R$ and in the $I$ band for SN2012aw.

The temperatures associated with $\mathrm{t}_{\mathrm{p}}$ in $R$ and $I$ are computed by interpolating the temperature curves to $t_{\mathrm{p}}$. We plot the temperatures at $\mathrm{t}_{\mathrm{p}}$, i.e. $\mathrm{T}_{\mathrm{p}}=T\left(t=t_{p}\right)$ in the $R$-band for each $\mathrm{SN}$ in Figure. 9. The blue arrows indicate the effect that $\mathrm{A}_{\mathrm{V}}^{\text {host }}=0.3$ would have on $\mathrm{T}_{p}$ at $\mathrm{T} \approx 8000 \mathrm{~K}$ and $\mathrm{T} \approx 11,000 \mathrm{~K}$, according to equation 1 . Objects with only lower limits (i.e., first data point lies already on the plateau) are presented by red triangles. Almost all $\mathrm{T}_{p}$ values lie above $8000 \mathrm{~K}$, and many of them above $10,000 \mathrm{~K}$. The low luminosity SN2005cs shows an exceptionally high lower limit of $T_{\mathrm{p}} \gtrsim 16,500 \mathrm{~K}$. The observed range of $T_{p}$ (with the exception of SN2005cs) is consistent with the theoretical light curves prediction by Shussman et al. (2016a). For example, the predicted $R$-band $T_{p}$ for explosion energy of $10^{51} \mathrm{erg}$ of progenitors with radii 


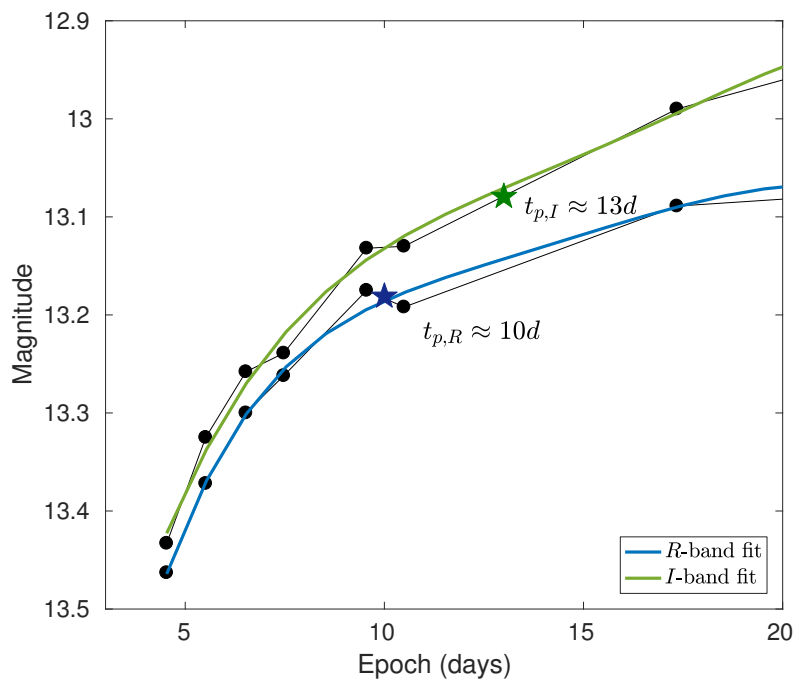

Figure 8. The locations of $\mathrm{t}_{p, R}$ and $\mathrm{t}_{\boldsymbol{p}, \boldsymbol{I}}$ of SN2012aw, as defined by the derivative of the polynomial fit to the $R$-band (blue) and the $I$-band (green). The plateau in the $I$-band appears to start slightly later than the plateau in the $R$-band.

in the range of $400-800 R_{\odot}$ and ejecta masses in the range of $7-15 M_{\odot}$ is between about $10,000 \mathrm{~K}$ and $12,000 \mathrm{~K}$.

\subsection{Signs of Recombination in the Temperature and Luminosity Curves}

As discussed in Sections 4.1 and 4.2, the evolution of the temperature and the bolometric luminosity is characterized well by a power-law, that flattens when the temperature drops to $\sim 6000-7000 \mathrm{~K}$. We compute the early values of the logarithmic derivatives of the luminosity and temperature, $\alpha_{\mathrm{L}}$ and $\alpha_{\mathrm{T}}$, respectively, during the first 15 days after the explosion. SNe that do not have U or UV data are excluded, since the temperatures at these epochs are typically higher than $12,000 \mathrm{~K}$, where U-band data (or bluer) are important to constrain the fit (see Section 3). We also calculate the late logarithmic derivatives between 40 and 100 days, while the SN light curve is on the plateau. For this we choose only SNe with IR data for the reasons discussed in Section 3. The results are summarized in Table 3. The best fit values for the early power law are highly sensitive to the exact value of the zero point in time. Since we make conservative explosion day estimates (see Section. 2), some of the uncertainties on the explosion day are as large as 5-10 days, and introduce non-negligible uncertainties to the values of the power law. The uncertainty values introduced from the fit itself and from the uncertainty on the explosion day are presented separately in Table 3 . The values in the parentheses are the errors produced by the fit, and the upper and lower values are the differences from the $\alpha$ values that we get using the lower and upper boundaries of the explosion day estimate, respectively. In cases where the explosion day uncertainty is large (as in SN2013by, SN2012ec, SN2009jb and SN2008in) the upper and lower uncertainties are quite large. However, the explosion day uncertainty naturally has very little effect on the late values of $\alpha$.

An example of the fit for $\alpha_{T}$ is shown in Figure. 10. It depicts the temperature curve of SN2005cs, on a loga- rithmic sacale. The best-fit logarithmic derivative computed during the first 15 days is $\alpha_{\mathrm{T}}=-0.47 \pm 0.03$, and during days 40-100 is $\alpha_{\mathrm{T}}=-0.06 \pm 0.07$. There is a clear flattening of the temperature curve between $\mathrm{t}=19 \mathrm{~d}$ and $\mathrm{t}=35 \mathrm{~d}$, when the temperature is between $6500 \mathrm{~K}$ and $7500 \mathrm{~K}$. In Figure 11 we present the temperature curves and the power law fits for all the objects that have both UV and IR data. From the values of the logarithmic derivatives (table 3 ) it is clear that at some point the temperature evolution flattens. At early time most values are in the range $\alpha_{\mathrm{T} \text {,early }} \sim-0.6--0.2$ while at late time all best fit values are in the range $\alpha_{\mathrm{T}, \text { late }} \sim-0.15-$ 0 . The weighted mean values of the logarithmic derivatives are $\bar{\alpha}_{\text {T,early }}=-0.38 \pm 0.01$ and $\bar{\alpha}_{\text {T,late }}=-0.08 \pm 0.02$. Although it is not possible to point out the exact temperature of the transition, one can see that the range of temperatures between the two power law regimes is $\sim 6000-7000 \mathrm{~K}$, which is the temperature range expected from hydrogen recombination in type-II SN envelopes.

We also calculate the early and late logarithmic derivatives of $\mathrm{L}_{\mathrm{bol}}$. Similar to the temperature, the bolometric luminosity curves generally have a higher logarithmic derivative in the early phases. Most of the values of $\alpha_{\mathrm{L} \text {,early }}$ are between -0.2 and -0.8 , while most values of $\alpha_{\mathrm{L}, \text { late }}$ are between -0.6 and 0.2 , with weighted mean values of $\bar{\alpha}_{\mathrm{L} \text {,early }}=$ $-0.46 \pm 0.01$ and $\bar{\alpha}_{\mathrm{L}, \text { late }}=-0.22 \pm 0.03$. When including the effect of extinction, we find that the values of $\alpha_{\mathrm{T}}$, early and $\alpha_{\mathrm{L}}$, early increase by $\sim 0.1$ and $\sim 0.2$ respectively, with an extinction value of $\mathrm{E}(\mathrm{B}-\mathrm{V})=0.1 \mathrm{mag}$. This result is also consistent with the expectation from recombination which is expected to cause a flatter, or even rising, light curves once the recombination front reaches facilitate the release of radiation from inner regions.

An interesting question is whether there are correlations between the early and late evolution, or between temperature and luminosity evolution. In Figure 12 we plot $\alpha_{\mathrm{T}, \text { late }}$ vs. $\alpha_{\text {T,early }}$ (upper panel) and $\alpha_{\mathrm{L} \text {,late }}$ vs. $\alpha_{\mathrm{L} \text {,early }}$ (lower panel). The color coding refers to the decline rate of the $V$-band light curve per 100 days, calculated by linearly fitting the magnitude decline rate between day 25 and 75 . The figures show no clear correlations between early and late evolution or between the late decline rate and the temperature evolution (early or late). However, there is a linear correlation between $\alpha_{\mathrm{T}}$ and $\alpha_{\mathrm{L}}$.

Shussman et al. (2016a) provides theoretical predictions of $\alpha_{\mathrm{T} \text {,early }}$ and $\alpha_{\mathrm{L} \text {,early }}$ based on numerical simulations of SN explosions of a large set of RSG progenitors. They find that before recombination $\alpha_{\mathrm{T}}$ is not strictly constant, and that it makes a transition from about -0.35 to -0.6 . The time of steepening in $\alpha_{\mathrm{T}}$ depends on the progenitor radius and ejecta velocity and for typical parameters it ranges between a day and two weeks. Since the data we have is not detailed enough to see the transition between the two power-laws, but only a single average power-law index, the analytic prediction is $\alpha_{\text {T,early }} \approx-0.35--0.6$. This range is marked in figure 12 and it is broadly consistent with the observed values listed in table 2 . The theoretical model for the luminosity evolution predicts $\alpha_{\mathrm{L} \text {,early }} \approx-0.35$. This value depends slightly on the progenitor radius (up to about \pm 0.05 ) and more strongly on the progenitor structure (i.e., density profile). comparison of this prediction to the values listed in table 2 shows that they are consistent for most SNe but not for all. Moreover, the value of $\alpha_{\mathrm{L} \text {,early }}$ is inconsistent with being similar to all 


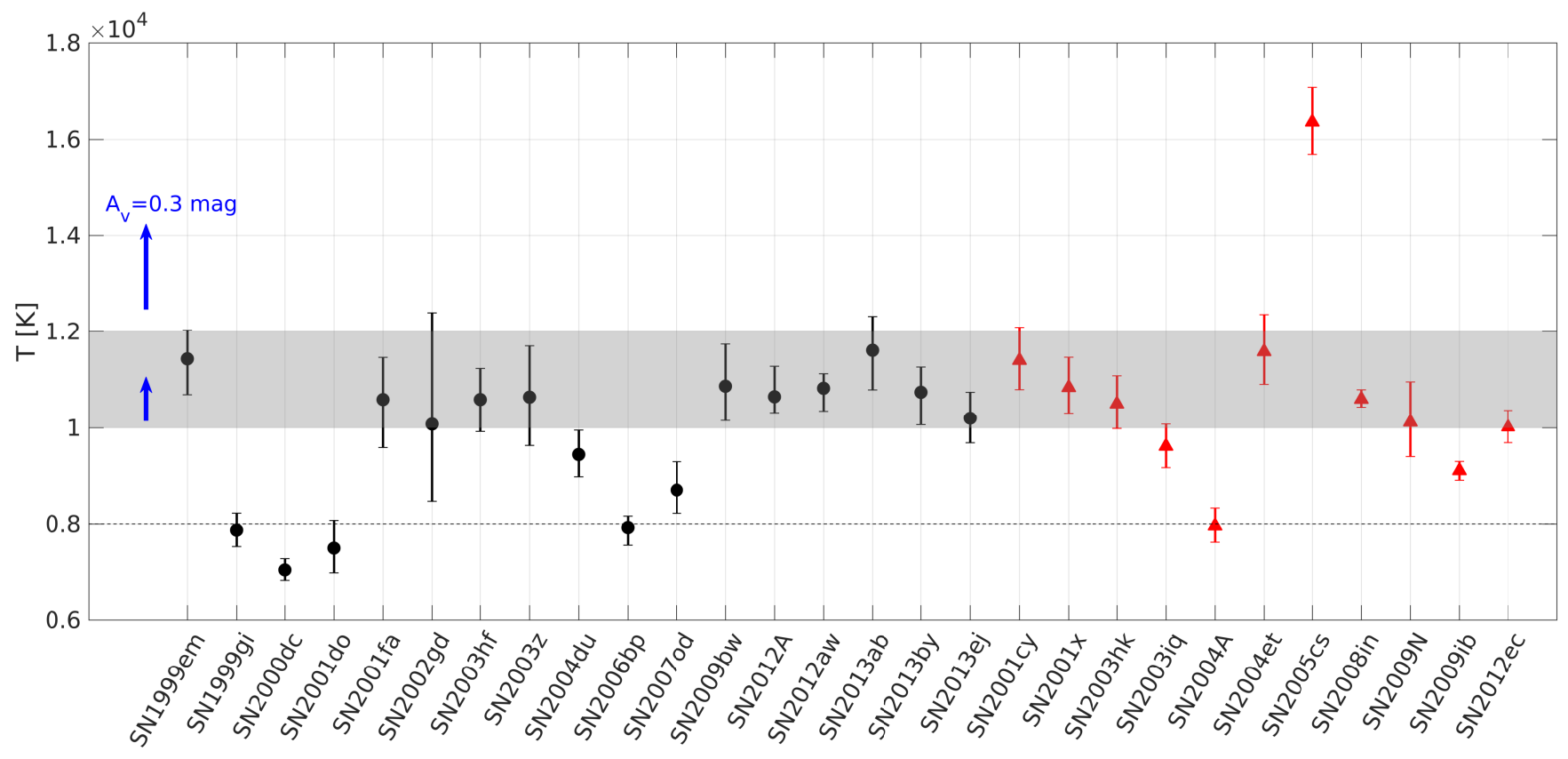

Figure 9. The temperatures at the onset of the plateau phase in the $R$ band. Lower limits are marked by red triangles. The onset of the plateau is defined as the day in which the light curve changes by less than 0.02 magnitudes per day. Most of the temperatures lie above $8000 \mathrm{~K}$, which reinforced our claim that the flattening of the light curve is not caused by recombination. The blue arrows show the effect of extinction on $\mathrm{T}_{p}$ at $\mathrm{T} \approx 11000 \mathrm{~K}$ and $\mathrm{T} \approx 8000 \mathrm{~K}$ for $\mathrm{A}_{V}=0.3$. The plateau temperatures agree with radiative transfer results calculated by Shussman et al. (2016b) for a set of 124 RSGs, represented by the shaded area.

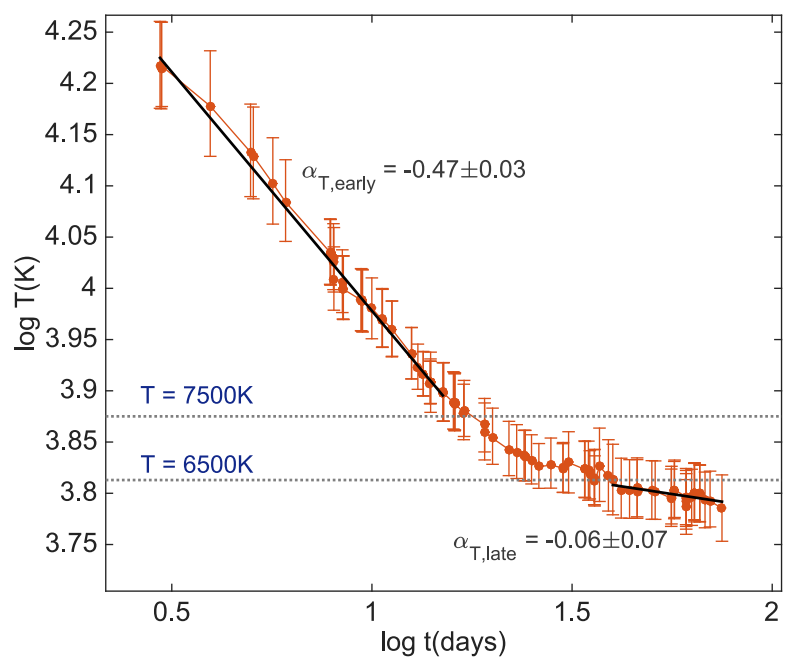

Figure 10. The temperature curve of SN2005cs on logarithmic scale. The temperature behaves as a broken power law, where in early phases it declines rapidly with a power of $-0.47 \pm 0.03$, and at late phases the power law is $-0.06 \pm 0.07$. This transition in the power law happens within the temperature range $6500-7500 \mathrm{~K}$, and we interpret it as the time when the color shell reaches the recombination temperature.

SNe. This is probably due to different density profiles of the progenitors.

\subsection{Deviation from Black Body}

At high temperatures, we find that a black body is not able to describe the whole observed spectrum not only at short wavelengths, where line blanketing is important, but also at long wavelengths on the RJ tail. In the left panel of Figure. 13 we show the SED of SN2012A on day 8, where the temperature is $\sim 15,000 \mathrm{~K}$. The dashed blue line is the best fit to all of the data points, which clearly fails to fit the JHK observations. This effect is observed in all SNe that have early JHK data, which always seem to be brighter than the RJ tail at the temperature corresponding to the optical and UV flux.

Deviation of the RJ tail from a black body spectrum of early type II emission was seen in numerical simulations (e.g., Tominaga et al. 2011) and was modeled recently analytically by Shussman et al. (2016a). According to the model the reason for the deviation is that the flux at different wavelengths is determined at different locations in the outflow at different gas temperatures. Shussman et al. (2016a) find that on the RJ tail the modified spectrum can be approximated as $F_{\boldsymbol{v}} \propto v^{1.4}$ and they also provide an approximation for the entire spectrum. The black solid line in the left panel of Figure. 13 is the best fit of Shussman et al. (2016a) model to the data of SN2012A. The model seems to fit the data very well throughout the UV and the IR and follows the JHK flux where it departs from a standard black body.

At lower temperatures both the standard black body model and the modified black body models are able to describe the JHK observations, as seen in the right panel of Figure. 13. The reason is that these bands are closer to the peak of the spectrum, where the models are essentially 


\begin{tabular}{|c|c|c|c|c|}
\hline SN name & $\mathrm{t}_{p} R$-Band & $\mathrm{T}_{p} R$-band & $\mathrm{t}_{p} I$-Band & $\mathrm{T}_{p} I$-band \\
\hline SN1999em & $8.0(1.0)$ & $11400_{-800}^{+600}$ & $8.0(1.1)$ & $11400_{-800}^{+700}$ \\
\hline SN1999gi & $10.0(1.0)$ & $7900_{-400}^{+400}$ & $10.0(1.0)$ & $7900_{-400}^{+400}$ \\
\hline SN2000dc & $13.0(1.0)$ & $7000_{-300}^{+300}$ & $13.0(1.0)$ & $7000_{-300}^{+300}$ \\
\hline $\mathrm{SN} 2001 \mathrm{~cm}$ & & & $6.0^{*}$ & $9600_{-500}^{+500 *}$ \\
\hline SN2001cy & $6.0^{*}$ & $11400_{-700}^{+700 *}$ & $6.0^{*}$ & $11400_{-700}^{+700 *}$ \\
\hline SN2001do & $8.0(1.0)$ & $7500_{-600}^{+600}$ & $11.0(1.0)$ & $7100_{-500}^{+600}$ \\
\hline SN2001fa & $8.0(1.0)$ & $10600_{-1000}^{+900}$ & $9.0(1.0)$ & $10000_{-700}^{+1000}$ \\
\hline SN2001x & $13.0^{*}$ & $10800_{-600}^{+700 *}$ & $13.0^{*}$ & $10800_{-600}^{+700 *}$ \\
\hline SN2002gd & $6.0(3.0)$ & $10000_{-1700}^{+2400}$ & $6.0(3.0)$ & $10000_{-1700}^{+2400}$ \\
\hline SN2003hf & $13.0(1.0)$ & $10600_{-700}^{+700}$ & $14.0(1.1)$ & $10400_{-700}^{+700}$ \\
\hline SN2003hk & $15.0^{*}$ & $10500_{-600}^{+600 *}$ & $16.0^{*}$ & $10500_{-600}^{+600 *}$ \\
\hline SN2003iq & $7.0^{*}$ & $9600_{-500}^{+500 *}$ & $7.0^{*}$ & $9600_{-500}^{+500 *}$ \\
\hline SN2003z & $9.0(1.5)$ & $11000_{-1100}^{+1100}$ & $12.0(1.6)$ & $9300_{-800}^{+700}$ \\
\hline SN2004A & $11.0^{*}$ & $8000_{-400}^{+400 *}$ & $11.0^{*}$ & $8000_{-400}^{+400 *}$ \\
\hline SN2004du & $10.0(1.0)$ & $9500_{-500}^{+500}$ & $11.0(1.0)$ & $9300_{-500}^{+500}$ \\
\hline SN2004et & $9.0^{*}$ & $11600_{-700}^{+800 *}$ & $9.0^{*}$ & $11600_{-700}^{+800 *}$ \\
\hline SN2005cs & $3.0^{*}$ & $16400_{-700}^{+800 *}$ & $3.0^{*}$ & $16400_{-700}^{+800 *}$ \\
\hline SN2006bp & $8.0(1.0)$ & $7900_{-400}^{+300}$ & $8.0(1.0)$ & $7900_{-400}^{+300}$ \\
\hline SN2007od & $9.0(1.0)$ & $8700_{-600}^{+600}$ & $9.0(1.0)$ & $8700_{-600}^{+600}$ \\
\hline SN2008in & $9.0^{*}$ & $10600_{-200}^{+200 *}$ & $9.0^{*}$ & $10600_{-200}^{+200 *}$ \\
\hline SN2009N & $14.0^{*}$ & $10100_{-800}^{+900 *}$ & $14.0^{*}$ & $10100_{-800}^{+900 *}$ \\
\hline SN2009bw & $9.0(1.0)$ & $10900_{-800}^{+900}$ & $11.0(1.0)$ & $9800_{-600}^{+600}$ \\
\hline SN2009ib & $13.0^{*}$ & $9100_{-300}^{+200 *}$ & $13.0^{*}$ & $9100_{-300}^{+200 *}$ \\
\hline SN2012A & $13.0(1.7)$ & $10600_{-400}^{+700}$ & $14.0(1.0)$ & $10400_{-500}^{+600}$ \\
\hline SN2012aw & $10.0(1.1)$ & $10800_{-500}^{+300}$ & $13.0(3.1)$ & $10000_{-1200}^{+900}$ \\
\hline SN2012ec & $15.0^{*}$ & $10000_{-400}^{+400 *}$ & $15.0 *$ & $10000_{-400}^{+400 *}$ \\
\hline SN2013ab & $9.0(1.0)$ & $11600_{-900}^{+800}$ & $9.0(1.0)$ & $11600_{-900}^{+800}$ \\
\hline SN2013by & $8.0(1.0)$ & $10700_{-700}^{+600}$ & $8.0(1.0)$ & $10700_{-700}^{+600}$ \\
\hline SN2013ej & $12.0(1.6)$ & $10200_{-600}^{+600}$ & $11.0(1.4)$ & $10400_{-600}^{+600}$ \\
\hline
\end{tabular}

Table 2. The times at which the $R$ - and the $I$-bands enter the plateau phase, and the corresponding temperatures at those epochs.

equivalent. Also, at lower temperatures, where recombination starts affecting the spectrum the analytic model is no longer applicable.

\section{SUMMARY}

We calculated the temperaures and bolometric luminosities of 29 type-II SNe, by fitting black body models to their SEDs. We use the results to study the properties at the beginning of the plateau, to look for the signature of hydrogen recombination and to compare the observation before recombination becomes important to theoretical models. Our main findings are listed below.

- The temperature at the onset of the plateau phase in the $R$-band is above $8000 \mathrm{~K}$ for all $\mathrm{SNe}$ in our sample, and exceeds $10,000 \mathrm{~K}$ in many of them. This temperature changes as a function of the observed band, and is determined by the temperature at which the peak of the black body spectrum roughly coincides with the center of the filter transmission curve. This result is consistent with recent theoret- ical models and is different than the common statment that the plateau phase starts once hydrogen recombinations becomes important. The temperatures we find agree with the predicted values for typical RSG progenitors of type-II SNe (Shussman et al. 2016a).

- We find that the temperature evolves with time as a power law, which flattens at $\sim 6000-8000 \mathrm{~K}$. We observe a similar evolution in the bolometric luminosity, where the logarithmic derivative at early phases is higher than that at late phases. The flattening is most likely a result of the recombination wave that exposes the inner layers. The values of the logarithmic derivatives for $\mathrm{T}$ and $\mathrm{L}_{\mathrm{bol}}$ at early phases agree with predictions from simulations and analytic works (Shussman et al. 2016a; Nakar \& Sari 2010).

- SN spectra deviate from a standard black body, both at low temperatures and short wavelengths due to line blanketing, and also at high temperatures and long wavelengths. We show that the SNe in our sample follow the analytic result from (Shussman et al. 2016a), that the flux on the RJ tail follows $F_{v} \propto v^{1.4}$. 


\begin{tabular}{lcccc}
\hline SN name & $\alpha_{\text {T,early }}$ & $\alpha_{\text {T,late }}$ & $\alpha_{\text {L,early }}$ & $\alpha_{\text {L, late }}$ \\
\hline SN1999em & $-0.34(0.06)_{-0.16}^{+0.18}$ & $-0.05(0.04)_{-0.00}^{+0.00}$ & $-0.54(0.09)_{-0.25}^{+0.28}$ & $-0.06(0.06)_{-0.01}^{+0.01}$ \\
SN2004et & $-0.21(0.12)_{-0.01}^{+0.01}$ & $-0.11(0.05)_{-0.00}^{+0.00}$ & $-0.24(0.17)_{-0.01}^{+0.01}$ & $-0.24(0.09)_{-0.00}^{+0.00}$ \\
SN2005cs & $-0.47(0.03)_{-0.00}^{+0.00}$ & $-0.06(0.07)_{-0.00}^{+0.00}$ & $-0.68(0.05)_{-0.00}^{+0.00}$ & $0.13(0.10)_{-0.00}^{+0.00}$ \\
SN2006bp & $-0.27(0.01)_{-0.03}^{+0.03}$ & & $-0.04(0.01)_{-0.01}^{+0.01}$ & \\
SN2007od & $-0.10(0.27)_{-0.06}^{+0.06}$ & $-0.09(0.06)_{-0.01}^{+0.01}$ & $-0.22(0.29)_{-0.14}^{+0.14}$ & $-0.81(0.09)_{-0.12}^{+0.12}$ \\
SN2008in & $-0.28(0.10)_{-0.26}^{+0.28}$ & $-0.07(0.07)_{-0.01}^{+0.01}$ & $-0.50(0.12)_{-0.46}^{+0.49}$ & $-0.52(0.07)_{-0.09}^{+0.09}$ \\
SN2009N & $-0.48(0.22)_{-0.34}^{+0.35}$ & $-0.07(0.05)_{-0.01}^{+0.01}$ & $-0.21(0.19)_{-0.15}^{+0.15}$ & $0.11(0.05)_{-0.02}^{+0.02}$ \\
SN2009bw & $-0.67(0.05)_{-0.21}^{+0.23}$ & $-0.04(0.19)_{-0.00}^{+0.00}$ & $-0.79(0.07)_{-0.24}^{+0.25}$ & $-0.29(0.10)_{-0.01}^{+0.01}$ \\
SN2009ib & $-0.29(0.12)_{-0.15}^{+0.16}$ & $-0.14(0.08)_{-0.02}^{+0.02}$ & $-0.32(0.15)_{-0.17}^{+0.18}$ & $-0.03(0.05)_{-0.00}^{+0.00}$ \\
SN2012A & $-0.84(0.11)_{-0.37}^{+0.38}$ & $-0.09(0.06)_{-0.01}^{+0.01}$ & $-1.07(0.18)_{-0.47}^{+0.48}$ & $-0.87(0.07)_{-0.06}^{+0.06}$ \\
SN2012aw & $-0.35(0.02)_{-0.02}^{+0.02}$ & $-0.11(0.04)_{-0.00}^{+0.00}$ & $-0.33(0.03)_{-0.02}^{+0.02}$ & $-0.09(0.06)_{-0.00}^{+0.00}$ \\
SN2012ec & $-0.50(0.21)_{-0.38}^{+0.39}$ & $-0.11(0.07)_{-0.02}^{+0.02}$ & $-0.75(0.22)_{-0.56}^{+0.58}$ & $-0.06(0.13)_{-0.01}^{+0.01}$ \\
SN2013ab & $-0.56(0.02)_{-0.07}^{+0.07}$ & & $-0.80(0.03)_{-0.10}^{+0.10}$ & \\
SN2013by & $-0.30(0.01)_{-0.84}^{+0.38}$ & & $-0.42(0.01)_{-1.03}^{+0.49}$ & \\
SN2013ej & $-0.21(0.03)_{-0.03}^{+0.04}$ & & $-0.00(0.05)_{-0.00}^{+0.00}$ & \\
\hline
\end{tabular}

Table 3. The early and late logarithmic derivatives of the temperature and the bolometric luminosity. The values of $\alpha_{\mathrm{T}, \text { early }}$ and $\alpha_{\mathrm{L}, \text { early }}$ increase by $\approx 0.1$ and $\approx 0.2$ respectively, with an extinction value of $\mathrm{E}(\mathrm{B}-\mathrm{V})=0.1$ mag.

\section{ACKNOWLEDGEMENTS}

This research was supported by the I-Core center of excellence of the CHE-ISF. TF and EN were partially supported by an ERC starting grant (GRB/SN), an ISF grant $(1277 / 13)$ and an ISA grant. 

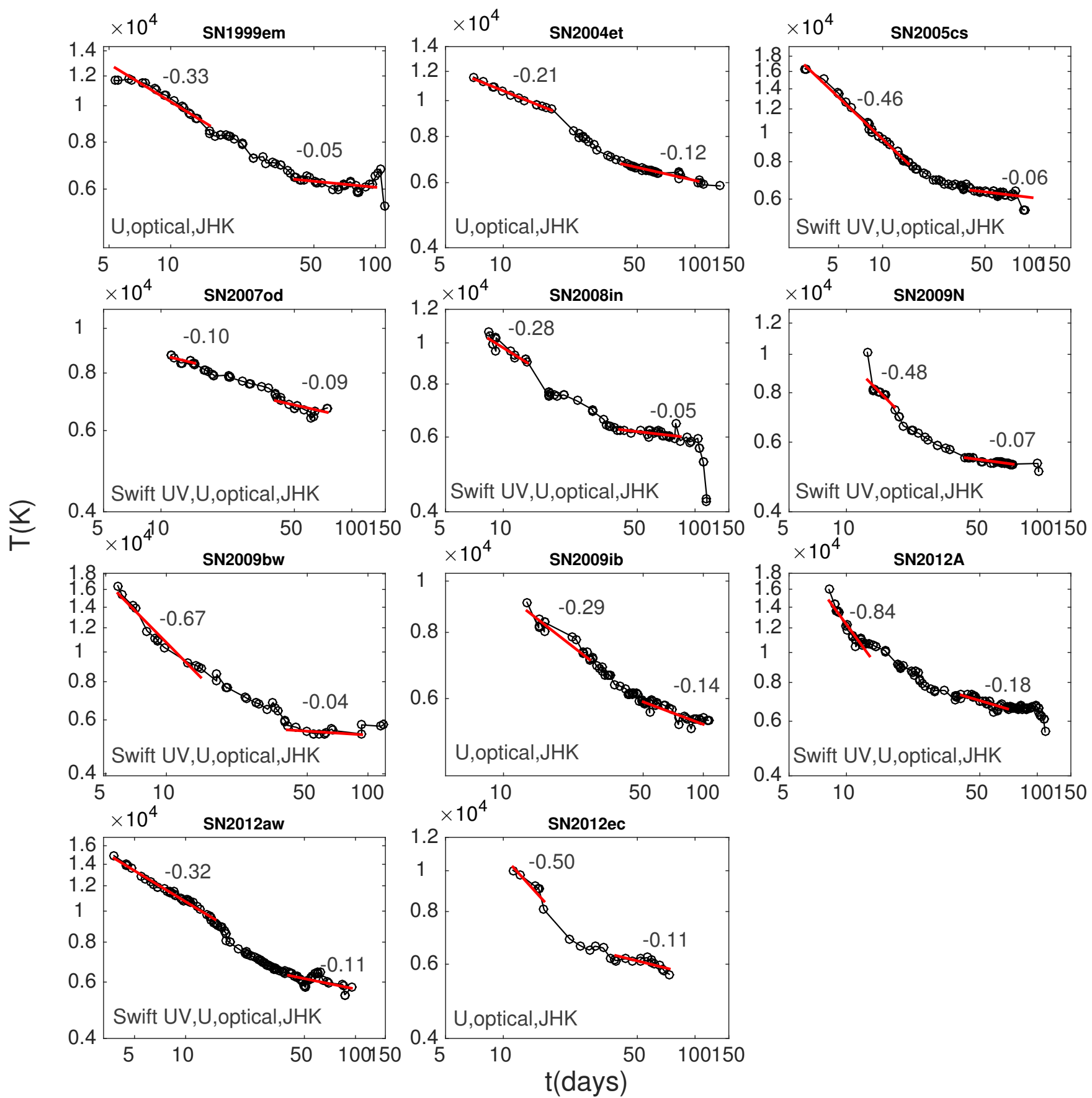

Figure 11. Temperature curves of objects that have both UV and JHK data. The numbers indicate the values of the best-fit early and late logarithmic derivative computed during the first 15 days, and during days 40-100 after the explosion, respectively. A clear flattening of the temperature is observed as the SN approcahes the temperaure of hydrogen recombination. 

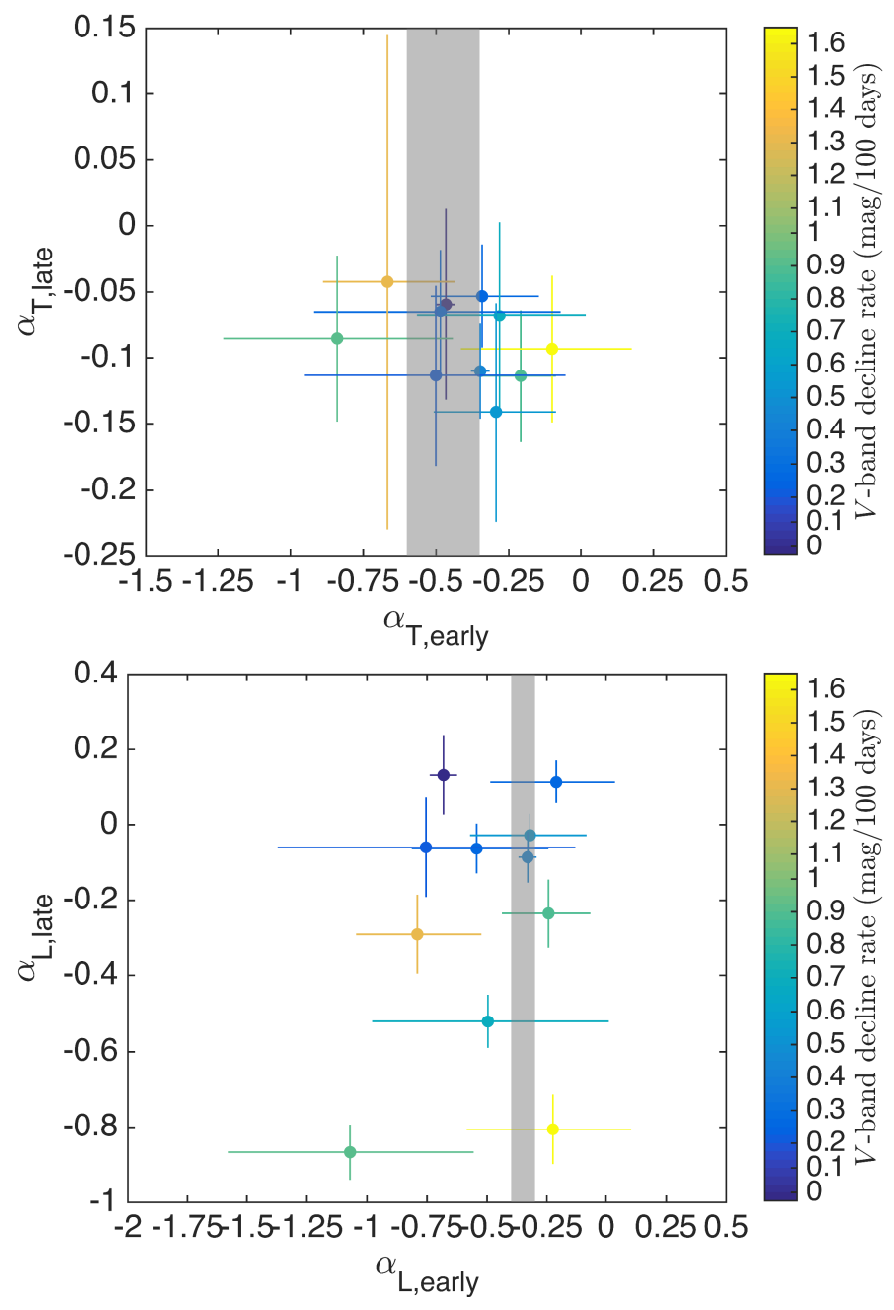

Figure 12. Top: the logarithmic derivative of the temperature at late times (40-100 days after the explosion) vs. the logarithmic derivative of the temperature at early times (up to 15 days after the explosion). The colors represent the decline rate of the $V$-band light curve. The values of the different objects agree with each other within the error-bars, and there is no apparent correlation with the light curve decline rate. Bottom: the same as the top figure for the bolometric luminosity. In this case, the early values of the logarithmic derivatives agree within the errors, but the late values show a wider spread. SNe whose luminosity declines faster at late phases (i.e., during the plateau phase) have faster declining light curves. 

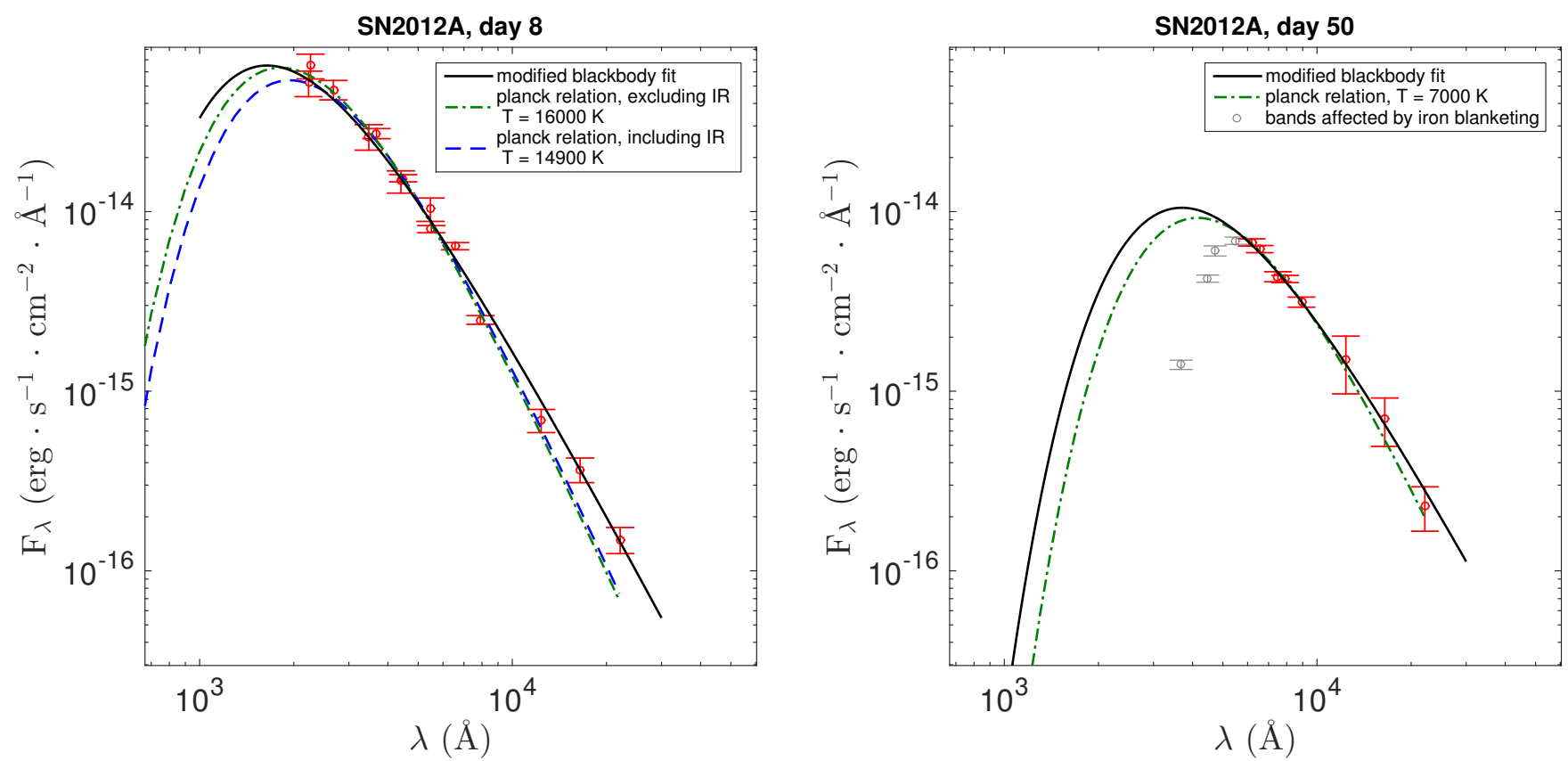

Figure 13. left:The SED of SN2012A at 8 days after the explosion. The standard Planck formula is only able to fit the peak of the distribution, but fails to fit the RJ tail. A modified black body model from Shussman et al. (2016a) is shown to be compatible throughout the whole wavelength range. right: An SED of SN2012A at 50 days past explosion. At this stage, after the onset of recombination and at low temperatures, both the modified black body and the standard model are able to describe the data. 


\section{REFERENCES}

Anderson J. P., et al., 2014, ApJ, 786, 67

Arcavi I., et al., 2012, ApJ, 756, L30

Arnett W. D., 1980, ApJ, 237, 541

Barbarino C., et al., 2015, MNRAS, 448, 2312

Bersten M. C., Hamuy M., 2009, ApJ, 701, 200

Bose S., et al., 2013, MNRAS, 433, 1871

Bose S., et al., 2015a, MNRAS, 450, 2373

Bose S., et al., 2015b, ApJ, 806, 160

Cardelli J. A., Clayton G. C., Mathis J. S., 1989, ApJ, 345, 245

Dall'Ora M., et al., 2014, ApJ, 787, 139

Eastman R. G., Schmidt B. P., Kirshner R., 1996, ApJ, 466, 911

Falk S. W., Arnett W. D., 1977, ApJS, 33, 515

Faran T., et al., 2014a, MNRAS, 442, 844

Faran T., et al., 2014b, MNRAS, 445, 554

Fraser M., et al., 2011, MNRAS, 417, 1417

Gurugubelli U. K., Sahu D. K., Anupama G. C., Chakradhari N. K., 2008, Bulletin of the Astronomical Society of India, 36,79

Hendry M. A., et al., 2006, MNRAS, 369, 1303

Inserra C., et al., 2011, MNRAS, 417, 261

Inserra C., et al., 2012, MNRAS, 422, 1122

Kasen D., Woosley S. E., 2009, ApJ, 703, 2205

Leonard D. C., et al., 2002a, PASP, 114, 35

Leonard D. C., et al., 2002b, AJ, 124, 2490

Lusk J. A., 2016, SuperBoL: Module for calculating the bolometric luminosities of supernovae, Astrophysics Source Code Library (ascl:1609.019)

Maguire K., et al., 2010, MNRAS, 404, 981

Nakar E., Sari R., 2010, ApJ, 725, 904

Nakar E., Poznanski D., Katz B., 2016, ApJ, 823, 127

Pastorello A., et al., 2009, MNRAS, 394, 2266

Patat F., Barbon R., Cappellaro E., Turatto M., 1994, A\&A, 282, 731

Pejcha O., Prieto J. L., 2015, ApJ, 799, 215

Pinto P. A., Eastman R. G., 2000, ApJ, 530, 757

Quimby R. M., Wheeler J. C., Höflich P., Akerlof C. W., Brown P. J., Rykoff E. S., 2007, ApJ, 666, 1093

Rabinak I., Waxman E., 2011, ApJ, 728, 63

Richmond M. W., 2014, Journal of the American Association of Variable Star Observers (JAAVSO), 42, 333

Roy R., Kumar B., 2012, in Astronomical Society of India Conference Series. p. 115

Shussman T., Waldman R., Nakar E., 2016a, preprint, (arXiv: 1610.05323)

Shussman T., Nakar E., Waldman R., Katz B., 2016b, preprint, (arXiv: 1602.02774)

Smartt S. J., 2015, Publ. Astron. Soc. Australia, 32, e016

Takáts K., et al., 2014, MNRAS, 438, 368

Takáts K., et al., 2015, MNRAS, 450, 3137

Tomasella L., et al., 2013, MNRAS, 434, 1636

Tominaga N., Morokuma T., Blinnikov S. I., Baklanov P., Sorokina E. I., Nomoto K., 2011, ApJS, 193, 20

Utrobin V. P., 2007, A\&A, 461, 233

Valenti S., et al., 2014, MNRAS, 438, L101

Valenti S., et al., 2015, MNRAS, 448, 2608

Valenti S., et al., 2016, MNRAS, 459, 3939

Van Dyk S. D., Li W., Filippenko A. V., 2003a, PASP, 115, 448

Van Dyk S. D., Li W., Filippenko A. V., 2003b, PASP, 115, 1289

Van Dyk S. D., et al., 2012, ApJ, 756, 131

Young T. R., 2004, ApJ, 617, 1233

\section{APPENDIX A: A LIST OF THE TEMPERATURES AND BOLOMETRIC LUMINOSITIES}

This paper has been typeset from a $\mathrm{T}_{\mathrm{E}} \mathrm{X} / \mathrm{LAT}_{\mathrm{E}} \mathrm{X}$ file prepared by the author. 
Table A1. A List of the Temperatures and Bolometric Luminosities

\begin{tabular}{|c|c|c|c|}
\hline MJD & $t-t_{\text {explosion }}$ & $\mathrm{T}(\mathrm{K})$ & $\mathrm{L}\left(10^{42} \mathrm{erg} \cdot \mathrm{s}^{-1}\right)$ \\
\hline \multicolumn{4}{|c|}{ SN1999em } \\
\hline 51481 & 5 & $11670_{761}^{904}$ & $1.43_{0.14}^{0.19}$ \\
\hline 51482 & 6 & $11748_{707}^{841}$ & $1.53_{0.15}^{0.17}$ \\
\hline 51483 & 7 & $11493_{597}^{652}$ & $1.50_{0.12}^{0.14}$ \\
\hline 51484 & 8 & $11075_{532}^{632}$ & $1.42_{0.10}^{0.13}$ \\
\hline 51485 & 9 & $10681_{529}^{576}$ & $1.35_{0.09}^{0.11}$ \\
\hline 51486 & 10 & $10320_{441}^{506}$ & $1.27_{0.07}^{0.09}$ \\
\hline 51487 & 11 & $9944_{432}^{527}$ & $1.19_{0.07}^{0.09}$ \\
\hline 51488 & 12 & $9539_{381}^{415}$ & $1.13_{0.05}^{0.06}$ \\
\hline 51489 & 13 & $9250_{336}^{379}$ & $1.08_{0.05}^{0.05}$ \\
\hline 51491 & 15 & $8481_{236}^{356}$ & $1.00_{0.04}^{0.04}$ \\
\hline 51492 & 16 & $8300_{251}^{272}$ & $0.97_{0.06}^{0.06}$ \\
\hline 51493 & 17 & $8340_{220}^{253}$ & $0.99_{0.05}^{0.06}$ \\
\hline 51494 & 18 & $8340_{237}^{234}$ & $1.00_{0.05}^{0.05}$ \\
\hline 51495 & 19 & $8310_{239}^{245}$ & $0.99_{0.05}^{0.05}$ \\
\hline 51496 & 20 & $8160_{228}^{231}$ & $0.96_{0.05}^{0.05}$ \\
\hline 51498 & 22 & $7899_{255}^{260}$ & $0.90_{0.05}^{0.05}$ \\
\hline 51501 & 25 & $7250_{181}^{176}$ & $0.86_{0.04}^{0.04}$ \\
\hline 51504 & 28 & $7320_{178}^{174}$ & $0.85_{0.03}^{0.04}$ \\
\hline 51505 & 29 & $7040_{161}^{162}$ & $0.83_{0.03}^{0.03}$ \\
\hline 51507 & 31 & $7060_{162}^{162}$ & $0.83_{0.03}^{0.03}$ \\
\hline 51508 & 32 & $7040_{158}^{165}$ & $0.83_{0.03}^{0.03}$ \\
\hline 51510 & 34 & $6950_{166}^{173}$ & $0.81_{0.03}^{0.03}$ \\
\hline 51513 & 37 & $6740_{144}^{149}$ & $0.80_{0.03}^{0.03}$ \\
\hline 51514 & 38 & $6600_{141}^{138}$ & $0.81_{0.03}^{0.03}$ \\
\hline 51516 & 40 & $6440_{131}^{137}$ & $0.82_{0.03}^{0.03}$ \\
\hline 51518 & 42 & $6340_{131}^{124}$ & $0.80_{0.03}^{0.03}$ \\
\hline 51519 & 43 & $6320_{124}^{130}$ & $0.80_{0.02}^{0.03}$ \\
\hline 51520 & 44 & $6360_{120}^{139}$ & $0.80_{0.02}^{0.03}$ \\
\hline 51522 & 46 & $6480_{132}^{136}$ & $0.82_{0.03}^{0.03}$ \\
\hline 51523 & 47 & $6460_{132}^{135}$ & $0.83_{0.03}^{0.03}$ \\
\hline 51526 & 50 & $6300_{121}^{132}$ & $0.82_{0.03}^{0.03}$ \\
\hline 51527 & 51 & $6260_{124}^{125}$ & $0.81_{0.03}^{0.03}$ \\
\hline 51528 & 52 & $6260_{113}^{138}$ & $0.81_{0.02}^{0.03}$ \\
\hline 51530 & 54 & $6220_{119}^{126}$ & $0.81_{0.02}^{0.03}$ \\
\hline 51538 & 62 & $6000_{112}^{115}$ & $0.80_{0.02}^{0.02}$ \\
\hline 51541 & 65 & $6033_{137}^{224}$ & $0.80_{0.03}^{0.05}$ \\
\hline 51546 & 70 & $6150_{168}^{179}$ & $0.83_{0.04}^{0.04}$ \\
\hline 51547 & 71 & $6140_{165}^{172}$ & $0.83_{0.04}^{0.04}$ \\
\hline 51551 & 75 & $6270_{172}^{187}$ & $0.83_{0.04}^{0.04}$ \\
\hline 51556 & 80 & $6120_{161}^{165}$ & $0.81_{0.04}^{0.04}$ \\
\hline 51557 & 81 & $5920_{277}^{312}$ & $0.76_{0.07}^{0.08}$ \\
\hline
\end{tabular}


Table A1 - continued A List of the Temperatures and Bolometric Luminosities

\begin{tabular}{|c|c|c|c|}
\hline 51558 & 82 & $5891_{324}^{341}$ & $0.76_{0.08}^{0.09}$ \\
\hline 51565 & 89 & $6080_{169}^{166}$ & $0.77_{0.04}^{0.04}$ \\
\hline 51570 & 94 & $6200_{172}^{164}$ & $0.76_{0.04}^{0.03}$ \\
\hline 51572 & 96 & $6200_{171}^{165}$ & $0.74_{0.03}^{0.03}$ \\
\hline 51576 & 100 & $6480_{183}^{188}$ & $0.72_{0.03}^{0.04}$ \\
\hline 51578 & 102 & $6600_{193}^{193}$ & $0.71_{0.04}^{0.04}$ \\
\hline 51581 & 105 & $6780_{266}^{280}$ & $0.69_{0.04}^{0.04}$ \\
\hline 51586 & 110 & $5400_{449}^{538}$ & $0.40_{0.06}^{0.08}$ \\
\hline 51592 & 116 & $4960_{367}^{447}$ & $0.29_{0.04}^{0.05}$ \\
\hline 51599 & 123 & $4960_{376}^{435}$ & $0.21_{0.03}^{0.04}$ \\
\hline 51604 & 128 & $5500_{247}^{292}$ & $0.17_{0.01}^{0.02}$ \\
\hline 51606 & 130 & $5620_{230}^{233}$ & $0.16_{0.01}^{0.01}$ \\
\hline 51607 & 131 & $5620_{144}^{163}$ & $0.16_{0.01}^{0.01}$ \\
\hline 51613 & 137 & $5640_{162}^{154}$ & $0.14_{0.01}^{0.01}$ \\
\hline 51614 & 138 & $5640_{135}^{161}$ & $0.14_{0.01}^{0.01}$ \\
\hline 51619 & 143 & $5760_{142}^{156}$ & $0.14_{0.01}^{0.01}$ \\
\hline 51620 & 144 & $5760_{147}^{139}$ & $0.14_{0.01}^{0.01}$ \\
\hline 51624 & 148 & $5920_{152}^{151}$ & $0.15_{0.01}^{0.01}$ \\
\hline 51627 & 151 & $5900_{144}^{158}$ & $0.14_{0.01}^{0.01}$ \\
\hline 51629 & 153 & $5940_{155}^{150}$ & $0.14_{0.01}^{0.01}$ \\
\hline 51634 & 158 & $5960_{150}^{159}$ & $0.13_{0.01}^{0.01}$ \\
\hline 51637 & 161 & $5880_{165}^{158}$ & $0.12_{0.01}^{0.01}$ \\
\hline 51638 & 162 & $5160_{454}^{597}$ & $0.10_{0.01}^{0.02}$ \\
\hline 51639 & 163 & $5460_{546}^{707}$ & $0.11_{0.02}^{0.03}$ \\
\hline 51640 & 164 & $5300_{516}^{680}$ & $0.10_{0.02}^{0.03}$ \\
\hline 51641 & 165 & $5024_{518}^{1549}$ & $0.09_{0.01}^{0.06}$ \\
\hline 51642 & 166 & $5640_{602}^{774}$ & $0.11_{0.02}^{0.03}$ \\
\hline 51643 & 167 & $5880_{531}^{671}$ & $0.11_{0.02}^{0.03}$ \\
\hline 51644 & 168 & $5920_{561}^{751}$ & $0.12_{0.02}^{0.03}$ \\
\hline 51650 & 174 & $5580_{472}^{595}$ & $0.09_{0.01}^{0.02}$ \\
\hline 51653 & 177 & $5400_{450}^{534}$ & $0.08_{0.01}^{0.02}$ \\
\hline 51655 & 179 & $6080_{571}^{727}$ & $0.10_{0.02}^{0.03}$ \\
\hline 51656 & 180 & $5560_{475}^{580}$ & $0.08_{0.01}^{0.02}$ \\
\hline \multicolumn{4}{|c|}{ SN1999gi } \\
\hline 51524 & 5 & $8680_{611}^{752}$ & $0.57_{0.05}^{0.07}$ \\
\hline 51525 & 6 & $8480_{323}^{347}$ & $0.59_{0.02}^{0.03}$ \\
\hline 51528 & 9 & $8100_{298}^{307}$ & $0.65_{0.02}^{0.02}$ \\
\hline 51529 & 10 & $7880_{280}^{291}$ & $0.63_{0.02}^{0.02}$ \\
\hline 51530 & 11 & $7720_{269}^{276}$ & $0.63_{0.02}^{0.02}$ \\
\hline 51531 & 12 & $7560_{253}^{269}$ & $0.62_{0.02}^{0.02}$ \\
\hline 51534 & 15 & $7436_{307}^{700}$ & $0.60_{0.02}^{0.06}$ \\
\hline 51540 & 21 & $6860_{389}^{411}$ & $0.59_{0.02}^{0.03}$ \\
\hline 51545 & 26 & $6760_{360}^{419}$ & $0.56_{0.02}^{0.03}$ \\
\hline
\end{tabular}


Faran et al.

Table A1 - continued A List of the Temperatures and Bolometric Luminosities

\begin{tabular}{|c|c|c|c|}
\hline 51550 & 31 & $6120_{292}^{334}$ & $0.52_{0.02}^{0.02}$ \\
\hline 51556 & 37 & $5920_{274}^{308}$ & $0.50_{0.01}^{0.01}$ \\
\hline 51577 & 58 & $5320_{219}^{243}$ & $0.50_{0.01}^{0.01}$ \\
\hline 51582 & 63 & $5260_{219}^{232}$ & $0.49_{0.01}^{0.01}$ \\
\hline 51590 & 71 & $5080_{197}^{223}$ & $0.50_{0.02}^{0.02}$ \\
\hline 51614 & 95 & $5140_{211}^{218}$ & $0.45_{0.01}^{0.02}$ \\
\hline 51619 & 100 & $4960_{198}^{200}$ & $0.45_{0.01}^{0.02}$ \\
\hline 51624 & 105 & $4880_{181}^{205}$ & $0.43_{0.01}^{0.02}$ \\
\hline 51629 & 110 & $4880_{185}^{200}$ & $0.40_{0.01}^{0.01}$ \\
\hline 51634 & 115 & $4700_{177}^{178}$ & $0.36_{0.01}^{0.02}$ \\
\hline 51638 & 119 & $4580_{169}^{167}$ & $0.31_{0.01}^{0.01}$ \\
\hline 51643 & 124 & $4220_{139}^{145}$ & $0.25_{0.01}^{0.01}$ \\
\hline 51648 & 129 & $4060_{185}^{211}$ & $0.17_{0.01}^{0.02}$ \\
\hline 51653 & 134 & $3760_{110}^{116}$ & $0.13_{0.01}^{0.01}$ \\
\hline 51658 & 139 & $3300_{142}^{155}$ & $0.17_{0.02}^{0.03}$ \\
\hline 51664 & 145 & $3640_{107}^{104}$ & $0.11_{0.01}^{0.01}$ \\
\hline 51669 & 150 & $3620_{108}^{101}$ & $0.11_{0.01}^{0.01}$ \\
\hline \multicolumn{4}{|c|}{ SN2000dc } \\
\hline 51766 & 4 & $8540_{327}^{353}$ & $1.39_{0.06}^{0.06}$ \\
\hline 51767 & 5 & $8220_{300}^{327}$ & $1.46_{0.05}^{0.06}$ \\
\hline 51773 & 11 & $7100_{215}^{243}$ & $1.70_{0.04}^{0.05}$ \\
\hline 51775 & 13 & $7020_{214}^{231}$ & $1.69_{0.04}^{0.05}$ \\
\hline 51777 & 15 & $6940_{208}^{226}$ & $1.63_{0.04}^{0.04}$ \\
\hline 51779 & 17 & $6800_{210}^{206}$ & $1.55_{0.04}^{0.04}$ \\
\hline 51781 & 19 & $6540_{187}^{196}$ & $1.50_{0.04}^{0.03}$ \\
\hline \multicolumn{4}{|c|}{ SN2001cm } \\
\hline 52069 & 5 & $9580_{424}^{494}$ & $1.63_{0.09}^{0.11}$ \\
\hline 52070 & 6 & $9840_{455}^{516}$ & $1.73_{0.10}^{0.12}$ \\
\hline 52071 & 7 & $9960_{467}^{531}$ & $1.78_{0.10}^{0.13}$ \\
\hline 52072 & 8 & $9780_{451}^{507}$ & $1.73_{0.10}^{0.11}$ \\
\hline 52077 & 13 & $9280_{413}^{438}$ & $1.54_{0.08}^{0.09}$ \\
\hline 52082 & 18 & $8300_{329}^{370}$ & $1.31_{0.05}^{0.06}$ \\
\hline 52087 & 23 & $7440_{273}^{303}$ & $1.13_{0.04}^{0.04}$ \\
\hline \multicolumn{4}{|c|}{ SN2001cy } \\
\hline 52092 & 6 & $11400_{605}^{687}$ & $3.32_{0.27}^{0.33}$ \\
\hline 52093 & 7 & $11140_{587}^{638}$ & $3.17_{0.24}^{0.29}$ \\
\hline 52094 & 8 & $10920_{557}^{615}$ & $3.05_{0.22}^{0.27}$ \\
\hline 52095 & 9 & $10720_{516}^{614}$ & $2.95_{0.20}^{0.26}$ \\
\hline 52096 & 10 & $10620_{668}^{759}$ & $2.89_{0.27}^{0.34}$ \\
\hline 52097 & 11 & $10480_{519}^{548}$ & $2.81_{0.19}^{0.22}$ \\
\hline 52098 & 12 & $10200_{886}^{1115}$ & $2.68_{0.26}^{0.39}$ \\
\hline 52099 & 13 & $10240_{480}^{535}$ & $2.66_{0.17}^{0.20}$ \\
\hline
\end{tabular}


Table A1 - continued A List of the Temperatures and Bolometric Luminosities

\begin{tabular}{|c|c|c|c|}
\hline 52100 & 14 & $9878_{522}^{1460}$ & $2.48_{0.18}^{0.63}$ \\
\hline 52102 & 16 & $10020_{853}^{1028}$ & $2.49_{0.30}^{0.42}$ \\
\hline 52104 & 18 & $9680_{787}^{954}$ & $2.30_{0.26}^{0.36}$ \\
\hline 52106 & 20 & $9460_{812}^{1017}$ & $2.17_{0.25}^{0.37}$ \\
\hline 52112 & 26 & $8400_{568}^{699}$ & $1.65_{0.12}^{0.18}$ \\
\hline 52117 & 31 & $7740_{495}^{553}$ & $1.38_{0.09}^{0.11}$ \\
\hline 52122 & 36 & $7260_{422}^{500}$ & $1.22_{0.06}^{0.08}$ \\
\hline 52132 & 46 & $6660_{356}^{402}$ & $1.06_{0.04}^{0.05}$ \\
\hline 52137 & 51 & $6540_{336}^{389}$ & $1.02_{0.03}^{0.04}$ \\
\hline 52142 & 56 & $6440_{322}^{379}$ & $0.98_{0.03}^{0.04}$ \\
\hline 52147 & 61 & $6380_{326}^{357}$ & $0.94_{0.03}^{0.04}$ \\
\hline 52152 & 66 & $6220_{309}^{338}$ & $0.90_{0.03}^{0.03}$ \\
\hline 52157 & 71 & $6080_{290}^{327}$ & $0.88_{0.03}^{0.03}$ \\
\hline 52162 & 76 & $5940_{273}^{323}$ & $0.87_{0.02}^{0.03}$ \\
\hline 52167 & 81 & $5980_{276}^{320}$ & $0.86_{0.02}^{0.03}$ \\
\hline 52172 & 86 & $6060_{294}^{318}$ & $0.83_{0.02}^{0.03}$ \\
\hline 52177 & 91 & $5820_{269}^{291}$ & $0.77_{0.02}^{0.02}$ \\
\hline 52182 & 96 & $5600_{242}^{276}$ & $0.71_{0.02}^{0.02}$ \\
\hline \multicolumn{4}{|c|}{ SN2001do } \\
\hline 52137 & 3 & $8780_{339}^{385}$ & $1.34_{0.06}^{0.07}$ \\
\hline 52138 & 4 & $8117_{362}^{1077}$ & $1.29_{0.05}^{0.22}$ \\
\hline 52139 & 5 & $8300_{551}^{682}$ & $1.42_{0.10}^{0.15}$ \\
\hline 52140 & 6 & $7780_{490}^{574}$ & $1.36_{0.08}^{0.11}$ \\
\hline 52141 & 7 & $7680_{486}^{544}$ & $1.36_{0.08}^{0.10}$ \\
\hline 52142 & 8 & $7500_{462}^{514}$ & $1.35_{0.08}^{0.09}$ \\
\hline 52145 & 11 & $7100_{412}^{452}$ & $1.34_{0.06}^{0.08}$ \\
\hline 52148 & 14 & $6580_{345}^{387}$ & $1.28_{0.05}^{0.05}$ \\
\hline 52151 & 17 & $6720_{359}^{409}$ & $1.19_{0.04}^{0.06}$ \\
\hline 52154 & 20 & $6360_{312}^{370}$ & $1.14_{0.04}^{0.04}$ \\
\hline 52157 & 23 & $6240_{318}^{332}$ & $1.07_{0.03}^{0.04}$ \\
\hline 52160 & 26 & $6200_{298}^{347}$ & $1.03_{0.03}^{0.03}$ \\
\hline 52163 & 29 & $5900_{274}^{303}$ & $0.99_{0.03}^{0.03}$ \\
\hline 52166 & 32 & $5600_{245}^{271}$ & $0.93_{0.03}^{0.02}$ \\
\hline 52169 & 35 & $5640_{251}^{273}$ & $0.87_{0.02}^{0.02}$ \\
\hline 52172 & 38 & $5520_{241}^{258}$ & $0.81_{0.02}^{0.02}$ \\
\hline 52175 & 41 & $5340_{227}^{239}$ & $0.77_{0.02}^{0.02}$ \\
\hline 52181 & 47 & $5120_{208}^{218}$ & $0.69_{0.02}^{0.02}$ \\
\hline 52184 & 50 & $5160_{206}^{228}$ & $0.67_{0.02}^{0.02}$ \\
\hline 52187 & 53 & $4860_{174}^{210}$ & $0.65_{0.02}^{0.02}$ \\
\hline 52190 & 56 & $4940_{192}^{202}$ & $0.60_{0.02}^{0.02}$ \\
\hline 52193 & 59 & $4960_{200}^{197}$ & $0.58_{0.02}^{0.02}$ \\
\hline 52198 & 64 & $4760_{168}^{199}$ & $0.57_{0.02}^{0.02}$ \\
\hline 52203 & 69 & $4000_{239}^{254}$ & $0.69_{0.08}^{0.10}$ \\
\hline
\end{tabular}


Faran et al.

Table A1 - continued A List of the Temperatures and Bolometric Luminosities

\begin{tabular}{|c|c|c|c|}
\hline \multicolumn{4}{|c|}{ SN2001fa } \\
\hline 52202 & 4 & $14820_{1073}^{1262}$ & $12.54_{1.66}^{2.21}$ \\
\hline 52203 & 5 & $13500_{879}^{1011}$ & $11.47_{1.29}^{1.65}$ \\
\hline 52204 & 6 & $11980_{671}^{774}$ & $9.75_{0.86}^{1.09}$ \\
\hline 52205 & 7 & $11320_{591}^{682}$ & $9.22_{0.72}^{0.90}$ \\
\hline 52206 & 8 & $10580_{510}^{583}$ & $8.46_{0.56}^{0.70}$ \\
\hline 52207 & 9 & $9800_{441}^{479}$ & $7.38_{0.42}^{0.49}$ \\
\hline 52208 & 10 & $9560_{414}^{458}$ & $6.97_{0.37}^{0.44}$ \\
\hline 52209 & 11 & $9160_{377}^{416}$ & $6.38_{0.31}^{0.35}$ \\
\hline 52217 & 19 & $7600_{246}^{283}$ & $3.72_{0.11}^{0.12}$ \\
\hline 52218 & 20 & $7613_{419}^{609}$ & $3.30_{0.17}^{0.28}$ \\
\hline 52219 & 21 & $7760_{662}^{788}$ & $3.20_{0.21}^{0.32}$ \\
\hline 52221 & 23 & $7580_{461}^{542}$ & $2.98_{0.17}^{0.22}$ \\
\hline 52223 & 25 & $7600_{474}^{532}$ & $2.67_{0.15}^{0.20}$ \\
\hline 52228 & 30 & $7380_{438}^{507}$ & $2.33_{0.12}^{0.16}$ \\
\hline 52231 & 33 & $6980_{399}^{432}$ & $2.20_{0.10}^{0.12}$ \\
\hline 52250 & 52 & $6900_{376}^{440}$ & $1.85_{0.08}^{0.10}$ \\
\hline 52278 & 80 & $5960_{407}^{478}$ & $0.53_{0.02}^{0.03}$ \\
\hline 52283 & 85 & $5740_{735}^{1013}$ & $0.48_{0.04}^{0.05}$ \\
\hline 52288 & 90 & $6380_{920}^{1337}$ & $0.46_{0.05}^{0.10}$ \\
\hline 52293 & 95 & $5200_{597}^{811}$ & $0.33_{0.02}^{0.02}$ \\
\hline \multicolumn{4}{|c|}{ SN2001x } \\
\hline 51976 & 13 & $10840_{533}^{624}$ & $1.76_{0.12}^{0.16}$ \\
\hline 51984 & 21 & $8669_{419}^{1259}$ & $1.30_{0.06}^{0.28}$ \\
\hline 51989 & 26 & $8800_{759}^{933}$ & $1.34_{0.15}^{0.21}$ \\
\hline 51995 & 32 & $7920_{512}^{594}$ & $1.17_{0.08}^{0.10}$ \\
\hline 52000 & 37 & $7500_{453}^{526}$ & $1.11_{0.06}^{0.08}$ \\
\hline 52005 & 42 & $7160_{411}^{473}$ & $1.08_{0.05}^{0.06}$ \\
\hline 52012 & 49 & $6900_{388}^{424}$ & $1.05_{0.04}^{0.05}$ \\
\hline 52017 & 54 & $6780_{363}^{421}$ & $1.04_{0.04}^{0.05}$ \\
\hline 52022 & 59 & $6700_{367}^{392}$ & $1.03_{0.04}^{0.05}$ \\
\hline 52027 & 64 & $6640_{346}^{402}$ & $1.00_{0.04}^{0.05}$ \\
\hline 52032 & 69 & $6580_{342}^{392}$ & $1.00_{0.03}^{0.04}$ \\
\hline 52040 & 77 & $6460_{338}^{363}$ & $0.97_{0.03}^{0.04}$ \\
\hline 52045 & 82 & $6340_{328}^{345}$ & $0.95_{0.03}^{0.03}$ \\
\hline 52050 & 87 & $6220_{300}^{350}$ & $0.92_{0.03}^{0.03}$ \\
\hline 52055 & 92 & $6160_{295}^{340}$ & $0.88_{0.03}^{0.03}$ \\
\hline 52060 & 97 & $6080_{297}^{318}$ & $0.81_{0.02}^{0.02}$ \\
\hline 52068 & 105 & $5760_{255}^{295}$ & $0.66_{0.02}^{0.02}$ \\
\hline 52074 & 111 & $5400_{235}^{241}$ & $0.50_{0.01}^{0.01}$ \\
\hline 52081 & 118 & $4980_{197}^{205}$ & $0.34_{0.01}^{0.01}$ \\
\hline 52089 & 126 & $4620_{167}^{187}$ & $0.24_{0.01}^{0.01}$ \\
\hline 52096 & 133 & $4760_{222}^{253}$ & $0.20_{0.01}^{0.01}$ \\
\hline
\end{tabular}


Table A1 - continued A List of the Temperatures and Bolometric Luminosities

\begin{tabular}{|c|c|c|c|}
\hline 52103 & 140 & $4500_{161}^{164}$ & $0.19_{0.01}^{0.01}$ \\
\hline 52110 & 147 & $4580_{164}^{173}$ & $0.18_{0.01}^{0.01}$ \\
\hline \multicolumn{4}{|c|}{ SN2002gd } \\
\hline 52555 & 2 & $13120_{826}^{985}$ & $1.04_{0.11}^{0.15}$ \\
\hline 52556 & 3 & $12340_{733}^{839}$ & $1.02_{0.10}^{0.12}$ \\
\hline 52559 & 6 & $10080_{477}^{522}$ & $0.99_{0.06}^{0.07}$ \\
\hline 52562 & 9 & $8640_{378}^{441}$ & $0.77_{0.04}^{0.05}$ \\
\hline 52570 & 17 & $8580_{1118}^{1633}$ & $0.74_{0.12}^{0.23}$ \\
\hline 52573 & 20 & $8680_{1688}^{3030}$ & $0.73_{0.15}^{0.44}$ \\
\hline 52576 & 23 & $8280_{1339}^{2067}$ & $0.67_{0.11}^{0.26}$ \\
\hline 52579 & 26 & $7940_{983}^{1297}$ & $0.61_{0.09}^{0.15}$ \\
\hline 52582 & 29 & $7860_{957}^{1276}$ & $0.59_{0.08}^{0.14}$ \\
\hline 52585 & 32 & $7880_{962}^{1283}$ & $0.57_{0.08}^{0.13}$ \\
\hline 52590 & 37 & $8180_{2548}^{8162}$ & $0.61_{0.19}^{1.60}$ \\
\hline 52598 & 45 & $7420_{846}^{1121}$ & $0.56_{0.06}^{0.11}$ \\
\hline 52608 & 55 & $6840_{715}^{928}$ & $0.51_{0.04}^{0.08}$ \\
\hline 52613 & 60 & $6880_{855}^{1155}$ & $0.51_{0.05}^{0.09}$ \\
\hline 52618 & 65 & $6900_{955}^{1388}$ & $0.52_{0.05}^{0.11}$ \\
\hline 52634 & 81 & $6460_{648}^{795}$ & $0.43_{0.03}^{0.05}$ \\
\hline 52659 & 106 & $5680_{813}^{1163}$ & $0.22_{0.01}^{0.03}$ \\
\hline 52664 & 111 & $5300_{1041}^{1800}$ & $0.15_{0.01}^{0.04}$ \\
\hline \multicolumn{4}{|c|}{ SN2003hf } \\
\hline 52867 & 3 & $19000_{2165}^{2873}$ & $11.39_{2.54}^{4.17}$ \\
\hline 52868 & 4 & $22940_{3142}^{4454}$ & $21.50_{6.09}^{11.31}$ \\
\hline 52869 & 5 & $19820_{2205}^{2926}$ & $18.27_{4.11}^{6.68}$ \\
\hline 52870 & 6 & $15560_{1215}^{1442}$ & $12.45_{1.82}^{2.48}$ \\
\hline 52871 & 7 & $13960_{958}^{1121}$ & $11.19_{1.36}^{1.78}$ \\
\hline 52872 & 8 & $13120_{951}^{1154}$ & $10.75_{1.25}^{1.73}$ \\
\hline 52873 & 9 & $12180_{705}^{826}$ & $10.13_{0.95}^{1.20}$ \\
\hline 52874 & 10 & $11820_{735}^{859}$ & $10.05_{0.93}^{1.21}$ \\
\hline 52875 & 11 & $11380_{597}^{719}$ & $9.83_{0.78}^{1.02}$ \\
\hline 52876 & 12 & $11040_{583}^{639}$ & $9.61_{0.74}^{0.89}$ \\
\hline 52877 & 13 & $10660_{536}^{595}$ & $9.33_{0.66}^{0.79}$ \\
\hline 52879 & 15 & $10040_{470}^{519}$ & $8.54_{0.52}^{0.62}$ \\
\hline 52880 & 16 & $9740_{433}^{494}$ & $8.13_{0.46}^{0.55}$ \\
\hline 52881 & 17 & $9420_{405}^{454}$ & $7.67_{0.40}^{0.47}$ \\
\hline 52884 & 20 & $8641_{415}^{1025}$ & $6.51_{0.35}^{1.10}$ \\
\hline 52886 & 22 & $8480_{594}^{702}$ & $6.14_{0.50}^{0.66}$ \\
\hline 52888 & 24 & $7960_{522}^{601}$ & $5.41_{0.36}^{0.48}$ \\
\hline 52890 & 26 & $7620_{468}^{552}$ & $4.80_{0.28}^{0.37}$ \\
\hline 52892 & 28 & $7440_{506}^{612}$ & $4.29_{0.27}^{0.37}$ \\
\hline 52894 & 30 & $7440_{508}^{581}$ & $3.91_{0.22}^{0.29}$ \\
\hline 52896 & 32 & $7400_{462}^{522}$ & $3.58_{0.20}^{0.25}$ \\
\hline
\end{tabular}


24 Faran et al.

Table A1 - continued A List of the Temperatures and Bolometric Luminosities

\begin{tabular}{llll}
52898 & 34 & $6980_{394}^{444}$ & $3.25_{0.14}^{0.18}$ \\
52900 & 36 & $6700_{400}^{459}$ & $3.00_{0.12}^{0.15}$ \\
52902 & 38 & $6505_{560}^{965}$ & $2.78_{0.17}^{0.41}$ \\
52904 & 40 & $6660_{729}^{945}$ & $2.70_{0.21}^{0.38}$ \\
52906 & 42 & $6740_{765}^{1007}$ & $2.60_{0.22}^{0.39}$ \\
52910 & 46 & $6380_{671}^{896}$ & $2.40_{0.16}^{0.29}$ \\
52913 & 49 & $6220_{723}^{936}$ & $2.31_{0.16}^{0.28}$ \\
52919 & 55 & $6780_{865}^{1205}$ & $2.07_{0.21}^{0.40}$ \\
\hline
\end{tabular}

\section{SN2003hk}

\begin{tabular}{|c|c|c|c|}
\hline 52873 & 13 & $10500_{513}^{582}$ & $4.17_{0.28}^{0.35}$ \\
\hline 52875 & 15 & $9600_{419}^{479}$ & $3.80_{0.21}^{0.25}$ \\
\hline 52877 & 17 & $9360_{403}^{445}$ & $3.57_{0.19}^{0.21}$ \\
\hline 52880 & 20 & $8660_{341}^{374}$ & $3.11_{0.13}^{0.15}$ \\
\hline 52881 & 21 & $8680_{343}^{375}$ & $2.99_{0.12}^{0.15}$ \\
\hline 52883 & 23 & $8720_{347}^{378}$ & $2.77_{0.12}^{0.14}$ \\
\hline 52885 & 25 & $8507_{626}^{670}$ & $2.53_{0.21}^{0.26}$ \\
\hline 52887 & 27 & $8580_{608}^{725}$ & $2.43_{0.20}^{0.27}$ \\
\hline 52889 & 29 & $8280_{878}^{1191}$ & $2.29_{0.24}^{0.42}$ \\
\hline 52893 & 33 & $8560_{798}^{1025}$ & $2.22_{0.26}^{0.39}$ \\
\hline 52900 & 40 & $7620_{590}^{681}$ & $1.63_{0.13}^{0.18}$ \\
\hline 52903 & 43 & $7260_{433}^{482}$ & $1.50_{0.07}^{0.10}$ \\
\hline 52906 & 46 & $7140_{415}^{467}$ & $1.42_{0.07}^{0.08}$ \\
\hline 52910 & 50 & $6760_{362}^{422}$ & $1.30_{0.05}^{0.06}$ \\
\hline 52913 & 53 & $6480_{336}^{377}$ & $1.20_{0.04}^{0.05}$ \\
\hline 52916 & 56 & $6500_{330}^{390}$ & $1.16_{0.04}^{0.05}$ \\
\hline 52919 & 59 & $6480_{340}^{372}$ & $1.14_{0.04}^{0.05}$ \\
\hline 52922 & 62 & $6460_{391}^{470}$ & $1.14_{0.05}^{0.06}$ \\
\hline 52928 & 68 & $5440_{311}^{358}$ & $1.07_{0.04}^{0.04}$ \\
\hline 52931 & 71 & $5960_{293}^{320}$ & $1.00_{0.03}^{0.03}$ \\
\hline 52935 & 75 & $5840_{286}^{299}$ & $0.93_{0.03}^{0.03}$ \\
\hline 52943 & 83 & $5300_{294}^{331}$ & $0.79_{0.03}^{0.03}$ \\
\hline 52948 & 88 & $5760_{380}^{429}$ & $0.75_{0.03}^{0.03}$ \\
\hline
\end{tabular}

\begin{tabular}{llll}
\hline SN2003iq & & & \\
\hline 52922 & 2 & $12940_{819}^{932}$ & $1.84_{0.20}^{0.25}$ \\
52927 & 7 & $9620_{438}^{460}$ & $1.83_{0.10}^{0.12}$ \\
52930 & 10 & $9260_{392}^{437}$ & $1.72_{0.09}^{0.10}$ \\
52933 & 13 & $8700_{345}^{376}$ & $1.54_{0.07}^{0.07}$ \\
52936 & 16 & $8111_{563}^{601}$ & $1.45_{0.11}^{0.13}$ \\
52939 & 19 & $8080_{543}^{619}$ & $1.49_{0.11}^{0.14}$ \\
52942 & 22 & $7880_{503}^{598}$ & $1.45_{0.09}^{0.12}$ \\
52945 & 25 & $7680_{470}^{572}$ & $1.38_{0.08}^{0.11}$ \\
52948 & 28 & $7560_{463}^{541}$ & $1.32_{0.07}^{0.10}$ \\
52955 & 35 & $6680_{350}^{415}$ & $1.21_{0.04}^{0.06}$
\end{tabular}


Table A1 - continued A List of the Temperatures and Bolometric Luminosities

\begin{tabular}{llll}
52960 & 40 & $6280_{377}^{430}$ & $1.20_{0.04}^{0.04}$ \\
52963 & 43 & $6860_{372}^{437}$ & $1.19_{0.05}^{0.06}$ \\
52966 & 46 & $6520_{348}^{373}$ & $1.15_{0.04}^{0.05}$ \\
52969 & 49 & $6540_{332}^{398}$ & $1.13_{0.04}^{0.05}$ \\
52972 & 52 & $6240_{305}^{352}$ & $1.13_{0.03}^{0.04}$ \\
52977 & 57 & $5560_{284}^{329}$ & $1.15_{0.03}^{0.04}$ \\
52992 & 72 & $6000_{292}^{309}$ & $0.99_{0.03}^{0.03}$ \\
52996 & 76 & $5980_{276}^{324}$ & $0.94_{0.03}^{0.03}$ \\
53002 & 82 & $5980_{290}^{307}$ & $0.88_{0.02}^{0.03}$ \\
53017 & 97 & $5580_{802}^{11770}$ & $0.56_{0.04}^{0.06}$ \\
53023 & 103 & $5020_{216}^{232}$ & $0.38_{0.01}^{0.02}$ \\
53026 & 106 & $5040_{193}^{224}$ & $0.29_{0.01}^{0.01}$ \\
53042 & 122 & $5120_{461}^{555}$ & $0.20_{0.01}^{0.01}$ \\
53045 & 125 & $4620_{223}^{256}$ & $0.20_{0.01}^{0.01}$ \\
53048 & 128 & $4520_{203}^{206}$ & $0.20_{0.01}^{0.01}$ \\
\hline
\end{tabular}

\begin{tabular}{|c|c|c|c|}
\hline \multicolumn{4}{|c|}{ SN2003z } \\
\hline 52670 & 5 & $12020_{698}^{783}$ & $0.22_{0.02}^{0.03}$ \\
\hline 52671 & 6 & $12300_{729}^{831}$ & $0.24_{0.02}^{0.03}$ \\
\hline 52674 & 9 & $10320_{497}^{556}$ & $0.19_{0.01}^{0.01}$ \\
\hline 52676 & 11 & $9520_{414}^{467}$ & $0.17_{0.01}^{0.01}$ \\
\hline 52679 & 14 & $8740_{477}^{1802}$ & $0.15_{0.01}^{0.05}$ \\
\hline 52691 & 26 & $7200_{606}^{755}$ & $0.12_{0.01}^{0.01}$ \\
\hline 52694 & 29 & $7380_{443}^{506}$ & $0.12_{0.01}^{0.01}$ \\
\hline 52701 & 36 & $7180_{419}^{473}$ & $0.13_{0.01}^{0.01}$ \\
\hline 52704 & 39 & $7040_{405}^{448}$ & $0.12_{0.01}^{0.01}$ \\
\hline 52707 & 42 & $6700_{355}^{412}$ & $0.12_{0.00}^{0.01}$ \\
\hline 52712 & 47 & $7560_{574}^{681}$ & $0.15_{0.01}^{0.02}$ \\
\hline 52717 & 52 & $6860_{954}^{1378}$ & $0.14_{0.02}^{0.03}$ \\
\hline 52722 & 57 & $6280_{606}^{781}$ & $0.12_{0.01}^{0.01}$ \\
\hline 52727 & 62 & $5900_{275}^{306}$ & $0.12_{0.00}^{0.00}$ \\
\hline 52734 & 69 & $6220_{324}^{351}$ & $0.12_{0.00}^{0.00}$ \\
\hline 52739 & 74 & $5600_{256}^{262}$ & $0.11_{0.00}^{0.00}$ \\
\hline 52751 & 86 & $6180_{1707}^{4384}$ & $0.11_{0.01}^{0.04}$ \\
\hline 52766 & 101 & $4840_{605}^{824}$ & $0.10_{0.01}^{0.01}$ \\
\hline 52771 & 106 & $5640_{290}^{323}$ & $0.09_{0.00}^{0.00}$ \\
\hline 52778 & 113 & $5900_{289}^{315}$ & $0.07_{0.00}^{0.00}$ \\
\hline \multicolumn{4}{|c|}{ SN2004A } \\
\hline 53018 & 11 & $7960_{337}^{369}$ & $1.06_{0.04}^{0.04}$ \\
\hline 53032 & 25 & $6360_{192}^{197}$ & $0.90_{0.02}^{0.02}$ \\
\hline 53037 & 30 & $6022_{510}^{592}$ & $0.87_{0.03}^{0.03}$ \\
\hline 53045 & 38 & $5860_{438}^{529}$ & $0.88_{0.03}^{0.04}$ \\
\hline 53050 & 43 & $5860_{348}^{409}$ & $0.89_{0.03}^{0.03}$ \\
\hline 53052 & 45 & $5900_{297}^{329}$ & $0.89_{0.02}^{0.03}$ \\
\hline
\end{tabular}


Faran et al.

Table A1 - continued A List of the Temperatures and Bolometric Luminosities

\begin{tabular}{|c|c|c|c|}
\hline 53065 & 58 & $5740_{282}^{309}$ & $0.90_{0.02}^{0.02}$ \\
\hline 53066 & 59 & $5760_{276}^{320}$ & $0.91_{0.02}^{0.02}$ \\
\hline 53076 & 69 & $5700_{281}^{300}$ & $0.91_{0.02}^{0.02}$ \\
\hline 53081 & 74 & $5710_{277}^{309}$ & $0.90_{0.03}^{0.03}$ \\
\hline 53099 & 92 & $5620_{264}^{302}$ & $0.82_{0.02}^{0.02}$ \\
\hline \multicolumn{4}{|c|}{ SN2004du } \\
\hline 53231 & 3 & $14644_{1658}^{2903}$ & $4.53_{0.75}^{1.64}$ \\
\hline 53233 & 5 & $11420_{620}^{700}$ & $3.61_{0.30}^{0.36}$ \\
\hline 53235 & 7 & $10140_{475}^{539}$ & $3.35_{0.21}^{0.25}$ \\
\hline 53237 & 9 & $9500_{418}^{458}$ & $3.21_{0.17}^{0.20}$ \\
\hline 53239 & 11 & $9120_{381}^{419}$ & $3.06_{0.15}^{0.17}$ \\
\hline 53242 & 14 & $8900_{361}^{397}$ & $2.93_{0.13}^{0.15}$ \\
\hline 53244 & 16 & $8680_{347}^{370}$ & $2.70_{0.11}^{0.13}$ \\
\hline 53246 & 18 & $8500_{415}^{457}$ & $2.55_{0.12}^{0.14}$ \\
\hline 53250 & 22 & $8153_{499}^{707}$ & $2.31_{0.15}^{0.25}$ \\
\hline 53251 & 23 & $8160_{542}^{650}$ & $2.26_{0.16}^{0.22}$ \\
\hline 53253 & 25 & $8120_{549}^{625}$ & $2.21_{0.16}^{0.20}$ \\
\hline 53255 & 27 & $8040_{537}^{611}$ & $2.18_{0.15}^{0.20}$ \\
\hline 53257 & 29 & $7920_{505}^{610}$ & $2.13_{0.14}^{0.19}$ \\
\hline 53259 & 31 & $7780_{483}^{590}$ & $2.07_{0.13}^{0.17}$ \\
\hline 53263 & 35 & $7480_{460}^{517}$ & $1.94_{0.11}^{0.14}$ \\
\hline 53265 & 37 & $7320_{437}^{495}$ & $1.88_{0.10}^{0.12}$ \\
\hline 53267 & 39 & $7180_{407}^{489}$ & $1.82_{0.08}^{0.12}$ \\
\hline 53269 & 41 & $7120_{411}^{465}$ & $1.79_{0.08}^{0.11}$ \\
\hline 53271 & 43 & $7060_{400}^{462}$ & $1.75_{0.08}^{0.10}$ \\
\hline 53273 & 45 & $7160_{553}^{678}$ & $1.78_{0.11}^{0.17}$ \\
\hline 53276 & 48 & $6760_{406}^{449}$ & $1.65_{0.07}^{0.09}$ \\
\hline 53279 & 51 & $6420_{326}^{373}$ & $1.58_{0.05}^{0.06}$ \\
\hline 53282 & 54 & $6380_{323}^{366}$ & $1.57_{0.05}^{0.06}$ \\
\hline 53285 & 57 & $6360_{319}^{366}$ & $1.55_{0.05}^{0.06}$ \\
\hline 53288 & 60 & $6320_{328}^{344}$ & $1.53_{0.05}^{0.05}$ \\
\hline 53292 & 64 & $6180_{304}^{338}$ & $1.51_{0.04}^{0.05}$ \\
\hline 53301 & 73 & $5780_{325}^{362}$ & $1.47_{0.04}^{0.05}$ \\
\hline 53309 & 81 & $5980_{279}^{321}$ & $1.41_{0.04}^{0.04}$ \\
\hline 53329 & 101 & $5340_{290}^{316}$ & $1.26_{0.04}^{0.04}$ \\
\hline \multicolumn{4}{|c|}{ SN2004et } \\
\hline 53278 & 7 & $11580_{677}^{772}$ & $2.42_{0.22}^{0.27}$ \\
\hline 53279 & 8 & $11280_{648}^{715}$ & $2.39_{0.21}^{0.25}$ \\
\hline 53280 & 9 & $10900_{600}^{664}$ & $2.30_{0.18}^{0.22}$ \\
\hline 53281 & 10 & $10620_{548}^{648}$ & $2.22_{0.16}^{0.21}$ \\
\hline 53282 & 11 & $10380_{525}^{610}$ & $2.15_{0.15}^{0.19}$ \\
\hline 53283 & 12 & $10140_{512}^{562}$ & $2.09_{0.14}^{0.17}$ \\
\hline 53284 & 13 & $10020_{500}^{545}$ & $2.06_{0.14}^{0.16}$ \\
\hline
\end{tabular}


Table A1 - continued A List of the Temperatures and Bolometric Luminosities

\begin{tabular}{|c|c|c|c|}
\hline 53286 & 15 & $9750_{473}^{538}$ & $2.00_{0.12}^{0.15}$ \\
\hline 53287 & 16 & $9680_{462}^{507}$ & $1.98_{0.12}^{0.14}$ \\
\hline 53288 & 17 & $9600_{447}^{506}$ & $1.97_{0.11}^{0.14}$ \\
\hline 53289 & 18 & $9500_{432}^{500}$ & $1.96_{0.11}^{0.14}$ \\
\hline 53294 & 23 & $8300_{195}^{201}$ & $1.65_{0.06}^{0.06}$ \\
\hline 53296 & 25 & $8021_{235}^{411}$ & $1.57_{0.07}^{0.14}$ \\
\hline 53297 & 26 & $7989_{264}^{306}$ & $1.58_{0.08}^{0.10}$ \\
\hline 53298 & 27 & $7880_{235}^{248}$ & $1.55_{0.07}^{0.08}$ \\
\hline 53299 & 28 & $7760_{209}^{239}$ & $1.51_{0.07}^{0.08}$ \\
\hline 53301 & 30 & $7600_{213}^{214}$ & $1.46_{0.06}^{0.07}$ \\
\hline 53302 & 31 & $7340_{185}^{212}$ & $1.42_{0.06}^{0.07}$ \\
\hline 53306 & 35 & $7120_{162}^{169}$ & $1.35_{0.05}^{0.05}$ \\
\hline 53307 & 36 & $7040_{153}^{170}$ & $1.32_{0.05}^{0.05}$ \\
\hline 53309 & 38 & $6960_{145}^{172}$ & $1.30_{0.04}^{0.05}$ \\
\hline 53312 & 41 & $6860_{146}^{160}$ & $1.27_{0.04}^{0.05}$ \\
\hline 53315 & 44 & $6740_{152}^{141}$ & $1.23_{0.04}^{0.04}$ \\
\hline 53316 & 45 & $6700_{149}^{140}$ & $1.22_{0.04}^{0.04}$ \\
\hline 53317 & 46 & $6660_{144}^{141}$ & $1.20_{0.04}^{0.04}$ \\
\hline 53318 & 47 & $6640_{143}^{140}$ & $1.20_{0.04}^{0.04}$ \\
\hline 53319 & 48 & $6610_{144}^{153}$ & $1.19_{0.04}^{0.04}$ \\
\hline 53320 & 49 & $6590_{141}^{146}$ & $1.19_{0.04}^{0.04}$ \\
\hline 53324 & 53 & $6520_{153}^{244}$ & $1.18_{0.04}^{0.08}$ \\
\hline 53326 & 55 & $6520_{180}^{197}$ & $1.19_{0.06}^{0.06}$ \\
\hline 53327 & 56 & $6500_{178}^{196}$ & $1.18_{0.11}^{0.21}$ \\
\hline 53328 & 57 & $6480_{179}^{194}$ & $1.18_{0.06}^{0.06}$ \\
\hline 53329 & 58 & $6480_{177}^{197}$ & $1.18_{0.06}^{0.06}$ \\
\hline 53330 & 59 & $6480_{186}^{187}$ & $1.18_{0.06}^{0.06}$ \\
\hline 53331 & 60 & $6450_{187}^{192}$ & $1.17_{0.06}^{0.06}$ \\
\hline 53332 & 61 & $6420_{177}^{191}$ & $1.16_{0.06}^{0.06}$ \\
\hline 53333 & 62 & $6400_{173}^{192}$ & $1.16_{0.06}^{0.06}$ \\
\hline 53335 & 64 & $6360_{181}^{186}$ & $1.14_{0.05}^{0.06}$ \\
\hline 53350 & 79 & $6480_{242}^{256}$ & $1.13_{0.15}^{0.33}$ \\
\hline 53353 & 82 & $6266_{260}^{395}$ & $1.08_{0.07}^{0.08}$ \\
\hline 53354 & 83 & $6340_{211}^{219}$ & $1.08_{0.05}^{0.06}$ \\
\hline 53355 & 84 & $6320_{196}^{234}$ & $1.08_{0.05}^{0.06}$ \\
\hline 53375 & 104 & $6020_{153}^{162}$ & $0.84_{0.05}^{0.06}$ \\
\hline 53376 & 105 & $6080_{161}^{160}$ & $0.83_{0.05}^{0.08}$ \\
\hline 53378 & 107 & $6020_{153}^{162}$ & $0.78_{0.04}^{0.06}$ \\
\hline 53381 & 110 & $5920_{156}^{147}$ & $0.71_{0.14}^{0.26}$ \\
\hline 53406 & 135 & $5880_{194}^{205}$ & $0.20_{0.04}^{0.07}$ \\
\hline 53412 & 141 & $5940_{203}^{214}$ & $0.17_{0.04}^{0.04}$ \\
\hline \multicolumn{4}{|c|}{ SN2005cs } \\
\hline 53552 & 3 & $16327_{684}^{745}$ & $0.89_{0.07}^{0.08}$ \\
\hline 53553 & 4 & $15060_{749}^{801}$ & $0.78_{0.07}^{0.08}$ \\
\hline
\end{tabular}


Faran et al.

Table A1 - continued A List of the Temperatures and Bolometric Luminosities

\begin{tabular}{|c|c|c|c|}
\hline 53554 & 5 & $13509_{623}^{693}$ & $0.63_{0.05}^{0.06}$ \\
\hline 53555 & 6 & $12356_{692}^{846}$ & $0.54_{0.04}^{0.06}$ \\
\hline 53557 & 8 & $10418_{668}^{788}$ & $0.42_{0.03}^{0.03}$ \\
\hline 53558 & 9 & $9690_{298}^{317}$ & $0.38_{0.02}^{0.02}$ \\
\hline 53559 & 10 & $9540_{271}^{295}$ & $0.38_{0.02}^{0.02}$ \\
\hline 53560 & 11 & $9269_{364}^{374}$ & $0.36_{0.02}^{0.02}$ \\
\hline 53562 & 13 & $8376_{333}^{468}$ & $0.31_{0.02}^{0.03}$ \\
\hline 53563 & 14 & $8088_{230}^{246}$ & $0.30_{0.02}^{0.02}$ \\
\hline 53564 & 15 & $7967_{220}^{247}$ & $0.29_{0.01}^{0.02}$ \\
\hline 53565 & 16 & $7708_{219}^{242}$ & $0.27_{0.01}^{0.01}$ \\
\hline 53566 & 17 & $7560_{217}^{242}$ & $0.27_{0.01}^{0.01}$ \\
\hline 53568 & 19 & $7360_{191}^{195}$ & $0.26_{0.01}^{0.01}$ \\
\hline 53569 & 20 & $7260_{175}^{200}$ & $0.25_{0.01}^{0.01}$ \\
\hline 53571 & 22 & $6960_{177}^{192}$ & $0.24_{0.01}^{0.01}$ \\
\hline 53572 & 23 & $6920_{178}^{186}$ & $0.24_{0.01}^{0.01}$ \\
\hline 53573 & 24 & $6967_{176}^{175}$ & $0.24_{0.01}^{0.01}$ \\
\hline 53574 & 25 & $6880_{147}^{159}$ & $0.24_{0.01}^{0.01}$ \\
\hline 53575 & 26 & $6700_{142}^{150}$ & $0.24_{0.01}^{0.01}$ \\
\hline 53577 & 28 & $6720_{150}^{178}$ & $0.24_{0.01}^{0.01}$ \\
\hline 53579 & 30 & $6670_{162}^{167}$ & $0.24_{0.01}^{0.01}$ \\
\hline 53580 & 31 & $6760_{200}^{204}$ & $0.24_{0.01}^{0.01}$ \\
\hline 53583 & 34 & $6660_{186}^{184}$ & $0.24_{0.01}^{0.01}$ \\
\hline 53584 & 35 & $6604_{180}^{227}$ & $0.25_{0.01}^{0.01}$ \\
\hline 53585 & 36 & $6505_{180}^{202}$ & $0.24_{0.01}^{0.01}$ \\
\hline 53586 & 37 & $6700_{228}^{251}$ & $0.26_{0.01}^{0.01}$ \\
\hline 53588 & 39 & $6560_{225}^{232}$ & $0.26_{0.01}^{0.01}$ \\
\hline 53589 & 40 & $6500_{220}^{227}$ & $0.26_{0.01}^{0.01}$ \\
\hline 53591 & 42 & $6360_{176}^{196}$ & $0.26_{0.01}^{0.01}$ \\
\hline 53593 & 44 & $6360_{177}^{187}$ & $0.26_{0.01}^{0.02}$ \\
\hline 53595 & 46 & $6360_{188}^{210}$ & $0.27_{0.02}^{0.02}$ \\
\hline 53599 & 50 & $6360_{183}^{180}$ & $0.27_{0.01}^{0.01}$ \\
\hline 53600 & 51 & $6340_{175}^{187}$ & $0.27_{0.01}^{0.01}$ \\
\hline 53605 & 56 & $6250_{182}^{196}$ & $0.28_{0.01}^{0.02}$ \\
\hline 53606 & 57 & $6360_{180}^{184}$ & $0.29_{0.01}^{0.01}$ \\
\hline 53610 & 61 & $6203_{247}^{230}$ & $0.28_{0.02}^{0.02}$ \\
\hline 53612 & 63 & $6260_{175}^{168}$ & $0.29_{0.01}^{0.01}$ \\
\hline 53613 & 64 & $6320_{181}^{184}$ & $0.30_{0.01}^{0.01}$ \\
\hline 53615 & 66 & $6310_{182}^{182}$ & $0.30_{0.01}^{0.01}$ \\
\hline 53617 & 68 & $6220_{171}^{167}$ & $0.29_{0.01}^{0.01}$ \\
\hline 53619 & 70 & $6200_{156}^{181}$ & $0.29_{0.01}^{0.01}$ \\
\hline 53624 & 75 & $6100_{196}^{199}$ & $0.29_{0.01}^{0.01}$ \\
\hline 53626 & 77 & $6240_{157}^{185}$ & $0.30_{0.01}^{0.01}$ \\
\hline 53628 & 79 & $6220_{159}^{182}$ & $0.30_{0.01}^{0.01}$ \\
\hline 53629 & 80 & $6400_{687}^{906}$ & $0.33_{0.01}^{0.01}$ \\
\hline 53640 & 91 & $5520_{568}^{568}$ & $0.24_{0.01}^{0.01}$ \\
\hline
\end{tabular}


Table A1 - continued A List of the Temperatures and Bolometric Luminosities

\begin{tabular}{|c|c|c|c|}
\hline 53642 & 93 & $5520_{460}^{583}$ & $0.24_{0.01}^{0.02}$ \\
\hline 53781 & 232 & $4080_{283}^{338}$ & $0.01_{0.00}^{0.01}$ \\
\hline \multicolumn{4}{|c|}{ SN2006bp } \\
\hline 53836 & 2 & $10662_{632}^{480}$ & $0.90_{0.06}^{0.06}$ \\
\hline 53837 & 3 & $9779_{208}^{267}$ & $0.91_{0.04}^{0.04}$ \\
\hline 53838 & 4 & $9380_{152}^{163}$ & $0.95_{0.02}^{0.02}$ \\
\hline 53839 & 5 & $8713_{215}^{171}$ & $0.97_{0.02}^{0.02}$ \\
\hline 53840 & 6 & $8292_{173}^{225}$ & $0.97_{0.02}^{0.02}$ \\
\hline 53841 & 7 & $8085_{155}^{158}$ & $0.96_{0.02}^{0.02}$ \\
\hline 53842 & 8 & $7796_{169}^{175}$ & $0.95_{0.02}^{0.02}$ \\
\hline 53843 & 9 & $7447_{135}^{150}$ & $0.93_{0.02}^{0.02}$ \\
\hline 53844 & 10 & $7176_{269}^{156}$ & $0.92_{0.02}^{0.02}$ \\
\hline 53846 & 12 & $6724_{130}^{143}$ & $0.89_{0.02}^{0.02}$ \\
\hline 53847 & 13 & $6640_{126}^{124}$ & $0.88_{0.02}^{0.02}$ \\
\hline 53848 & 14 & $6513_{170}^{169}$ & $0.87_{0.02}^{0.02}$ \\
\hline 53849 & 15 & $6360_{215}^{231}$ & $0.85_{0.02}^{0.02}$ \\
\hline 53850 & 16 & $6290_{190}^{203}$ & $0.84_{0.02}^{0.02}$ \\
\hline 53852 & 18 & $6050_{186}^{181}$ & $0.84_{0.02}^{0.02}$ \\
\hline 53854 & 20 & $5782_{198}^{467}$ & $0.83_{0.02}^{0.02}$ \\
\hline 53857 & 23 & $5880_{313}^{365}$ & $0.84_{0.02}^{0.02}$ \\
\hline 53858 & 24 & $5840_{314}^{345}$ & $0.84_{0.02}^{0.02}$ \\
\hline 53859 & 25 & $5740_{298}^{338}$ & $0.84_{0.02}^{0.02}$ \\
\hline 53860 & 26 & $5680_{291}^{335}$ & $0.83_{0.02}^{0.02}$ \\
\hline 53861 & 27 & $5560_{285}^{314}$ & $0.83_{0.02}^{0.03}$ \\
\hline 53862 & 28 & $5580_{292}^{312}$ & $0.83_{0.02}^{0.03}$ \\
\hline 53866 & 32 & $5420_{273}^{297}$ & $0.81_{0.02}^{0.03}$ \\
\hline 53867 & 33 & $5340_{253}^{294}$ & $0.81_{0.02}^{0.03}$ \\
\hline 53870 & 36 & $5300_{264}^{271}$ & $0.78_{0.02}^{0.03}$ \\
\hline 53886 & 52 & $4960_{229}^{248}$ & $0.74_{0.03}^{0.04}$ \\
\hline \multicolumn{4}{|c|}{ SN2007od } \\
\hline 54410 & 11 & $8720_{504}^{582}$ & $6.60_{0.31}^{0.37}$ \\
\hline 54411 & 12 & $8640_{520}^{577}$ & $6.55_{0.32}^{0.36}$ \\
\hline 54412 & 13 & $8420_{330}^{355}$ & $6.55_{0.29}^{0.32}$ \\
\hline 54413 & 14 & $8500_{288}^{330}$ & $6.34_{0.24}^{0.30}$ \\
\hline 54414 & 15 & $8386_{180}^{195}$ & $6.17_{0.25}^{0.37}$ \\
\hline 54416 & 17 & $8140_{157}^{153}$ & $5.72_{0.21}^{0.22}$ \\
\hline 54417 & 18 & $8000_{180}^{188}$ & $5.49_{0.24}^{0.24}$ \\
\hline 54418 & 19 & $7904_{233}^{203}$ & $5.34_{0.29}^{0.26}$ \\
\hline 54422 & 23 & $7846_{223}^{299}$ & $5.18_{0.28}^{0.45}$ \\
\hline 54426 & 27 & $7680_{172}^{201}$ & $4.96_{0.19}^{0.23}$ \\
\hline 54428 & 29 & $7600_{184}^{186}$ & $4.79_{0.20}^{0.21}$ \\
\hline 54429 & 30 & $7580_{176}^{186}$ & $4.75_{0.19}^{0.21}$ \\
\hline 54433 & 34 & $7500_{167}^{187}$ & $4.56_{0.18}^{0.19}$ \\
\hline
\end{tabular}


Faran et al.

Table A1 - continued A List of the Temperatures and Bolometric Luminosities

\begin{tabular}{|c|c|c|c|}
\hline 54436 & 37 & $7420_{192}^{201}$ & $4.46_{0.20}^{0.22}$ \\
\hline 54439 & 40 & $7150_{232}^{245}$ & $4.12_{0.23}^{0.27}$ \\
\hline 54441 & 42 & $7060_{168}^{161}$ & $3.99_{0.16}^{0.15}$ \\
\hline 54442 & 43 & $7000_{154}^{145}$ & $3.91_{0.14}^{0.13}$ \\
\hline 54446 & 47 & $6860_{148}^{137}$ & $3.70_{0.12}^{0.12}$ \\
\hline 54449 & 50 & $6690_{146}^{149}$ & $3.46_{0.13}^{0.13}$ \\
\hline 54450 & 51 & $6800_{152}^{146}$ & $3.54_{0.13}^{0.13}$ \\
\hline 54451 & 52 & $6800_{155}^{149}$ & $3.54_{0.13}^{0.13}$ \\
\hline 54456 & 57 & $6660_{167}^{184}$ & $3.40_{0.15}^{0.17}$ \\
\hline 54460 & 61 & $6446_{190}^{319}$ & $3.05_{0.16}^{0.34}$ \\
\hline 54462 & 63 & $6440_{138}^{128}$ & $2.96_{0.10}^{0.10}$ \\
\hline 54464 & 65 & $6600_{144}^{160}$ & $3.06_{0.11}^{0.12}$ \\
\hline 54473 & 74 & $6720_{131}^{149}$ & $2.48_{0.08}^{0.09}$ \\
\hline \multicolumn{4}{|c|}{ SN2008in } \\
\hline 54830 & 8 & $10600_{168}^{193}$ & $0.70_{0.02}^{0.02}$ \\
\hline 54831 & 9 & $9965_{560}^{741}$ & $0.64_{0.05}^{0.07}$ \\
\hline 54833 & 11 & $9383_{456}^{519}$ & $0.57_{0.03}^{0.04}$ \\
\hline 54835 & 13 & $9123_{399}^{401}$ & $0.54_{0.03}^{0.03}$ \\
\hline 54839 & 17 & $7575_{285}^{371}$ & $0.44_{0.02}^{0.03}$ \\
\hline 54841 & 19 & $7519_{282}^{312}$ & $0.45_{0.02}^{0.02}$ \\
\hline 54843 & 21 & $7540_{307}^{345}$ & $0.44_{0.02}^{0.02}$ \\
\hline 54846 & 24 & $7300_{303}^{338}$ & $0.42_{0.02}^{0.02}$ \\
\hline 54851 & 29 & $6919_{275}^{354}$ & $0.40_{0.01}^{0.02}$ \\
\hline 54855 & 33 & $6600_{256}^{284}$ & $0.38_{0.01}^{0.02}$ \\
\hline 54856 & 34 & $6420_{203}^{212}$ & $0.37_{0.01}^{0.01}$ \\
\hline 54858 & 36 & $6340_{183}^{192}$ & $0.36_{0.01}^{0.01}$ \\
\hline 54860 & 38 & $6320_{185}^{183}$ & $0.36_{0.01}^{0.01}$ \\
\hline 54861 & 39 & $6240_{149}^{175}$ & $0.36_{0.01}^{0.01}$ \\
\hline 54862 & 40 & $6240_{170}^{198}$ & $0.36_{0.01}^{0.01}$ \\
\hline 54864 & 42 & $6220_{176}^{185}$ & $0.36_{0.01}^{0.01}$ \\
\hline 54868 & 46 & $6140_{181}^{205}$ & $0.36_{0.01}^{0.01}$ \\
\hline 54874 & 52 & $6220_{261}^{266}$ & $0.35_{0.01}^{0.01}$ \\
\hline 54878 & 56 & $6100_{206}^{243}$ & $0.34_{0.01}^{0.01}$ \\
\hline 54879 & 57 & $6084_{241}^{396}$ & $0.34_{0.01}^{0.01}$ \\
\hline 54880 & 58 & $6240_{212}^{227}$ & $0.34_{0.01}^{0.01}$ \\
\hline 54883 & 61 & $6140_{197}^{229}$ & $0.33_{0.01}^{0.01}$ \\
\hline 54884 & 62 & $6180_{195}^{213}$ & $0.33_{0.01}^{0.01}$ \\
\hline 54885 & 63 & $6170_{212}^{226}$ & $0.33_{0.01}^{0.01}$ \\
\hline 54886 & 64 & $6220_{267}^{299}$ & $0.33_{0.02}^{0.02}$ \\
\hline 54888 & 66 & $6180_{258}^{302}$ & $0.32_{0.01}^{0.02}$ \\
\hline 54889 & 67 & $6060_{182}^{197}$ & $0.31_{0.01}^{0.01}$ \\
\hline 54890 & 68 & $6060_{190}^{208}$ & $0.31_{0.01}^{0.01}$ \\
\hline 54893 & 71 & $6060_{206}^{209}$ & $0.30_{0.01}^{0.01}$ \\
\hline 54895 & 73 & $6021_{238}^{232}$ & $0.30_{0.01}^{0.01}$ \\
\hline
\end{tabular}


Table A1 - continued A List of the Temperatures and Bolometric Luminosities

\begin{tabular}{|c|c|c|c|}
\hline 54900 & 78 & $5940_{271}^{286}$ & $0.29_{0.01}^{0.01}$ \\
\hline 54901 & 79 & $6460_{542}^{679}$ & $0.31_{0.02}^{0.03}$ \\
\hline 54906 & 84 & $5880_{246}^{257}$ & $0.28_{0.01}^{0.01}$ \\
\hline 54912 & 90 & $6000_{288}^{335}$ & $0.26_{0.01}^{0.01}$ \\
\hline 54915 & 93 & $5840_{187}^{193}$ & $0.24_{0.01}^{0.01}$ \\
\hline 54917 & 95 & $5860_{167}^{193}$ & $0.23_{0.01}^{0.01}$ \\
\hline 54925 & 103 & $5960_{225}^{248}$ & $0.20_{0.01}^{0.01}$ \\
\hline 54927 & 105 & $5649_{213}^{250}$ & $0.17_{0.00}^{0.01}$ \\
\hline 54932 & 110 & $5240_{204}^{207}$ & $0.12_{0.00}^{0.00}$ \\
\hline 54937 & 115 & $4279_{281}^{170}$ & $0.08_{0.00}^{0.00}$ \\
\hline \multicolumn{4}{|c|}{ SN2009N } \\
\hline 54858 & 13 & $10120_{711}^{835}$ & $0.57_{0.03}^{0.04}$ \\
\hline 54859 & 14 & $8154_{259}^{256}$ & $0.53_{0.02}^{0.02}$ \\
\hline 54860 & 15 & $8050_{220}^{236}$ & $0.53_{0.01}^{0.01}$ \\
\hline 54861 & 16 & $7895_{217}^{232}$ & $0.52_{0.01}^{0.01}$ \\
\hline 54863 & 18 & $7220_{143}^{154}$ & $0.50_{0.01}^{0.01}$ \\
\hline 54864 & 19 & $6940_{142}^{148}$ & $0.49_{0.01}^{0.01}$ \\
\hline 54865 & 20 & $6600_{123}^{138}$ & $0.49_{0.01}^{0.01}$ \\
\hline 54867 & 22 & $6413_{97}^{120}$ & $0.47_{0.02}^{0.02}$ \\
\hline 54869 & 24 & $6320_{134}^{135}$ & $0.47_{0.01}^{0.01}$ \\
\hline 54871 & 26 & $6160_{121}^{132}$ & $0.46_{0.01}^{0.01}$ \\
\hline 54872 & 27 & $6100_{130}^{118}$ & $0.46_{0.01}^{0.01}$ \\
\hline 54875 & 30 & $5900_{113}^{123}$ & $0.46_{0.01}^{0.01}$ \\
\hline 54878 & 33 & $5800_{124}^{148}$ & $0.47_{0.02}^{0.02}$ \\
\hline 54880 & 35 & $5750_{130}^{140}$ & $0.48_{0.02}^{0.02}$ \\
\hline 54887 & 42 & $5500_{118}^{122}$ & $0.49_{0.01}^{0.02}$ \\
\hline 54888 & 43 & $5480_{113}^{126}$ & $0.50_{0.01}^{0.02}$ \\
\hline 54889 & 44 & $5480_{116}^{125}$ & $0.50_{0.01}^{0.01}$ \\
\hline 54890 & 45 & $5480_{124}^{114}$ & $0.50_{0.01}^{0.01}$ \\
\hline 54891 & 46 & $5460_{117}^{120}$ & $0.51_{0.01}^{0.01}$ \\
\hline 54896 & 51 & $5340_{104}^{123}$ & $0.52_{0.01}^{0.01}$ \\
\hline 54897 & 52 & $5340_{103}^{123}$ & $0.52_{0.01}^{0.01}$ \\
\hline 54902 & 57 & $5320_{109}^{115}$ & $0.53_{0.01}^{0.01}$ \\
\hline 54904 & 59 & $5320_{101}^{123}$ & $0.53_{0.01}^{0.01}$ \\
\hline 54906 & 61 & $5340_{112}^{114}$ & $0.54_{0.01}^{0.01}$ \\
\hline 54907 & 62 & $5360_{115}^{113}$ & $0.53_{0.01}^{0.01}$ \\
\hline 54908 & 63 & $5360_{114}^{113}$ & $0.53_{0.01}^{0.01}$ \\
\hline 54909 & 64 & $5360_{116}^{112}$ & $0.53_{0.01}^{0.01}$ \\
\hline 54910 & 65 & $5340_{116}^{109}$ & $0.54_{0.01}^{0.01}$ \\
\hline 54911 & 66 & $5320_{101}^{124}$ & $0.54_{0.01}^{0.01}$ \\
\hline 54913 & 68 & $5320_{114}^{112}$ & $0.54_{0.01}^{0.01}$ \\
\hline 54915 & 70 & $5300_{104}^{119}$ & $0.53_{0.01}^{0.01}$ \\
\hline 54916 & 71 & $5300_{110}^{112}$ & $0.53_{0.01}^{0.01}$ \\
\hline 54917 & 72 & $5280_{100}^{123}$ & $0.53_{0.01}^{0.01}$ \\
\hline
\end{tabular}


Faran et al.

Table A1 - continued A List of the Temperatures and Bolometric Luminosities

\begin{tabular}{llll}
54918 & 73 & $5280_{101}^{120}$ & $0.53_{0.01}^{0.01}$ \\
54919 & 74 & $5280_{105}^{117}$ & $0.53_{0.01}^{0.01}$ \\
54920 & 75 & $5280_{105}^{115}$ & $0.53_{0.01}^{0.01}$ \\
54946 & 101 & $5300_{109}^{113}$ & $0.39_{0.01}^{0.01}$ \\
54947 & 102 & $5060_{122}^{109}$ & $0.39_{0.01}^{0.01}$ \\
\hline
\end{tabular}

\section{SN2009bw}

\begin{tabular}{|c|c|c|c|}
\hline 54923 & 6 & $15826_{757}^{988}$ & $2.29_{0.09}^{0.64}$ \\
\hline 54924 & 7 & $14043_{356}^{365}$ & $2.16_{0.08}^{0.08}$ \\
\hline 54925 & 8 & $11720_{238}^{233}$ & $1.73_{0.05}^{0.06}$ \\
\hline 54926 & 9 & $11022_{534}^{293}$ & $1.61_{0.10}^{0.08}$ \\
\hline 54927 & 10 & $10360_{337}^{350}$ & $1.47_{0.06}^{0.06}$ \\
\hline 54930 & 13 & $9240_{277}^{296}$ & $1.25_{0.04}^{0.05}$ \\
\hline 54931 & 14 & $9040_{280}^{281}$ & $1.20_{0.04}^{0.04}$ \\
\hline 54932 & 15 & $8891_{328}^{324}$ & $1.16_{0.05}^{0.05}$ \\
\hline 54935 & 18 & $8324_{602}^{333}$ & $1.00_{0.07}^{0.04}$ \\
\hline 54937 & 20 & $7638_{177}^{214}$ & $0.86_{0.02}^{0.02}$ \\
\hline 54942 & 25 & $7090_{443}^{502}$ & $0.79_{0.04}^{0.05}$ \\
\hline 54945 & 28 & $6820_{400}^{462}$ & $0.75_{0.03}^{0.04}$ \\
\hline 54946 & 29 & $6780_{386}^{465}$ & $0.74_{0.03}^{0.04}$ \\
\hline 54949 & 32 & $6500_{367}^{404}$ & $0.68_{0.03}^{0.03}$ \\
\hline 54951 & 34 & $6840_{1130}^{1744}$ & $0.67_{0.08}^{0.19}$ \\
\hline 54952 & 35 & $6560_{362}^{430}$ & $0.64_{0.02}^{0.03}$ \\
\hline 54953 & 36 & $6400_{352}^{395}$ & $0.62_{0.02}^{0.03}$ \\
\hline 54956 & 39 & $5950_{310}^{340}$ & $0.59_{0.02}^{0.02}$ \\
\hline 54957 & 40 & $5760_{425}^{497}$ & $0.59_{0.02}^{0.02}$ \\
\hline 54961 & 44 & $5680_{354}^{424}$ & $0.56_{0.02}^{0.02}$ \\
\hline 54967 & 50 & $5480_{324}^{383}$ & $0.55_{0.02}^{0.02}$ \\
\hline 54971 & 54 & $5420_{246}^{288}$ & $0.52_{0.02}^{0.02}$ \\
\hline 54974 & 57 & $5420_{244}^{279}$ & $0.52_{0.01}^{0.01}$ \\
\hline 54978 & 61 & $5400_{516}^{661}$ & $0.51_{0.04}^{0.03}$ \\
\hline 54979 & 62 & $5460_{692}^{935}$ & $0.52_{0.03}^{0.04}$ \\
\hline 54983 & 66 & $5600_{270}^{289}$ & $0.51_{0.01}^{0.01}$ \\
\hline 54984 & 67 & $5540_{1406}^{2929}$ & $0.51_{0.05}^{0.23}$ \\
\hline 55010 & 93 & $5450_{295}^{1079}$ & $0.45_{0.01}^{0.05}$ \\
\hline 55033 & 116 & $5720_{581}^{731}$ & $0.36_{0.02}^{0.03}$ \\
\hline 55037 & 120 & $5800_{590}^{765}$ & $0.34_{0.02}^{0.03}$ \\
\hline 55040 & 123 & $5900_{792}^{1080}$ & $0.33_{0.02}^{0.04}$ \\
\hline 55044 & 127 & $6236_{1297}^{1920}$ & $0.31_{0.03}^{0.10}$ \\
\hline 55050 & 133 & $8500_{2183}^{4925}$ & $0.26_{0.07}^{0.32}$ \\
\hline
\end{tabular}

SN2009ib

\begin{tabular}{llll}
\hline 55054 & 13 & $9120_{200}^{188}$ & $0.76_{0.02}^{0.02}$ \\
55056 & 15 & $8192_{351}^{1031}$ & $0.67_{0.02}^{0.09}$ \\
55057 & 16 & $8104_{394}^{1041}$ & $0.66_{0.04}^{0.14}$
\end{tabular}


Table A1 - continued A List of the Temperatures and Bolometric Luminosities

\begin{tabular}{|c|c|c|c|}
\hline 55063 & 22 & $7860_{519}^{626}$ & $0.65_{0.07}^{0.10}$ \\
\hline 55064 & 23 & $7760_{496}^{590}$ & $0.64_{0.05}^{0.07}$ \\
\hline 55066 & 25 & $7330_{444}^{543}$ & $0.60_{0.04}^{0.07}$ \\
\hline 55067 & 26 & $7340_{453}^{512}$ & $0.60_{0.04}^{0.05}$ \\
\hline 55068 & 27 & $7109_{409}^{509}$ & $0.57_{0.04}^{0.05}$ \\
\hline 55069 & 28 & $7150_{410}^{479}$ & $0.59_{0.04}^{0.04}$ \\
\hline 55070 & 29 & $6920_{379}^{420}$ & $0.55_{0.03}^{0.04}$ \\
\hline 55071 & 30 & $6840_{365}^{415}$ & $0.55_{0.03}^{0.04}$ \\
\hline 55072 & 31 & $6818_{414}^{481}$ & $0.55_{0.04}^{0.04}$ \\
\hline 55073 & 32 & $6640_{308}^{357}$ & $0.54_{0.03}^{0.04}$ \\
\hline 55074 & 33 & $6660_{335}^{377}$ & $0.53_{0.02}^{0.03}$ \\
\hline 55075 & 34 & $6640_{330}^{381}$ & $0.54_{0.03}^{0.03}$ \\
\hline 55077 & 36 & $6380_{303}^{346}$ & $0.51_{0.02}^{0.03}$ \\
\hline 55079 & 38 & $6340_{310}^{332}$ & $0.51_{0.02}^{0.03}$ \\
\hline 55082 & 41 & $6260_{303}^{319}$ & $0.51_{0.02}^{0.02}$ \\
\hline 55083 & 42 & $6120_{284}^{312}$ & $0.50_{0.02}^{0.02}$ \\
\hline 55084 & 43 & $6139_{301}^{341}$ & $0.50_{0.02}^{0.02}$ \\
\hline 55085 & 44 & $6130_{278}^{322}$ & $0.50_{0.02}^{0.03}$ \\
\hline 55086 & 45 & $6140_{290}^{304}$ & $0.50_{0.02}^{0.02}$ \\
\hline 55087 & 46 & $6140_{284}^{321}$ & $0.51_{0.02}^{0.02}$ \\
\hline 55088 & 47 & $6100_{273}^{302}$ & $0.50_{0.02}^{0.02}$ \\
\hline 55089 & 48 & $5940_{221}^{239}$ & $0.49_{0.02}^{0.02}$ \\
\hline 55090 & 49 & $5950_{274}^{290}$ & $0.49_{0.02}^{0.02}$ \\
\hline 55091 & 50 & $5900_{265}^{290}$ & $0.49_{0.02}^{0.02}$ \\
\hline 55092 & 51 & $5870_{270}^{301}$ & $0.49_{0.02}^{0.02}$ \\
\hline 55093 & 52 & $5880_{262}^{289}$ & $0.49_{0.02}^{0.02}$ \\
\hline 55094 & 53 & $5880_{251}^{282}$ & $0.49_{0.02}^{0.02}$ \\
\hline 55095 & 54 & $5745_{318}^{514}$ & $0.48_{0.02}^{0.02}$ \\
\hline 55096 & 55 & $5960_{273}^{318}$ & $0.49_{0.01}^{0.02}$ \\
\hline 55097 & 56 & $5940_{278}^{307}$ & $0.49_{0.01}^{0.02}$ \\
\hline 55099 & 58 & $5840_{265}^{300}$ & $0.48_{0.02}^{0.02}$ \\
\hline 55100 & 59 & $5870_{294}^{322}$ & $0.48_{0.01}^{0.02}$ \\
\hline 55104 & 63 & $5840_{286}^{305}$ & $0.49_{0.01}^{0.02}$ \\
\hline 55105 & 64 & $5820_{272}^{316}$ & $0.49_{0.01}^{0.02}$ \\
\hline 55109 & 68 & $5760_{271}^{304}$ & $0.48_{0.01}^{0.02}$ \\
\hline 55111 & 70 & $5720_{259}^{308}$ & $0.48_{0.01}^{0.02}$ \\
\hline 55116 & 75 & $5360_{191}^{203}$ & $0.47_{0.01}^{0.02}$ \\
\hline 55117 & 76 & $5520_{237}^{251}$ & $0.48_{0.01}^{0.02}$ \\
\hline 55121 & 80 & $5540_{234}^{250}$ & $0.48_{0.01}^{0.02}$ \\
\hline 55122 & 81 & $5520_{230}^{234}$ & $0.48_{0.01}^{0.01}$ \\
\hline 55124 & 83 & $5500_{218}^{244}$ & $0.48_{0.01}^{0.01}$ \\
\hline 55128 & 87 & $5260_{154}^{146}$ & $0.48_{0.01}^{0.01}$ \\
\hline 55131 & 90 & $5500_{227}^{232}$ & $0.48_{0.01}^{0.01}$ \\
\hline 55132 & 91 & $5480_{214}^{244}$ & $0.48_{0.01}^{0.01}$ \\
\hline 55134 & 93 & $5480_{216}^{241}$ & $0.48_{0.01}^{0.01}$ \\
\hline
\end{tabular}


34 Faran et al.

Table A1 - continued A List of the Temperatures and Bolometric Luminosities

\begin{tabular}{|c|c|c|c|}
\hline 55137 & 96 & $5480_{219}^{237}$ & $0.48_{0.01}^{0.01}$ \\
\hline 55138 & 97 & $5460_{214}^{239}$ & $0.48_{0.01}^{0.01}$ \\
\hline 55141 & 100 & $5520_{230}^{259}$ & $0.48_{0.01}^{0.01}$ \\
\hline 55146 & 105 & $5460_{209}^{242}$ & $0.47_{0.01}^{0.01}$ \\
\hline 55147 & 106 & $5460_{213}^{237}$ & $0.47_{0.01}^{0.01}$ \\
\hline \multicolumn{4}{|c|}{ SN2012A } \\
\hline 55937 & 8 & $15960_{594}^{636}$ & $1.62_{0.11}^{0.12}$ \\
\hline 55938 & 9 & $13700_{641}^{1166}$ & $1.43_{0.14}^{0.21}$ \\
\hline 55939 & 10 & $12039_{503}^{567}$ & $1.17_{0.07}^{0.08}$ \\
\hline 55940 & 11 & $11043_{961}^{464}$ & $1.06_{0.15}^{0.09}$ \\
\hline 55941 & 12 & $10837_{600}^{710}$ & $1.04_{0.11}^{0.16}$ \\
\hline 55942 & 13 & $10665_{432}^{482}$ & $1.04_{0.06}^{0.08}$ \\
\hline 55943 & 14 & $10459_{441}^{535}$ & $1.02_{0.06}^{0.08}$ \\
\hline 55945 & 16 & $10106_{428}^{473}$ & $0.98_{0.06}^{0.07}$ \\
\hline 55948 & 19 & $9034_{653}^{709}$ & $0.82_{0.07}^{0.10}$ \\
\hline 55949 & 20 & $9020_{572}^{671}$ & $0.82_{0.07}^{0.09}$ \\
\hline 55951 & 22 & $8680_{661}^{787}$ & $0.79_{0.07}^{0.10}$ \\
\hline 55952 & 23 & $8577_{633}^{803}$ & $0.78_{0.07}^{0.11}$ \\
\hline 55953 & 24 & $8261_{622}^{1105}$ & $0.73_{0.07}^{0.17}$ \\
\hline 55954 & 25 & $7960_{416}^{490}$ & $0.70_{0.04}^{0.05}$ \\
\hline 55955 & 26 & $7800_{416}^{492}$ & $0.68_{0.04}^{0.05}$ \\
\hline 55957 & 28 & $7620_{384}^{403}$ & $0.64_{0.03}^{0.04}$ \\
\hline 55958 & 29 & $7519_{398}^{484}$ & $0.63_{0.03}^{0.05}$ \\
\hline 55962 & 33 & $7540_{408}^{453}$ & $0.63_{0.04}^{0.05}$ \\
\hline 55966 & 37 & $7099_{450}^{670}$ & $0.58_{0.04}^{0.07}$ \\
\hline 55967 & 38 & $7194_{519}^{552}$ & $0.59_{0.05}^{0.05}$ \\
\hline 55969 & 40 & $7179_{365}^{606}$ & $0.58_{0.03}^{0.05}$ \\
\hline 55972 & 43 & $7320_{453}^{538}$ & $0.58_{0.04}^{0.05}$ \\
\hline 55974 & 45 & $7320_{472}^{571}$ & $0.58_{0.04}^{0.05}$ \\
\hline 55975 & 46 & $7235_{442}^{592}$ & $0.58_{0.04}^{0.05}$ \\
\hline 55976 & 47 & $7220_{436}^{509}$ & $0.57_{0.04}^{0.05}$ \\
\hline 55977 & 48 & $7160_{422}^{504}$ & $0.57_{0.03}^{0.04}$ \\
\hline 55979 & 50 & $6959_{404}^{488}$ & $0.54_{0.03}^{0.04}$ \\
\hline 55981 & 52 & $6940_{408}^{503}$ & $0.53_{0.03}^{0.04}$ \\
\hline 55982 & 53 & $6940_{437}^{509}$ & $0.52_{0.03}^{0.04}$ \\
\hline 55983 & 54 & $6840_{404}^{483}$ & $0.52_{0.03}^{0.04}$ \\
\hline 55984 & 55 & $6840_{419}^{468}$ & $0.52_{0.03}^{0.04}$ \\
\hline 55985 & 56 & $6940_{413}^{491}$ & $0.52_{0.03}^{0.04}$ \\
\hline 55987 & 58 & $6680_{404}^{467}$ & $0.49_{0.03}^{0.04}$ \\
\hline 55988 & 59 & $6420_{363}^{414}$ & $0.47_{0.02}^{0.03}$ \\
\hline 55991 & 62 & $6520_{391}^{441}$ & $0.48_{0.03}^{0.03}$ \\
\hline 55992 & 63 & $6680_{354}^{401}$ & $0.49_{0.02}^{0.03}$ \\
\hline 55993 & 64 & $6760_{355}^{391}$ & $0.50_{0.03}^{0.03}$ \\
\hline 55994 & 65 & $6820_{365}^{392}$ & $0.50_{0.03}^{0.03}$ \\
\hline
\end{tabular}


Table A1 - continued A List of the Temperatures and Bolometric Luminosities

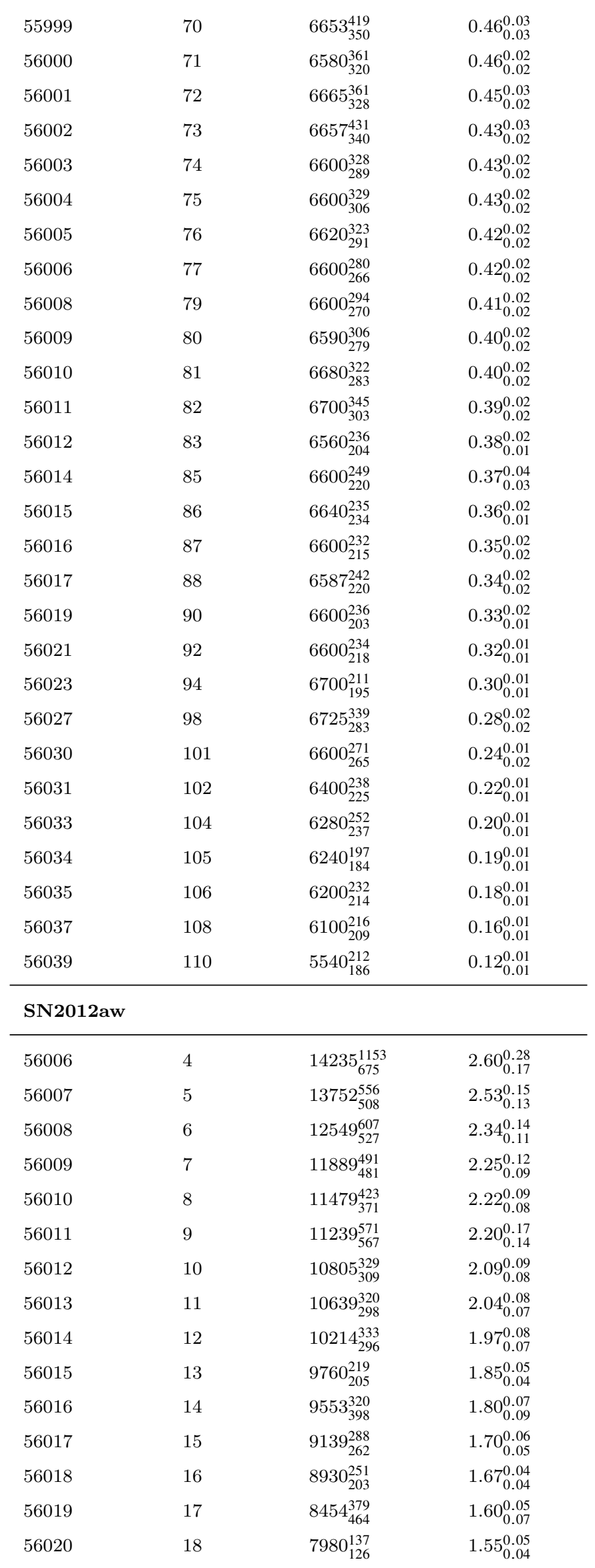


Faran et al.

Table A1 - continued A List of the Temperatures and Bolometric Luminosities

\begin{tabular}{|c|c|c|c|}
\hline 56022 & 20 & $7640_{111}^{124}$ & $1.49_{0.04}^{0.04}$ \\
\hline 56024 & 22 & $7400_{124}^{127}$ & $1.46_{0.04}^{0.04}$ \\
\hline 56025 & 23 & $7377_{226}^{182}$ & $1.46_{0.06}^{0.06}$ \\
\hline 56026 & 24 & $7260_{165}^{159}$ & $1.43_{0.05}^{0.06}$ \\
\hline 56027 & 25 & $7160_{150}^{160}$ & $1.40_{0.05}^{0.06}$ \\
\hline 56028 & 26 & $7120_{156}^{148}$ & $1.40_{0.05}^{0.06}$ \\
\hline 56029 & 27 & $7020_{144}^{162}$ & $1.37_{0.05}^{0.05}$ \\
\hline 56030 & 28 & $6965_{180}^{177}$ & $1.36_{0.05}^{0.05}$ \\
\hline 56031 & 29 & $6880_{148}^{146}$ & $1.34_{0.05}^{0.05}$ \\
\hline 56032 & 30 & $6792_{147}^{184}$ & $1.33_{0.05}^{0.05}$ \\
\hline 56033 & 31 & $6740_{141}^{136}$ & $1.32_{0.04}^{0.04}$ \\
\hline 56034 & 32 & $6700_{136}^{134}$ & $1.31_{0.04}^{0.04}$ \\
\hline 56035 & 33 & $6628_{143}^{176}$ & $1.30_{0.04}^{0.05}$ \\
\hline 56036 & 34 & $6621_{229}^{210}$ & $1.28_{0.05}^{0.05}$ \\
\hline 56037 & 35 & $6540_{208}^{207}$ & $1.27_{0.04}^{0.05}$ \\
\hline 56039 & 37 & $6440_{197}^{204}$ & $1.26_{0.04}^{0.05}$ \\
\hline 56040 & 38 & $6425_{196}^{212}$ & $1.27_{0.05}^{0.05}$ \\
\hline 56041 & 39 & $6438_{225}^{264}$ & $1.27_{0.05}^{0.05}$ \\
\hline 56042 & 40 & $6440_{189}^{218}$ & $1.28_{0.04}^{0.06}$ \\
\hline 56043 & 41 & $6380_{176}^{178}$ & $1.28_{0.05}^{0.05}$ \\
\hline 56044 & 42 & $6360_{173}^{179}$ & $1.28_{0.05}^{0.05}$ \\
\hline 56046 & 44 & $6280_{166}^{177}$ & $1.27_{0.05}^{0.05}$ \\
\hline 56047 & 45 & $6233_{172}^{183}$ & $1.26_{0.04}^{0.05}$ \\
\hline 56049 & 47 & $6160_{159}^{168}$ & $1.25_{0.05}^{0.05}$ \\
\hline 56050 & 48 & $6100_{138}^{128}$ & $1.26_{0.04}^{0.05}$ \\
\hline 56051 & 49 & $6000_{153}^{160}$ & $1.25_{0.04}^{0.05}$ \\
\hline 56052 & 50 & $5813_{147}^{165}$ & $1.24_{0.04}^{0.04}$ \\
\hline 56053 & 51 & $5780_{141}^{154}$ & $1.24_{0.04}^{0.04}$ \\
\hline 56054 & 52 & $6080_{209}^{209}$ & $1.27_{0.04}^{0.04}$ \\
\hline 56056 & 54 & $6140_{200}^{227}$ & $1.27_{0.04}^{0.04}$ \\
\hline 56057 & 55 & $6209_{238}^{263}$ & $1.29_{0.04}^{0.04}$ \\
\hline 56058 & 56 & $6280_{211}^{243}$ & $1.30_{0.04}^{0.04}$ \\
\hline 56059 & 57 & $6380_{222}^{253}$ & $1.31_{0.03}^{0.04}$ \\
\hline 56061 & 59 & $6420_{218}^{258}$ & $1.32_{0.03}^{0.04}$ \\
\hline 56062 & 60 & $6375_{238}^{249}$ & $1.31_{0.05}^{0.04}$ \\
\hline 56064 & 62 & $6420_{227}^{242}$ & $1.32_{0.04}^{0.05}$ \\
\hline 56066 & 64 & $6060_{161}^{166}$ & $1.29_{0.04}^{0.05}$ \\
\hline 56070 & 68 & $6000_{146}^{144}$ & $1.28_{0.04}^{0.05}$ \\
\hline 56072 & 70 & $5960_{152}^{164}$ & $1.26_{0.04}^{0.05}$ \\
\hline 56086 & 84 & $5900_{158}^{151}$ & $1.23_{0.05}^{0.05}$ \\
\hline 56087 & 85 & $5840_{140}^{154}$ & $1.21_{0.05}^{0.05}$ \\
\hline 56088 & 86 & $5480_{152}^{172}$ & $1.11_{0.05}^{0.06}$ \\
\hline 56089 & 87 & $5480_{139}^{156}$ & $1.11_{0.05}^{0.05}$ \\
\hline 56097 & 95 & $5760_{107}^{123}$ & $1.13_{0.05}^{0.05}$ \\
\hline
\end{tabular}


Table A1 - continued A List of the Temperatures and Bolometric Luminosities

\begin{tabular}{|c|c|c|c|}
\hline \multicolumn{4}{|c|}{ SN2012ec } \\
\hline 56154 & 11 & $10020_{326}^{345}$ & $2.26_{0.08}^{0.09}$ \\
\hline 56155 & 12 & $9780_{304}^{332}$ & $2.14_{0.07}^{0.08}$ \\
\hline 56158 & 15 & $9068_{534}^{926}$ & $1.85_{0.13}^{0.16}$ \\
\hline 56159 & 16 & $8080_{196}^{203}$ & $1.69_{0.04}^{0.04}$ \\
\hline 56165 & 22 & $6880_{107}^{101}$ & $1.44_{0.13}^{0.15}$ \\
\hline 56168 & 25 & $6640_{88}^{101}$ & $1.36_{0.12}^{0.14}$ \\
\hline 56171 & 28 & $6480_{100}^{92}$ & $1.33_{0.10}^{0.13}$ \\
\hline 56173 & 30 & $6620_{134}^{148}$ & $1.40_{0.19}^{0.29}$ \\
\hline 56176 & 33 & $6560_{142}^{136}$ & $1.40_{0.12}^{0.17}$ \\
\hline 56179 & 36 & $6200_{132}^{124}$ & $1.28_{0.09}^{0.11}$ \\
\hline 56181 & 38 & $6160_{122}^{115}$ & $1.27_{0.12}^{0.15}$ \\
\hline 56182 & 39 & $6120_{123}^{110}$ & $1.26_{0.05}^{0.06}$ \\
\hline 56186 & 43 & $6180_{129}^{125}$ & $1.30_{0.04}^{0.05}$ \\
\hline 56190 & 47 & $6120_{108}^{127}$ & $1.28_{0.05}^{0.05}$ \\
\hline 56195 & 52 & $6150_{136}^{152}$ & $1.32_{0.06}^{0.06}$ \\
\hline 56199 & 56 & $6220_{116}^{127}$ & $1.36_{0.04}^{0.04}$ \\
\hline 56202 & 59 & $6099_{151}^{159}$ & $1.32_{0.05}^{0.04}$ \\
\hline 56204 & 61 & $6000_{103}^{122}$ & $1.29_{0.04}^{0.04}$ \\
\hline 56208 & 65 & $5960_{122}^{133}$ & $1.30_{0.03}^{0.04}$ \\
\hline 56211 & 68 & $5840_{104}^{106}$ & $1.25_{0.03}^{0.04}$ \\
\hline 56212 & 69 & $5780_{122}^{107}$ & $1.24_{0.04}^{0.04}$ \\
\hline 56216 & 73 & $5680_{104}^{113}$ & $1.22_{0.05}^{0.05}$ \\
\hline \multicolumn{4}{|c|}{ SN2013ab } \\
\hline 56344 & 4 & $17620_{299}^{330}$ & $5.54_{0.16}^{0.18}$ \\
\hline 56345 & 5 & $15490_{534}^{596}$ & $4.73_{0.25}^{0.35}$ \\
\hline 56346 & 6 & $14258_{911}^{1073}$ & $4.15_{0.32}^{0.40}$ \\
\hline 56347 & 7 & $12768_{491}^{570}$ & $3.66_{0.16}^{0.19}$ \\
\hline 56348 & 8 & $11885_{254}^{268}$ & $3.35_{0.10}^{0.11}$ \\
\hline 56349 & 9 & $11210_{401}^{326}$ & $3.07_{0.15}^{0.13}$ \\
\hline 56350 & 10 & $10380_{112}^{105}$ & $2.73_{0.04}^{0.04}$ \\
\hline 56351 & 11 & $9996_{387}^{231}$ & $2.54_{0.12}^{0.09}$ \\
\hline 56352 & 12 & $9645_{266}^{264}$ & $2.41_{0.08}^{0.08}$ \\
\hline 56353 & 13 & $9349_{233}^{250}$ & $2.21_{0.09}^{0.10}$ \\
\hline 56354 & 14 & $9040_{160}^{183}$ & $2.09_{0.04}^{0.04}$ \\
\hline 56355 & 15 & $8928_{257}^{465}$ & $2.04_{0.06}^{0.13}$ \\
\hline 56356 & 16 & $9080_{284}^{282}$ & $2.04_{0.07}^{0.07}$ \\
\hline 56357 & 17 & $8826_{329}^{390}$ & $2.00_{0.07}^{0.09}$ \\
\hline 56358 & 18 & $8690_{257}^{267}$ & $1.96_{0.06}^{0.06}$ \\
\hline 56359 & 19 & $8620_{243}^{268}$ & $1.94_{0.05}^{0.06}$ \\
\hline 56360 & 20 & $8368_{286}^{315}$ & $1.87_{0.06}^{0.07}$ \\
\hline 56361 & 21 & $8220_{226}^{233}$ & $1.83_{0.04}^{0.05}$ \\
\hline 56362 & 22 & $7960_{201}^{229}$ & $1.75_{0.04}^{0.04}$ \\
\hline
\end{tabular}


Faran et al.

Table A1 - continued A List of the Temperatures and Bolometric Luminosities

\begin{tabular}{|c|c|c|c|}
\hline 56363 & 23 & $7920_{202}^{223}$ & $1.74_{0.04}^{0.04}$ \\
\hline 56364 & 24 & $7560_{182}^{202}$ & $1.67_{0.03}^{0.04}$ \\
\hline 56365 & 25 & $7540_{186}^{195}$ & $1.65_{0.03}^{0.03}$ \\
\hline 56366 & 26 & $7380_{198}^{215}$ & $1.57_{0.04}^{0.04}$ \\
\hline 56368 & 28 & $7190_{206}^{560}$ & $1.51_{0.04}^{0.10}$ \\
\hline 56370 & 30 & $7080_{335}^{354}$ & $1.44_{0.05}^{0.06}$ \\
\hline 56372 & 32 & $7016_{380}^{452}$ & $1.40_{0.05}^{0.08}$ \\
\hline 56373 & 33 & $6940_{309}^{349}$ & $1.37_{0.04}^{0.05}$ \\
\hline 56374 & 34 & $6800_{303}^{329}$ & $1.34_{0.04}^{0.05}$ \\
\hline 56376 & 36 & $6720_{290}^{327}$ & $1.31_{0.04}^{0.04}$ \\
\hline 56377 & 37 & $6680_{293}^{314}$ & $1.29_{0.04}^{0.04}$ \\
\hline 56378 & 38 & $6680_{309}^{333}$ & $1.28_{0.04}^{0.04}$ \\
\hline 56380 & 40 & $6620_{282}^{315}$ & $1.26_{0.03}^{0.04}$ \\
\hline 56381 & 41 & $6580_{275}^{315}$ & $1.25_{0.03}^{0.04}$ \\
\hline 56382 & 42 & $6500_{281}^{290}$ & $1.24_{0.03}^{0.04}$ \\
\hline 56383 & 43 & $6580_{276}^{313}$ & $1.22_{0.03}^{0.04}$ \\
\hline 56384 & 44 & $6650_{294}^{329}$ & $1.23_{0.03}^{0.04}$ \\
\hline 56388 & 48 & $6260_{255}^{278}$ & $1.19_{0.03}^{0.03}$ \\
\hline 56389 & 49 & $6220_{274}^{283}$ & $1.17_{0.03}^{0.03}$ \\
\hline 56390 & 50 & $6240_{267}^{293}$ & $1.15_{0.03}^{0.03}$ \\
\hline 56394 & 54 & $6299_{325}^{408}$ & $1.14_{0.03}^{0.05}$ \\
\hline 56395 & 55 & $6380_{271}^{319}$ & $1.15_{0.03}^{0.04}$ \\
\hline 56397 & 57 & $6500_{299}^{313}$ & $1.15_{0.03}^{0.04}$ \\
\hline 56398 & 58 & $6380_{284}^{303}$ & $1.13_{0.03}^{0.03}$ \\
\hline 56402 & 62 & $6300_{267}^{306}$ & $1.13_{0.03}^{0.03}$ \\
\hline 56403 & 63 & $6280_{264}^{307}$ & $1.10_{0.03}^{0.03}$ \\
\hline 56404 & 64 & $6380_{280}^{308}$ & $1.14_{0.03}^{0.03}$ \\
\hline 56405 & 65 & $6320_{283}^{292}$ & $1.13_{0.03}^{0.03}$ \\
\hline 56406 & 66 & $6240_{288}^{308}$ & $1.12_{0.03}^{0.03}$ \\
\hline 56407 & 67 & $6220_{264}^{293}$ & $1.11_{0.02}^{0.03}$ \\
\hline 56409 & 69 & $6347_{311}^{378}$ & $1.14_{0.03}^{0.04}$ \\
\hline 56411 & 71 & $6300_{278}^{293}$ & $1.13_{0.03}^{0.03}$ \\
\hline 56412 & 72 & $6220_{261}^{297}$ & $1.10_{0.03}^{0.03}$ \\
\hline 56413 & 73 & $6180_{251}^{302}$ & $1.07_{0.02}^{0.03}$ \\
\hline 56414 & 74 & $6120_{246}^{262}$ & $1.06_{0.02}^{0.02}$ \\
\hline 56415 & 75 & $6700_{344}^{381}$ & $1.13_{0.04}^{0.05}$ \\
\hline 56416 & 76 & $6460_{309}^{362}$ & $1.09_{0.03}^{0.04}$ \\
\hline 56419 & 79 & $6140_{252}^{286}$ & $1.05_{0.02}^{0.03}$ \\
\hline 56420 & 80 & $6080_{260}^{264}$ & $1.05_{0.02}^{0.02}$ \\
\hline 56421 & 81 & $5760_{224}^{244}$ & $1.01_{0.02}^{0.02}$ \\
\hline 56422 & 82 & $5960_{244}^{259}$ & $1.02_{0.02}^{0.02}$ \\
\hline 56423 & 83 & $5940_{237}^{264}$ & $1.01_{0.02}^{0.02}$ \\
\hline 56426 & 86 & $6115_{354}^{411}$ & $0.99_{0.03}^{0.04}$ \\
\hline 56427 & 87 & $5800_{236}^{251}$ & $0.95_{0.02}^{0.02}$ \\
\hline 56428 & 88 & $5760_{217}^{256}$ & $0.94_{0.02}^{0.02}$ \\
\hline
\end{tabular}


Table A1 - continued A List of the Temperatures and Bolometric Luminosities

\begin{tabular}{|c|c|c|c|}
\hline 56429 & 89 & $6020_{247}^{272}$ & $0.95_{0.02}^{0.02}$ \\
\hline 56430 & 90 & $5860_{223}^{255}$ & $0.92_{0.02}^{0.02}$ \\
\hline 56431 & 91 & $5760_{215}^{246}$ & $0.90_{0.02}^{0.02}$ \\
\hline 56432 & 92 & $6180_{280}^{330}$ & $0.90_{0.02}^{0.03}$ \\
\hline 56433 & 93 & $5776_{393}^{646}$ & $0.86_{0.03}^{0.06}$ \\
\hline 56434 & 94 & $5580_{207}^{229}$ & $0.83_{0.02}^{0.02}$ \\
\hline 56435 & 95 & $5663_{302}^{444}$ & $0.82_{0.02}^{0.02}$ \\
\hline 56436 & 96 & $5900_{254}^{299}$ & $0.81_{0.02}^{0.02}$ \\
\hline 56437 & 97 & $5780_{250}^{277}$ & $0.75_{0.02}^{0.02}$ \\
\hline 56438 & 98 & $5664_{317}^{365}$ & $0.69_{0.03}^{0.03}$ \\
\hline 56439 & 99 & $5760_{247}^{276}$ & $0.66_{0.01}^{0.01}$ \\
\hline 56440 & 100 & $5446_{287}^{318}$ & $0.62_{0.02}^{0.02}$ \\
\hline 56441 & 101 & $5480_{224}^{245}$ & $0.59_{0.01}^{0.01}$ \\
\hline 56442 & 102 & $5112_{477}^{460}$ & $0.54_{0.02}^{0.02}$ \\
\hline 56443 & 103 & $4860_{222}^{247}$ & $0.49_{0.02}^{0.02}$ \\
\hline 56444 & 104 & $4865_{353}^{470}$ & $0.46_{0.02}^{0.02}$ \\
\hline 56445 & 105 & $4840_{227}^{236}$ & $0.42_{0.02}^{0.02}$ \\
\hline 56446 & 106 & $4700_{204}^{233}$ & $0.40_{0.02}^{0.02}$ \\
\hline 56448 & 108 & $4380_{137}^{159}$ & $0.37_{0.01}^{0.01}$ \\
\hline 56449 & 109 & $4580_{148}^{176}$ & $0.35_{0.01}^{0.01}$ \\
\hline 56451 & 111 & $4500_{143}^{170}$ & $0.31_{0.01}^{0.01}$ \\
\hline 56454 & 114 & $4880_{234}^{237}$ & $0.27_{0.01}^{0.01}$ \\
\hline 56456 & 116 & $4620_{196}^{226}$ & $0.28_{0.01}^{0.01}$ \\
\hline 56457 & 117 & $4840_{181}^{180}$ & $0.27_{0.01}^{0.01}$ \\
\hline 56458 & 118 & $4880_{174}^{194}$ & $0.26_{0.01}^{0.01}$ \\
\hline 56460 & 120 & $4760_{176}^{173}$ & $0.25_{0.01}^{0.01}$ \\
\hline 56461 & 121 & $5520_{295}^{343}$ & $0.25_{0.01}^{0.01}$ \\
\hline 56464 & 124 & $4808_{208}^{243}$ & $0.24_{0.01}^{0.01}$ \\
\hline 56466 & 126 & $4770_{186}^{202}$ & $0.24_{0.01}^{0.01}$ \\
\hline 56468 & 128 & $4460_{193}^{209}$ & $0.26_{0.01}^{0.02}$ \\
\hline 56470 & 130 & $4620_{199}^{235}$ & $0.24_{0.01}^{0.01}$ \\
\hline 56476 & 136 & $4480_{187}^{220}$ & $0.24_{0.01}^{0.01}$ \\
\hline 56477 & 137 & $4800_{225}^{242}$ & $0.23_{0.01}^{0.01}$ \\
\hline 56478 & 138 & $4980_{248}^{256}$ & $0.21_{0.01}^{0.01}$ \\
\hline 56479 & 139 & $4680_{205}^{241}$ & $0.22_{0.01}^{0.01}$ \\
\hline 56480 & 140 & $4800_{225}^{242}$ & $0.21_{0.01}^{0.01}$ \\
\hline 56481 & 141 & $4640_{211}^{225}$ & $0.22_{0.01}^{0.01}$ \\
\hline 56485 & 145 & $5080_{248}^{278}$ & $0.20_{0.01}^{0.01}$ \\
\hline 56487 & 147 & $4940_{241}^{255}$ & $0.20_{0.01}^{0.01}$ \\
\hline 56489 & 149 & $4840_{250}^{287}$ & $0.19_{0.01}^{0.01}$ \\
\hline \multicolumn{4}{|c|}{ SN2013by } \\
\hline 56407 & 0 & $22890_{781}^{833}$ & $23.65_{1.62}^{1.80}$ \\
\hline 56408 & 1 & $19258_{704}^{1096}$ & $18.89_{1.74}^{2.62}$ \\
\hline 56409 & 2 & $16860_{512}^{549}$ & $16.23_{0.88}^{0.99}$ \\
\hline
\end{tabular}


Faran et al.

Table A1 - continued A List of the Temperatures and Bolometric Luminosities

\begin{tabular}{|c|c|c|c|}
\hline 56410 & 3 & $14923_{552}^{704}$ & $13.53_{0.79}^{1.09}$ \\
\hline 56411 & 4 & $13940_{343}^{350}$ & $12.41_{0.48}^{0.51}$ \\
\hline 56412 & 5 & $12422_{1173}^{491}$ & $10.76_{1.36}^{0.65}$ \\
\hline 56413 & 6 & $11304_{389}^{440}$ & $9.73_{0.43}^{0.51}$ \\
\hline 56414 & 7 & $10875_{477}^{562}$ & $9.09_{0.48}^{0.70}$ \\
\hline 56416 & 9 & $9991_{437}^{474}$ & $8.12_{0.32}^{0.34}$ \\
\hline 56417 & 10 & $9500_{275}^{289}$ & $7.47_{0.22}^{0.24}$ \\
\hline 56418 & 11 & $9340_{253}^{287}$ & $7.29_{0.19}^{0.23}$ \\
\hline 56419 & 12 & $8984_{309}^{487}$ & $6.88_{0.26}^{0.21}$ \\
\hline 56423 & 16 & $8272_{334}^{581}$ & $5.54_{0.19}^{0.35}$ \\
\hline 56424 & 17 & $8377_{359}^{379}$ & $5.36_{0.30}^{0.27}$ \\
\hline 56426 & 19 & $8060_{305}^{324}$ & $4.88_{0.14}^{0.16}$ \\
\hline 56427 & 20 & $7794_{607}^{736}$ & $4.58_{0.32}^{0.43}$ \\
\hline 56428 & 21 & $8455_{724}^{881}$ & $4.91_{0.43}^{0.63}$ \\
\hline 56429 & 22 & $8540_{743}^{895}$ & $4.89_{0.45}^{0.64}$ \\
\hline 56434 & 27 & $7180_{513}^{601}$ & $3.65_{0.18}^{0.25}$ \\
\hline 56436 & 29 & $7010_{486}^{587}$ & $3.48_{0.16}^{0.23}$ \\
\hline 56437 & 30 & $6880_{455}^{563}$ & $3.38_{0.14}^{0.20}$ \\
\hline 56438 & 31 & $7020_{485}^{574}$ & $3.38_{0.15}^{0.21}$ \\
\hline 56439 & 32 & $6720_{450}^{518}$ & $3.18_{0.12}^{0.16}$ \\
\hline 56440 & 33 & $6700_{436}^{520}$ & $3.18_{0.12}^{0.16}$ \\
\hline 56442 & 35 & $6620_{422}^{511}$ & $3.14_{0.11}^{0.15}$ \\
\hline 56444 & 37 & $6576_{430}^{493}$ & $3.12_{0.11}^{0.14}$ \\
\hline 56445 & 38 & $6640_{432}^{509}$ & $2.89_{0.10}^{0.14}$ \\
\hline 56447 & 40 & $6478_{518}^{602}$ & $2.88_{0.12}^{0.13}$ \\
\hline 56448 & 41 & $6720_{450}^{509}$ & $2.92_{0.11}^{0.15}$ \\
\hline 56449 & 42 & $6305_{635}^{807}$ & $2.79_{0.14}^{0.20}$ \\
\hline 56450 & 43 & $6180_{363}^{442}$ & $2.78_{0.08}^{0.09}$ \\
\hline 56451 & 44 & $6520_{417}^{484}$ & $2.81_{0.09}^{0.12}$ \\
\hline 56453 & 46 & $5900_{342}^{382}$ & $2.50_{0.07}^{0.07}$ \\
\hline 56456 & 49 & $5860_{332}^{384}$ & $2.50_{0.07}^{0.07}$ \\
\hline 56458 & 51 & $5760_{321}^{367}$ & $2.42_{0.06}^{0.07}$ \\
\hline 56460 & 53 & $5660_{302}^{363}$ & $2.35_{0.06}^{0.07}$ \\
\hline 56462 & 55 & $5720_{311}^{368}$ & $2.29_{0.06}^{0.06}$ \\
\hline 56464 & 57 & $5780_{321}^{375}$ & $2.23_{0.06}^{0.06}$ \\
\hline 56465 & 58 & $5620_{315}^{337}$ & $2.18_{0.06}^{0.06}$ \\
\hline 56466 & 59 & $5480_{294}^{324}$ & $2.15_{0.06}^{0.07}$ \\
\hline 56468 & 61 & $5820_{330}^{376}$ & $2.08_{0.06}^{0.06}$ \\
\hline 56470 & 63 & $5880_{341}^{377}$ & $2.03_{0.05}^{0.06}$ \\
\hline 56472 & 65 & $5620_{304}^{351}$ & $1.95_{0.05}^{0.06}$ \\
\hline 56473 & 66 & $5640_{309}^{348}$ & $1.89_{0.05}^{0.06}$ \\
\hline 56474 & 67 & $5660_{311}^{353}$ & $1.81_{0.05}^{0.05}$ \\
\hline 56476 & 69 & $5400_{281}^{319}$ & $1.70_{0.05}^{0.06}$ \\
\hline 56477 & 70 & $5420_{277}^{330}$ & $1.58_{0.05}^{0.05}$ \\
\hline 56478 & 71 & $5380_{280}^{315}$ & $1.54_{0.05}^{0.05}$ \\
\hline
\end{tabular}


Table A1 - continued A List of the Temperatures and Bolometric Luminosities

\begin{tabular}{|c|c|c|c|}
\hline 56481 & 74 & $5183_{278}^{296}$ & $1.40_{0.07}^{0.06}$ \\
\hline 56482 & 75 & $5173_{261}^{286}$ & $1.34_{0.05}^{0.05}$ \\
\hline 56483 & 76 & $5188_{258}^{294}$ & $1.26_{0.04}^{0.05}$ \\
\hline 56486 & 79 & $5080_{255}^{270}$ & $1.07_{0.04}^{0.05}$ \\
\hline 56487 & 80 & $5012_{263}^{282}$ & $1.00_{0.05}^{0.05}$ \\
\hline 56488 & 81 & $4816_{234}^{255}$ & $0.88_{0.04}^{0.05}$ \\
\hline 56489 & 82 & $5172_{433}^{391}$ & $0.76_{0.10}^{0.05}$ \\
\hline \multicolumn{4}{|c|}{ SN2013ej } \\
\hline 56500 & 3 & $13900_{841}^{724}$ & $4.00_{0.28}^{0.33}$ \\
\hline 56501 & 4 & $13053_{488}^{537}$ & $4.02_{0.24}^{0.29}$ \\
\hline 56502 & 5 & $12853_{665}^{749}$ & $4.33_{0.38}^{0.46}$ \\
\hline 56503 & 6 & $11493_{173}^{1170}$ & $4.14_{0.42}^{0.57}$ \\
\hline 56504 & 7 & $11488_{559}^{757}$ & $4.19_{0.34}^{0.40}$ \\
\hline 56505 & 8 & $11271_{407}^{646}$ & $4.08_{0.37}^{0.60}$ \\
\hline 56506 & 9 & $11145_{625}^{625}$ & $4.28_{0.29}^{0.36}$ \\
\hline 56507 & 10 & $10489_{450}^{534}$ & $4.07_{0.24}^{0.32}$ \\
\hline 56508 & 11 & $10452_{539}^{545}$ & $4.07_{0.26}^{0.31}$ \\
\hline 56509 & 12 & $10220_{433}^{480}$ & $4.02_{0.23}^{0.28}$ \\
\hline 56510 & 13 & $10018_{666}^{1224}$ & $3.92_{0.35}^{0.86}$ \\
\hline 56511 & 14 & $10600_{995}^{1302}$ & $4.41_{0.64}^{0.99}$ \\
\hline 56512 & 15 & $10560_{996}^{1288}$ & $4.43_{0.64}^{0.98}$ \\
\hline 56513 & 16 & $10339_{1121}^{1448}$ & $4.25_{0.74}^{1.16}$ \\
\hline 56514 & 17 & $10183_{972}^{1525}$ & $4.20_{0.59}^{1.12}$ \\
\hline 56515 & 18 & $9856_{1043}^{1277}$ & $3.91_{0.57}^{0.88}$ \\
\hline 56516 & 19 & $9410_{709}^{848}$ & $3.67_{0.38}^{0.51}$ \\
\hline 56520 & 23 & $9580_{1108}^{1516}$ & $3.83_{0.62}^{1.05}$ \\
\hline 56521 & 24 & $9784_{1125}^{1536}$ & $3.95_{0.69}^{1.13}$ \\
\hline 56522 & 25 & $9775_{1142}^{1558}$ & $3.84_{0.65}^{1.11}$ \\
\hline 56524 & 27 & $8975_{979}^{1547}$ & $3.22_{0.46}^{0.93}$ \\
\hline 56525 & 28 & $9054_{1187}^{1368}$ & $3.29_{0.56}^{0.82}$ \\
\hline 56526 & 29 & $8620_{855}^{1138}$ & $3.03_{0.38}^{0.61}$ \\
\hline 56528 & 31 & $8640_{860}^{1144}$ & $2.93_{0.37}^{0.59}$ \\
\hline 56529 & 32 & $8737_{896}^{1154}$ & $2.93_{0.40}^{0.60}$ \\
\hline 56533 & 36 & $8180_{791}^{1006}$ & $2.50_{0.28}^{0.43}$ \\
\hline 56534 & 37 & $8060_{781}^{1004}$ & $2.42_{0.26}^{0.41}$ \\
\hline 56538 & 41 & $7040_{962}^{1359}$ & $1.91_{0.21}^{0.43}$ \\
\hline 56539 & 42 & $7560_{1000}^{1394}$ & $2.02_{0.27}^{0.51}$ \\
\hline 56541 & 44 & $7520_{997}^{1363}$ & $1.92_{0.25}^{0.47}$ \\
\hline 56544 & 47 & $7400_{971}^{1301}$ & $1.84_{0.23}^{0.42}$ \\
\hline 56546 & 49 & $6920_{840}^{1157}$ & $1.65_{0.16}^{0.31}$ \\
\hline 56553 & 56 & $6520_{757}^{968}$ & $1.48_{0.12}^{0.21}$ \\
\hline 56554 & 57 & $6940_{853}^{1124}$ & $1.53_{0.16}^{0.28}$ \\
\hline 56560 & 63 & $6580_{751}^{1023}$ & $1.35_{0.11}^{0.21}$ \\
\hline 56562 & 65 & $6520_{757}^{968}$ & $1.35_{0.11}^{0.19}$ \\
\hline
\end{tabular}


42 Faran et al.

Table A1 - continued A List of the Temperatures and Bolometric Luminosities

\begin{tabular}{llll}
56563 & 66 & $6800_{805}^{1097}$ & $1.34_{0.12}^{0.24}$ \\
56567 & 70 & $6440_{732}^{953}$ & $1.25_{0.09}^{0.17}$ \\
56568 & 71 & $6340_{713}^{915}$ & $1.21_{0.09}^{0.16}$ \\
56573 & 76 & $6600_{771}^{1003}$ & $1.16_{0.10}^{0.18}$ \\
56574 & 77 & $6800_{805}^{1097}$ & $1.16_{0.11}^{0.21}$ \\
56577 & 80 & $6920_{843}^{1125}$ & $1.14_{0.11}^{0.21}$ \\
56578 & 81 & $6540_{758}^{981}$ & $1.05_{0.09}^{0.15}$ \\
56589 & 92 & $6700_{784}^{1056}$ & $0.79_{0.07}^{0.13}$ \\
56594 & 97 & $6560_{759}^{993}$ & $0.59_{0.05}^{0.09}$ \\
56596 & 99 & $5980_{630}^{812}$ & $0.44_{0.02}^{0.04}$ \\
56600 & 103 & $6420_{837}^{1135}$ & $0.22_{0.02}^{0.04}$ \\
56607 & 110 & $6180_{933}^{1358}$ & $0.15_{0.01}^{0.03}$ \\
56610 & 113 & $6980_{1539}^{2924}$ & $0.16_{0.02}^{0.09}$ \\
56613 & 116 & $5960_{1111}^{1815}$ & $0.12_{0.01}^{0.03}$ \\
56616 & 119 & $6440_{1072}^{1638}$ & $0.13_{0.01}^{0.03}$ \\
56617 & 120 & $5960_{1075}^{1722}$ & $0.13_{0.01}^{0.03}$ \\
\hline
\end{tabular}

